\title{
Abstracts of the 9th Workshop Probiotics and Prebiotics Spanish Society of Probiotics and Prebiotics (SEPyP)
}

Zaragoza, Spain, February 15-16, 2018

\section{Abstracts}

Guest Editors

Ascensión Marcos, Research Professor at the Spanish National Research Council, Madrid Guillermo Alvarez Calatayud, President of the Spanish Society of Probiotics and Prebiotics (SEPyP), Madrid 
S. Karger

Medical and Scientific Publishers Basel · Freiburg · Paris · London . New York · Chennai · New Delhi Bangkok $\cdot$ Beijing $\cdot$ Shanghai $\cdot$ Tokyo . Kuala Lumpur · Singapore · Sydney
Disclaimer

The statements, opinions and data contained in this publication are solely those of the individual authors and contributors and not of the publisher and the editor(s). The appearance of advertisements in the journal is not a warranty, endorsement, or approval of the products or services advertised or of their effectiveness, quality or safety. The publisher and the editor(s) disclaim responsibility for any injury to persons or property resulting from any ideas, methods, instructions or products referred to in the content or advertisements.

Drug Dosage

The authors and the publisher have exerted every effort to ensure that drug selection and dosage set forth in this text are in accord with current recommendations and practice at the time of publication. However, in view of ongoing research, changes in government regulations, and the constant flow of information relating to drug therapy and drug reactions, the reader is urged to check the package insert for each drug for any change urged to check the package insert for each drug for any change
in indications and dosage and for added warnings and precautions. This is particularly important when the recommended agent is a new and/or infrequently employed drug.
All rights reserved.

No part of this publication may be translated into other languages, reproduced or utilized in any form or by any means, electronic or mechanical, including photocopying, recording, microcopying, or by any information storage and retrieval system, without permission in writing from the publisher or in the case of photocopying, direct payment of a specified fee to the Copyright Clearance Center (see 'General Information').

(c) Copyright 2018 by S. Karger AG,

P.O. Box, $\mathrm{CH}-4009$ Basel (Switzerland)

e-ISBN 978-3-318-03059-4

\section{KARGER}

E-Mail karger@karger.com

www.karger.com/anm 


\section{Workshop of the Spanish Society of Probiotics and Prebiotics. SEPyP- 2018.}

Modulation of gut microbiota by using probiotics, prebiotics and synbiotics is acquiring a great interest in order to treat several diseases, mainly regarding gastrointestinal diseases, such as different types of diarrhoea (infectious, antibiotic-associated diarrhoea, traveler's diarrhoea, lactose intolerance, etc.), functional disorders (infant colic or irritable bowel syndrome) or inflammatory processes (ulcerative colitis). In addition, they are being successfully used in different women's diseases (vulvovaginitis and mastitis) and their effects on allergies such as atopic dermatitis and on infections prevention (from the premature neonate to the elderly people) have been positively evaluated in different studies.

Concurrently to the numerous research studies that have appeared during the last few years dedicated to increase the knowledge of the native microbiota, the research on animal models and humans open up new fields to future applications with the supplementation of these organisms and bioactive compounds. There are studies that could support its use in nutritional disorders (obesity, malnutrition), neurological and behavioural disorders (autism, depression, anxiety), periodontal disease and the possible prevention of several types of cancer. Finally, progress is being made on the effects that dysbiosis (unbalanced, poor or distorted microbiota) may cause on the appearance of cardiovascular diseases, arteriosclerosis, diabetes, hypercholesterolemia, metabolic syndrome, etc.

The Spanish Society of Probiotics and Prebiotics (Sociedad Española de Probióticos y Prebióticos, SEPyP) is a scientific non-profit organization founded in 2010 , devoted to the development and promotion of the scientific knowledge and research, as well as the clinical applications of probiotics and prebiotics on the diverse microbiota from different regions of the body. SEPyP is a multidisciplinary Forum where all the health professionals who belong to this Society (around one thousand) come from different disciplines: physicians, pharmacists, veterinarians, microbiologists, basic researchers, immunologists, nutritionists, midwives, etc. SEPyP is also supported by the most important companies in this area of expertise.

Since the first year SEPyP was founded, a workshop has been annually organized with the purpose of promoting the scientific evidence about the role and possible benefits of probiotics and prebiotics among health professionals, in addition of being a unique forum for the exchange of the most relevant research advances on the field of the autochthonous microbiota. This year this workshop is held on the 15th and 16th February in Zaragoza (Spain).

The scientific programme of this workshop (SEPyP-2018) is included in this special supplement of 'Annals of Nutrition and Metabolism', that includes 2 round tables, 4 extraordinary conferences, 7 workshops, and 73 abstracts ( 20 oral communications plus 53 posters). All these presentations are dealing with key topics, such as microbiota and lactation or the microbiota-gut-brain axis, and the development and knowledge of new probiotics and their role in different areas of the organism to tackle diverse diseases and microbiota disorders.

To sum up, we want to express our gratitude to the participating companies for their invaluable help and, very specially, to the organizing and scientific committees, as well as to the speakers and debate chair persons at the round tables and workshops that are making possible this meeting one more year.

Ascensión Marcos Guillermo Alvarez Calatayud Editors 



\section{Dear friends:}

On behalf of the Board of Directors of the Spanish Society of Probiotics and Prebiotics (SEPyP), I would like to welcome you to the IX Workshop on Probiotics, Prebiotics and Health: Scientific Evidence to be held in Zaragoza on the 15th and 16th of February 2018.

SEPyP is a non-profit scientific organization devoted to the promotion and dissemination of scientific knowledge, research, clinical application of the diverse regions of microbiota of the organism, as well as the impact of probiotics and prebiotics on health. SEPyP has currently more than 900 scientific members and is supported by the main companies in this sector.

As in previous editions, and following the interdisciplinary framework that characterizes our scientific association, our guest speaker spots will be filled with various national and international experts who will address many topics of interest that will undoubtedly be the focus of debate among attendees. These may participate in various workshops that will cover a broad range of the main health areas. In this meeting near one hundred of new communications from both the field of basic research and its clinical applicability is included.

The meeting will be held in the Auditorium - Palacio de Congresos, emblematic building of the city of Zaragoza, and more than ideal location both from an educational and cultural point of view. The excellent coordination of the meeting is led by $\mathrm{M}^{\mathrm{a}}$ Lourdes De Torres Aured working at the Miguel Servet Hospital, who counts with extensive experience in the organization of this type of events. On behalf of the Board of Directors of SEPyP, we also want to express our special thanks to the generosity of the participants and local administrations.

Without any further ado, hoping to meet a very attractive programme, I invite you to participate in our Workshop that has become almost a yearly tradition for many health professionals (physicians, pharmacists, veterinarians, basic researchers, nurses, dieticians, etc.) whom little by little, are getting closer to the exciting world of microbiota, probiotics and prebiotics.

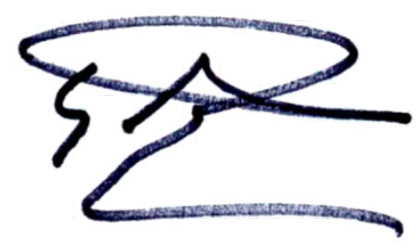

Guillermo Álvarez Calatayud SEPyP's President 



\section{Organizing Committee's President Introduction Letter}

Hosting friends and colleagues in Zaragoza is always a joy and an honour.

When the reason to be your hostess in my City, is to have received from the Board of Directors of SEPyP the order to organize the IX Workshop, that honour is loaded with fearful responsibility.

Responsibility because the Scientific Programme that has been prepared is of such a high level that the local organization must obligatorily live up to it. However, I think that within this beautiful city, and its competent and impacting infrastructure as a venue, it will not be difficult for us to meet the best frame to achieve a successful event.

When you arrive in Zaragoza, you arrive to a city of the five Cultures, because Iberians, Romans, Muslims, Jews and Christians have passed through here; and each of them have left their mark on recognizable and distinguished monuments throughout the world. Nevertheless, above all these populations have left their mark on the cordiality of the people of Zaragoza and their openness of minds.

The city of Zaragoza is cosy and quiet because its people have an open and generous nature that make any stranger feel at home. This warm atmosphere comes together with its rich and varied cuisine, which you can enjoy seated from any restaurant's orderly and exquisite menu, or standing with excellent "tapas" that make you stop being measured and rational.

Standing or sitting you will taste the fried breadcrumbs with anything ("con todo"), the traditional ternasco in the baker's oven or in the thousand ways that the innovative chef is presenting, the borage with potato and AOVE (Extra Virgin Olive Oil) or with rice and clams, the "chilindrón" style chicken, the famous "lobster from the Ebro river", or the ranch of "cucharada y paso atrás"...

Having three original wine varieties (Cariñena, Borja, Calatayud) and one original variety of olive oil called "Sierra del Moncayo", also helps a lot to be able to show off with friends and visitors, while being offered some fruits of Aragon and maraschino cherries, or some "cobblestones" together with their "stones of the river."

Zaragoza is also called a congress city because, given its geographical location, it is at the center of multiple interprovincial connections by road or rail. Additionally, because we count with the luxury of having large venues where many visitors can be located at the same time, venues with state-of-the-art technology that make speakers and assistants comfortable in congresses.

Zaragoza as the capital of Aragon is the main headquarters of two universities (UNIZAR and USJ) and their respective Research and Study Centers, which places it at the forefront of research relating to food technology and health. Serving as an example of research, technology and health, we have the only independent Brewer who has put on the market a beer (which tastes like beer) but without gluten and alcohol in it that has helped celiac people so much.

Having said all the above, it is quite true that the task of organizing the IX Workshop of SEPyP has not been a very complicated task for this hostess, because as we say, when you have good wicks, you get good baskets. Soon enough, you will have solid first-hand proof about all of the references I have mentioned.

For all these reasons, I would like to thank the SEPyP board of directors with all my heart for deciding that Zaragoza can be an ad hoc city with the Excellence of the Society and that as coordinator I would be able to meet their expectations.

I would like to greet you personally, because smiling at friends and receiving them, are the highest pleasures for any self-respecting professional.

I look forward to seeing you in Zaragoza!!!

Welcome to Zaragoza!

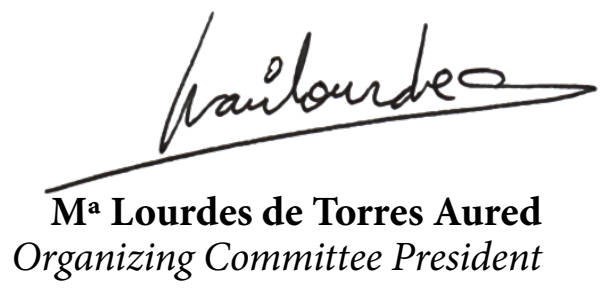





\section{Committees}

\section{Organizing and Scientific Committees}

Organizing Committee President:

$M^{a}$ Lourdes de Torres Aured

Functional Unit of Dietetics and Nutrition. Miguel Servet University Hospital. Zaragoza.

President of the Scientific Committee:

Guillermo Álvarez Calatayud

Department of Pediatrics. Gregorio Marañón Hospital. Madrid.

Members of Organizing and Scientific Committees

$M^{a}$ Lourdes de Torres Aured

Functional Unit of Dietetics and Nutrition. Miguel Servet University Hospital. Zaragoza.

Guillermo Álvarez Calatayud

Department of Pediatrics. Gregorio Marañón Hospital. Madrid.

Francisco Guarner Aguilar

Departament of Gastroenterology. Hospital Vall d'Hebron. Barcelona.

Ascensión Marcos Sánchez

Institute of Science and Technology of Food and Nutrition ICTAN-CSIC. Madrid.

Teresa Requena Rolanía

Food Sciences Research Institute, CIAL (CSIC- UAM). Madrid.

Mónica de la Fuente del Rey

Biological Sciences Department. Universidad Complutense de Madrid.

Jose Manuel Martín Villa

School of Medicine. Universidad Complutense de Madrid.

Gaspar Pérez Martínez

Institute of Agrochemistry and Food Technology, IATA-CSIC. Valencia.

Juan Evaristo Suárez Fernández

Oviedo University. Asturias.

Juan Miguel Rodríguez Gómez

Veterinary School. Universidad Complutense de Madrid.

Alfonso Clemente Gimeno

Experimental Station of Zaidín (EEZ-CSIC). Granada.

Abelardo Margolles Barro

Institute of Dairy Products. IPLA-CSIC. Oviedo.

Fernando Azpiroz Vidaur

Departament of Gastroenterology. Hospital Vall d'Hebron. Barcelona.

\section{Industrial Advisory Council Members}

क) AB.BIOTICS
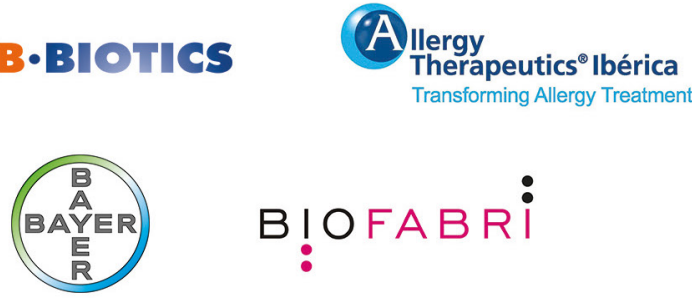

B!OFABRi

\section{BIOSEARCH}

$L$ I F E

CASEN RECORDATI

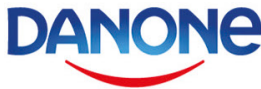

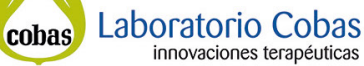

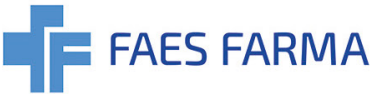

FARMASIERRA

LABORATORIOS CALIDADE INNOVACIÓN

\section{FERRING \\ PRODUCTOS FARMACÉUTICOS \\ GRIFOLS}

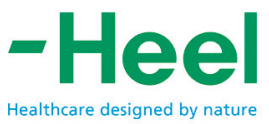

GRUPO ITALFARMACO

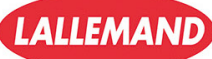

LALLEMAND HEALTH SOLUTIONS

NUTRICIA

Early Life Nutrition

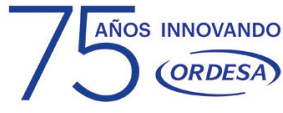

\section{Pileje}

Schwabe Farma Ibérica

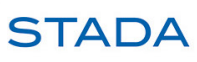

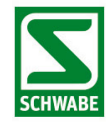

Iberica
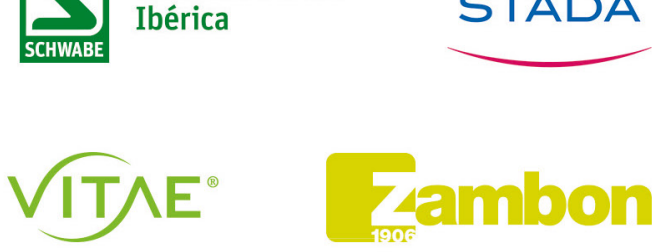



\section{THURSDAY, 15TH FEBRUARY}

$9.00-9.30 \mathrm{~h}$.

Welcome and Opening.

Room Luis Galve

$9.30-10.30 \mathrm{~h}$.

ROUND TABLE I: Microbiota and Lactation.

Room Luis Galve

Chairs:

Luis Peña Quintana

Gastroenterology and Pediatrics Nutrition Unit at the Maternity Hospital of Las Palmas. Health Sciences Faculty. University of Las Palmas de Gran Canaria. Gran Canaria. Spain.

Jorge Amil

Pediatrics Department. São João Hospital. Oporto. Portugal.

Microbiota and diet at the mother-child binomial.

Speaker:

Carmen Collado Amores

Agricultural Chemistry and Food Technology Institute (IATA-CSIC). Valencia. Spain.

Probiotics, prebiotics and synbiotics in infant formulas. Speaker:

José Manuel Moreno Villares

Pediatrics Division. University of Navarra Clinic. Madrid. Spain.

$10.30-11.30 \mathrm{~h}$.

ROUND TABLE II: Microbiota-Gut-Brain Axis.

Room Luis Galve

Chairs:

Francisco Guarner

Researcher at the Gastroenterology Unit. University Hospital Vall d'Hebron. Barcelona. Spain.

Angel Lanas

Head of Digestive System Service of University Clinical Hospital of Zaragoza. Scientific Director of Health Research Institute of Aragón.

\section{Evidence for a microbiota-brain axis.}

Speaker:

Premysl Bercik

Medicine. Division of Gastroenterology. McMaster University. Hamilton. Canada.

\section{Probiotics in IBS.}

Speaker:

Eamonn Quigley

Doctor. Division of Gastroenterology. Houston Methodist Hospital. Houston. EEUU.
11.30 - $12.30 \mathrm{~h}$.

\section{Coffee Break and Poster Session.}

Hall

Chairs:

Luz Taboada

Pediatrician. Hospital San Rafael. Madrid. Spain.

Jimena Pérez Moreno

Gastroenterology and Nutrition Division. General University Hospital Gregorio Marañón of Madrid. Spanish Society of Probiotics and Prebiotics (Sociedad Española de Probióticos y Prebióticos, SEPyP). Spain.

Claudia Herrera de Guise

Research Institute of Vall d'Hebron. Barcelona. Doctor specializing in Gastroenterology at the Crohn-Colitis care unit. Researcher at the Institute of the Hospital Vall d'Hebron. Barcelona.

\section{$12.30-14.30 \mathrm{~h}$.}

\section{Session: Clinical Uses.}

Room Luis Galve

Chairs:

Vicente Varea Calderón

Gastroenterology Pediatrician. Barcelona. Spain.

Eduardo Bajador Andreu

Chief, Division of Gastroenterology. Assistant Professor at the Medicine Department. University Hospital Miguel Servet. University of Zaragoza.

\section{Microbiota and Celiac Disease.}

Speaker:

Elena Verdú

Associate Professor. Division of Gastroenterology. Farncombe Institute. McMaster University. Canada.

\section{Oral Presentations}

$14.30-16.30 \mathrm{~h}$.

Lunch Break.

$16.30-18.30 \mathrm{~h}$.

Workshop: Clinical Uses. Adults.

Room Luis Galve

Coordinator:

Francisco Guarner

Researcher at the Gastroenterology Unit. University Hospital Vall d'Hebron. Barcelona. Spain.

Speakers:

Juan José Sebastián Domingo

Chief, Division of Gastroenterology. Hospital Royo Villanova. Adjunct Professor at the Medicine Department. University of Zaragoza.

Rafael Tojo González

Division of Gastroenterology. University Hospital of Cabueñes. Gijon. Spain. 
$16.30-18.30 \mathrm{~h}$.

Workshop: Clinical Uses II. Paediatrics.

Room Mariano Gracia

Coordinators:

Beatriz Espín

Doctor specializing in Pediatrics. Division of Gastroenterology, Hepatology and Pediatrics Nutrition. University Children's Hospital Virgen del Rocío. Sevilla.

Juan José Diaz

Division of Gastroenterology. Hospital of Oviedo. Spain.

Debate Session: Probiotics in Formulas for Healthy Infants. For:

Gerardo Rodríguez

University Hospital Lozano Blesa. Zaragoza.

Against:

Marta Suarez

Division of Neonatology. University Hospital of Asturias. Oviedo.

Presentation: Probiotics in the Inflammatory Bowel Disease.

Speaker:

Vicente Varea

Senior of the Division of Pediatric Gastroenterology at the Hospital San Juan de Dios of Barcelona.

\section{$16.30-18.30 \mathrm{~h}$.}

Workshop: Nutrition and Dietetics.

Hall Dr. Muñoz (Hospital U. Miguel Servet)

Coordinator:

$M^{a}$ Rosaura Leis Trabazo

Chief, Division of Pediatric Nutrition. University Hospital of Santiago de Compostela.

Dysbiosis and disease: Rational uses of probiotics, prebiotics and synbiotics.

Speaker:

$M^{a}$ Rosaura Leis Trabazo

Chief, Division of Pediatric Nutrition. University Hospital of Santiago de Compostela.

Microbiota alteration and obesity, cause or consequence?

Speaker:

Javier Acha Pérez

Chief, Division of Endocrinology and Nutrition. University Hospital

Miguel Servet. Zaragoza.

\section{Current diet and lifestyle and gut microbiota}

Speaker:

Diana Boj Carceller

Endocrinologist in the University Hospital Lozano Blesa, Zaragoza.

Effects of the oncologist treatments on the microbiota alteration.

Speaker:

Marta Villarino Sanz

Dietician-Nutritionist. Hospital Infanta Sofía. San Sebastián de los Reyes. Madrid.
$16.30-18.30 \mathrm{~h}$.

Workshop: Nursing/Midwifes.

Hall H. I. (University Hospital Miguel Servet)

Coordinator:

Carmen Martín Salinas

Professor of Nutrition at the Bachelor of Nursing. Autonomous University of Madrid.

Obesity control in the expectant woman: The satiating effect of the yoghurt and the fiber as a sign of a healthy diet. Case studies.

Speaker:

Carmen Martín Salinas

Professor of Nutrition at the Bachelor of Nursing. Autonomous University of Madrid.

Promotion of the microbiota as an improvement of the lactation and the prevention of mastitis. Case studies.

Speaker:

$M^{a}$ Ángeles Checa

Midwife. Healthcare Center Arrabal. Zaragoza. Spain.

Varied diet and fermented milk intake in the education of food groups during childhood and adolescence. Case studies.

Speaker:

Marina Francés Pinilla

Pediatric Nurse of Primary Healthcare. Zaragoza.

Lactose Intolerance vs malabsorption or maldigestion: Yoghurt and other fermented milk products intake. Case studies.

Speaker:

Mari Lourdes de Torres Aured

Functional Unit of Dietetics and Nutrition. University Hospital Miguel Servet. Zaragoza. Spain.

\subsection{0 - $18.30 \mathrm{~h}$.}

Workshop: Veterinary Sciences.

Room 7

Coordinators:

Juan Miguel Rodríguez

Faculty of Veterinary Medicine. Complutense University of Madrid. Spain.

Teresa Requena Rolanía

Food Science research institute (CIAL: CSIC- UAM). Madrid. Spain.

Microbiota and Probiotics in Aquaculture.

Speaker:

Tania Pérez Sánchez

Faculty of Veterinary Medicine. University of Zaragoza.

Microbiota y probiotics in porcine livestock.

Speaker:

Susana Martín Orúe

Animal Nutrition and Welfare Service (SNiBA). Department of Animal and Food Science. Autonomous University of Barcelona (Universitat

Autònoma de Barcelona, UAB). 
Microbiota and probiotics in Veterinary science in the context of the action plan on antimicrobial resistance.

Speaker:

Odón Julián Sobrino Abuja

Spanish Ministry of Agriculture and Fisheries, Food and Environment.

$16.30-18.30 \mathrm{~h}$.

Workshop: Omics.

Room 11

Coordinator:

Abelardo Margolles

Institute of Dairy Products of Asturias (IPLA-CSIC). Spain.

Omics approaches for the study of the oral microbiome. Speaker:

Alex Mira

Director of the laboratory of Human Microbiome in the Genomic Area and Health of the foundation FISABIO. Valencia. Spain.

Metabolomics: a fundamental tool in the study of the metabolism and the microbiota.

Speaker:

Xavier Correig

Department of Electronic Engineering. Rovira i Virgili University. CIBERDEM. Tarragona. Spain.

Genetics of host-microbiota interactions: relevance to gastrointestinal disease.

Speaker:

Mauro D'Amato

Professor of the medical research institute BioDonostia. Gipuzkoa. Spain.

16.30 - $18.30 \mathrm{~h}$.

Workshop: Pharmacology.

Lecture hall 4 (University Hospital Miguel Servet)

Coordinator:

Ana Ma Mateos Lardiés

Member of the Governing Board and Coordinator of the Nutrition and Gastroenterology Group of the SEFAC Association.

Presentation of the SEFAC-SEPyP standards of practice and the consensus document on the use of preparations with probiotics and/or prebiotics in the community pharmacy.

Speakers:

Guillermo Álvarez-Calatayud

Pediatrics Department. Hospital Gregorio Marañón. Madrid. Spain.

Ana Ma Mateos Lardiés

Member of the Governing Board and Coordinator of the Nutrition and Gastroenterology Group of the SEFAC Association.

Advice of Preparations with Probiotics in the Community Pharmacy. CLINICAL CASE STUDIES.

Speakers:

Ana Ma Mateos Lardiés

Member of the Governing Board and Coordinator of the Nutrition and Gastroenterology Group of the SEFAC Association.

Ana Rodríguez-Sampedro

Member of the Nutrition and Gastroenterology Group of the SEFAC Association. SEFAC Development Area.
18.30 - $19.00 \mathrm{~h}$.

Coffee Break and Posters Session.

Hall

19:00-20:00 h.

General Meeting.

Room Luis Galve

\section{FRIDAY, 16TH FEBRUARY}

\section{$8.30-10.30 h$.}

\section{Session: Immunonutrition.}

Room Luis Galve

Chairs:

Ascensión Marcos

Research Professor at the Institute of Food Science, Technology and Nutrition (ICTAN). Spanish National Research Council (CSIC).

Mónica de la Fuente

Complutense University of Madrid. Spain.

Novel Probiotics (I): Akkermansia muciniphila.

Speaker:

Amandine Everard

Metabolism and Nutrition research group (MNut). Louvain Drug Research Institute (LDRI) Catholic University of Louvain (UCL).

Oral Presentations.

$10.30-11.30 \mathrm{~h}$.

\section{Coffee Break and Posters Session.}

Hall

Chairs:

Gaspar Pérez Martínez

Agricultural Chemistry and Food Technology Institute (IATA-CSIC).

Valencia. Spain.

José Manuel Martín Villa

Microbiology Department. Complutense University of Madrid. Spain. Miguel Gueimonde Fernández

Dairy Microbiology and Biochemistry Department. Dairy Research Institute of Asturias (IPLA). Spanish National Research Council (CSIC). Asturias.

11:30-14:00 h.

Session: Microbiology and Veterinary Science. Room Luis Galve

Coordinators:

Abelardo Margolles

Dairy Research Institute of Asturias (IPLA-CSIC.) Spain.

Agustín Ariño Moneva

Professor at University of Zaragoza. Division of Nutrition and Food

Science. Spain. 


\section{Novel Probiotics (II): Faecalibacterium prausnitzii}

Speaker:

Rebeca Martín

French National Institute for Agriculture Research. INRA. Paris.

\section{Oral Presentations}

\section{$14.00 \mathrm{~h}$.}

\section{Closing Lecture: "Prebiotic Effect of Beer"}

Room Luis Galve

\section{Chair:}

Mari Lourdes de Torres Aured

Functional Unit of Dietetics and Nutrition. University Hospital Miguel

Servet. Zaragoza. Spain.

Speaker:

Antonio Fumanal Sopena

Chemist. Master Brewer of AMBAR. Zaragoza. Spain.

Chair:

Guillermo Álvarez Calatayud

Pediatrics Department. Hospital Gregorio Marañón. Madrid. Spain.

\section{Awards Ceremony}

SEPyP Honorary Member Recognition

\section{Workshop Closing}

\section{Certification}

Granted the recognition of professional interest (Reconocimiento de Interés Sanitario - RIS) by the under secretary of Health, Social Services and Equality of Madrid.

Certification of on-site activities (Acreditación de Actividades Presenciales) requested to the Committee of Continuing Education of Health Professions of Aragón. 


\section{THE AUTOCHTHONOUS MICROBIOTA AND PROBIOTICS: EFFICACIOUS COMPLEMENTS IN THE FIGHT AGAINST INFECTIONS AND THE RIS- ING OF ANTIBIOTIC RESISTANCE}

\author{
(1) J.M. Rodríguez, ${ }^{(2)}$ F. Guarner, ${ }^{(3)}$ G. Álvarez Calatayud., ${ }^{(4)}$ E. Suárez. \\ (1)Department of Nutrition and Food Science, Complutense \\ University of Madrid, Spain; (2)Digestive System Research \\ Unit, University Hospital Vall d'Hebron, Barcelona, Spain; \\ ${ }^{(3)}$ Department of Gastroenterology and Infant Nutrition, \\ University Hospital Gregorio Marañón, Madrid, Spain; \\ ${ }^{(4)}$ Department of Functional Biology, University of Oviedo, Spain.
}

\author{
Corresponding Authors: \\ Juan M. Rodríguez, PhD \\ Professor \\ Department of Nutrition and Food Science, Complutense \\ University of Madrid, Avda. Puerta de Hierro s/n., (2)(8)(0)(4)(0) \\ Madrid, Spain \\ E-Mail jmrodrig@ucm.es \\ J. Evaristo Suárez, PhD \\ Professor \\ Department of Functional Biology, University of Oviedo, Spain, \\ Campus El Cristo s/n., 33006 Oviedo, Asturias, Spain \\ E-Mail evaristo@uniovi.es
}

\section{ABSTRACT}

Antibiotics are the cornerstone on which anti-infectious therapy is based. Their use has provoked the selection of resistant pathogens, which are progressively hindering treatment success. In addition, antibiotic pressure on the autochthonous microbiota alters the homeostasis of the composing communities, attenuate their beneficial effects and make difficult the treatment of endogenous infections. Therefore, it is convenient to design strategies that complement antibiotherapy, the utilization of probiotic microorganisms being among the most promising ones. Probiotics have demonstrated therapeutic effects on digestive (lactose intolerance, diarrheas of different etiologies, pouchitis), urogenital (vaginitis, vaginosis, uncomplicated cystitis) and mammary (mastitis) processes. Moreover, they reduce the frequency of recurrences due to their multiplication at the mucosal surfaces and present synergistic effects with antibiotics when rationally used. Their use should lead to diminishing antibiotic usage, to attenuation of the pressure in favour of resistant microorganisms and to their substitution by susceptible populations that, in general, present a higher fitness.

Key Words: autochthonous microbiota / probiotics / infections /antibiotics / antibiotic resistance

\section{THE AUTOCHTHONOUS MICROBIOTA}

The autochthonous microbiota is constituted by the microbial communities that colonize, in a rather stable manner, the epidermal surface and all the host's ducts and cavities that communicate with the outside. The microbiota is essential for human and animal development and for protection against infection, to the point that its presence is usually perceived only when predisposing circumstances convert some of its components into opportunistic pathogens $[1,2]$. A paradigmatic example could be the propionibacteria that colonize the epidermis. These microorganisms degrade serum triglycerides, releasing fatty acids that, together with the propionic acid generated from their metabolism of carbohydrates, decrease the skin $\mathrm{pH}$ and create inhospitable conditions for the establishment of pathogens. However, the excess of fat release that occurs during puberty can turn them into acne-causing agents [3].

Given that the prevailing conditions in the colonized surfaces are different, each will harbour a characteristic and well-adapted microbiota that tends to remain stable. Therefore, there is usually a greater similarity between the microbiotas that colonize the same cavity of different individuals than between the distinct ones of the same person. Thus, in the vagina there is an absolute predominance of bacteria of the genus Lactobacillus during the fertile period of women, while the colon microbiota is extraordinarily diverse, including prokaryotic (bacteria and archaea) and eukaryotic (molds, yeasts, protozoa and helminths) organisms, and also viruses, which may infect both the microbiota and our own cells [2].

\section{FUNCTIONS OF THE INDIGENOUS MICROBIOTA}

The beneficial effects of the autochthonous microbiota can be grouped into two broad categories: metabolic complementation and protection against infections.

\section{Metabolism complementation}

The microbiota contributes to anabolism through the supply of essential nutrients, such as vitamins and some amino acids and fatty acids, therefore reducing the amounts that must be incorporated through the diet. They are also essential for the digestion of complex carbohydrates, which they transform into short chain fatty acids; these promote peristalsis and are essential nutritional sources for colonocytes; in fact, it is estimated that more than $10 \%$ of the energy provided by the diet arises from the catabolism of polysaccharides by the colonic microbiota [4].

\section{Protection against infections}

This function is accomplished through two complementary means: a) microbial antagonism, which hinders the settlement of potentially pathogenic microorganisms on our mucosal surfaces and is based on three main mechanisms: interference with colonization, production of antimicrobial compounds and co-aggregation with pathogens; and b) collaboration to the correct differentiation and maturation of the immune system. 


\section{Interference with colonization}

The microbiota members display adhesins on their surface that specifically recognize receptors linked to the outside of epithelial cells, which are also used by pathogens. Therefore, harm microorganisms will not be able to attach to the mucosa and will be dragged out by the fluids that bathe its surface [5]. Additionally, the microbiota is adapted to the conditions of the cavities it colonizes (available nutrients, $\mathrm{pH}, \mathrm{pO} 2$, etc.), so that the establishment of pathogens will only occur when the autochthonous microbiota has been altered (e.g., after treatment with antibiotics) or there is an extraordinarily large invasion (e.g., cholera or toxiinfections caused by Salmonella spp.).

\section{Production of antimicrobial compounds}

The components of the microbiota generate a wide array of substances with antimicrobial capacity. First, most members of our microbiota are strict anaerobic or aerotolerant organisms that produce organic acids from carbohydrate fermentation. The resulting low $\mathrm{pH}$ is microbicidal for most pathogens, which are adapted to the neutral or slightly alkaline conditions of our internal milieu. Hydrogen peroxide is another antimicrobial compound that is mainly produced by the vaginal lactobacilli. Finally, many bacteria synthesize antimicrobial peptides called bacteriocins, which form pores in the bacterial membranes and/or inhibit the synthesis of the bacterial wall $[6,7]$.

\section{Coaggregation with pathogens}

Some members of the microbiota tend to aggregate, so that they trap invading organisms in their lumps and contribute to prevent their attachment to the mucosa; moreover, the proximity between the autochthonous and the invading organisms increases the effectiveness of the antimicrobial compounds that might be synthesized by the former [8].

\section{Induction of immune system differentiation}

During fetal development, macrophages, epithelial, dendritic and some other cell types, develop receptors that will recognize surface microbial polymers, such as the bacterial peptidoglycan and lipopolysaccharides or the cell wall components of fungi. Along the perinatal period, superficial components of the first microbial colonizers are detected by epithelial, M, and dendritic cells, which migrate to the lymphoid primordia, Peyer's patches and mesenteric nodules, contributing to their maturation, the recognition of the exposed antigens by the resident $\mathrm{B}$ and $\mathrm{T}$ cells, and the initiation of IgA secretion by plasma cells. In contrast, the capacity of IgA synthesis by axenic ("germ-free") animals is approximately 50 times lower than that of conventionally-bred ones; in addition, they present numerous abnormalities in their lymphoid structures, including hypoplastic Peyer's patches and a large reduction in the number of lymphoid follicles [9]. Furthermore, the tight junctions between enterocytes are strengthened upon interaction with surface microbial polymers, while division of these cells is also stimulated, thus increasing the depth of crypts and promoting the differentiation of the cells located to their bottom into Paneth cells, which produce a variety of eukaryotic antimicrobial peptides (defensins, cathelicidins, angiopoietin 4, etc.). The mucosa-microbe interactions also increase the number of goblet cells and the subsequent secretion of mucus and, as a result, the protection of the epithelium against direct interaction with luminal microorganisms [10].

\section{ANTIBIOTICS AND MICROBIOTA}

Antibiotics inhibit essential metabolic processes for the development of microorganisms, while respecting our own metabolism. They are key elements of the preventive and therapeutic arsenal against infections, which also includes general sanitation measures (habitat sanitation, availability of drinkable water, sewage treatment, puerperal and food hygiene, etc.) and the development of vaccines and immunosera.

Globally, these measures have led to drastic reductions in the prevalence of morbidity and mortality, due to infectious diseases, in countries with advanced health. Thus, for example, in 1900 the most common illnesses were respiratory infections, tuberculosis and gastroenteritis, with rates close to 200 deaths per 100,000 inhabitants, in each case. However, coronary heart disease and cancer had taken the lead at the beginning of this century, with only respiratory infections remaining (in sixth place) among the ten most prevalent causes of death [11]. This reduction in the incidence of infections has led to a considerable increase in life expectancy, from less than 35 years during the XIX century (in part linked to a very high infant mortality) to more than 80 years at present [12].

However, the application of antibiotic therapy has led to the selection of pathogenic organisms that have become resistant, thus making chemotherapy ineffective. Our microbiota suffers the same selective pressure from antibiotics, although this effect has remained largely ignored until recently. The consequences of this fact are two:

a) A decrease in microbial diversity, which entails the attenuation of the beneficial effects it produces and, probably, the appearance or exacerbation of autoimmune and metabolic syndrome-associated processes.

b) The acquisition of gene determinants of resistance that have transformed some components of the microbiota in difficult-to-treat opportunistic pathogens and, possibly, in dispersers of those genes.

\section{Reduction of biodiversity}

Antibiotics exert a deep impact on the microbiota. Oral administration of ciprofloxacin ( $500 \mathrm{mg} / 12 \mathrm{~h}$ for 5 days) in adult volunteers halved the number of species detectable by $16 \mathrm{~S}$ rRNA gene sequencing [13]. Diversity was restored one or two weeks after the end of treatment, but some species never recovered $[13,14]$. This problem is especially worrying in children living in developed countries who, on average, receive around three antibiotic treatments before reaching the age of three [15]. Their consumption in the infant population represents $\sim 25 \%$ of the total human use and the younger the children, the greater the utilization of antibiotics. Unfortunately, in some cases they are inappropriately prescribed at these ages (mainly for viral infections that do not improve with antibiotics). A stable intestinal microbial community is not established until children are 2-3-years-old and antibiotics might provoke underrepresentation of some key taxonomic groups [16]. Consequently, excessive growth of other microorganisms (e.g., those intrinsically resistant to the antibiotic used) may occur; therefore, there is a loss of microbial diversity in the affected mucosal surface, although the total number of colonizing organisms may remain unaffected or even increase [17]. This may have two consequences; on one hand, it might negatively affect the functions exerted by the susceptible species and, on the other, the rising of new pathologies, such as antibiotic-associated diarrhoeas of diverse etiologies and pseudomembranous colitis, a severe condition mainly due to the overgrowth of Clostridium difficile.

9th Workshop SEPyP 
Similarly, the systemic use of antibiotics may affect the vaginal lactobacilli, leading to bacterial vaginosis or vaginal candidiasis. In many cases, these conditions are recurrent since the causal organisms (Gardnerella vaginalis, Candida spp.) are indigenous to the vagina and, therefore, are well adapted to its conditions, thus making difficult to fight them and restore the previous Lactobacillus dominance. Mucosal dysbiosis may be associated to other pathologies, although it is not clear whether the microbiota alteration is the cause or the consequence of the condition. Thus, people affected by problems as diverse as the metabolic syndrome (fatty liver, type 2 diabetes, dyslipidemia, obesity, cardiovascular risk), inflammatory bowel diseases (Crohn's disease, ulcerative colitis, necrotizing enterocolitis), irritable bowel syndrome, allergies (atopic eczema, asthma, food intolerance), alcoholic hepatitis and complications of cirrhosis, colorectal cancer, behavioural disorders within the spectrum of autism, etc., usually present a significant decrease in the diversity of their intestinal microbiota [18]. It is, therefore, conceivable that the disturbances produced by antibiotics, especially during the early stages of life, combined with individual genetic susceptibility and other factors, might have a lasting impact on the microbiota-host homeostasis that leads to disease or disease predisposition.

Selection of antibiotic-resistant components of the microbiota

The microbiota is permanently settled on our mucosal surfaces and, thus, is exposed to all antibiotic treatments taken by the host. The resulting selective pressure is far more intense than what pathogens must stand because they will only be confronted to it during symptomatic infection. Therefore, certain members of the microbiota that may behave as opportunistic pathogens and originate endogenous infections, have acquired resistance genes that make their treatment difficult. Notable examples of this are Staphylococcus epidermidis, a permanent colonizer of the skin and some mucosal surfaces, and enterococci, whose main habitat is the large intestine $[19,20]$. In addition, in cases where the resistance is due to inactivation or enzymatic modification of the drug, satellite phenomena may appear, especially in cavities colonized by complex microbiotas, such as the colon.

The situation is aggravated because many of the gene determinants of resistance are located in self-transmissible or mobilizable vectors, such as plasmids and conjugative transposons [21, 22]; this converts the microbiota into a reservoir of these genes and, probably, into vehicles for dispersing them to pathogens during infectious processes.

In conclusion, it is well established that the autochthonous microbiota exerts evident beneficial effects to the host and that its antibiotic driven alteration causes the attenuation of those advantages and converts its members into reservoirs of antibiotic resistance traits. Therefore, it seems that restoring the microbiota would be a rational initiative to recover our mutualistic relationship. This is, in fact, the philosophy underlying the probiotic concept.

\section{PROBIOTIC ORGANISMS: AN EFFECTIVE COM- PLEMENT IN THE FIGHT AGAINST INFECTION AND ANTIBIOTIC RESISTANCE}

Probiotics are defined as "live microorganisms that, when administered in adequate amounts, confer a benefit to the health of the host" $[23,24]$. Some properties of probiotic organisms are charac-

9th Workshop SEPyP teristic of the taxonomic group to which they belong (for example, the production of lactic acid by lactobacilli) while other properties are held only by some strains (co-aggregation with certain pathogenic species, prevention of diarrhea by rotavirus, neuromodulation ...) [24]. On the other hand, the probiotic effect depends on the doses (typically $>108$ cells/dose) and the route of administration; thus, the oral route implies that, if the target is the colon, the organism in question must support the microbicidal effect of gastric acidity, bile and the digestive enzymes of the pancreas and the small intestine.

The use of probiotics is not intended to replace the autochthonous microbiota, but rather to prevent or combat infections derived from the existence of predisposing circumstances that facilitate pathogen settlement. For this purpose, preparations of harmless microorganisms that have the GRAS (Generally Regarded As Safe) or QPS (Qualified Presumption of Safety) qualifications granted by the FDA and the EFSA, respectively, are used. Typically, they belong to one of the taxonomic groups that colonize the concerned cavity. In this way, competitiveness against pathogens and contribution to the restoration of the normal physicochemical conditions of that cavity are ensured. This will facilitate the recovery of the native microbiota and the return to normality. Moreover, probiotic organisms are very well characterized, usually their complete genome and the proteins it encodes are known, to ensure that they do not harbor virulence factors or transmissible antibiotic resistance determinants.

It appears to be convenient to define here the prebiotic and synbiotic concepts, which are related to that of probiotic and whose homophony could lead to confusion. Prebiotics are "food ingredients that, when selectively fermented, produce specific changes in the composition and/or activity of the gastrointestinal microbiota, conferring benefits on the host's health" [25, 26, www.sepyp. es/pdf/docConsensoPrebioticos.pdf). Synbiotics are mixtures of one or more probiotic organisms with one or more prebiotic compounds that exert a synergistic beneficial effect (http://www.sepyp. es/es/wiki).

In recent years, the research on probiotics has experienced a great boom, achieving scientific and clinical advances that are allowing the development and commercialization of preparations with proven therapeutic activity. In parallel, the population is becoming increasingly aware of the close relationship between the autochthonous microbiota and health, so that the demand for probiotics is growing rapidly. Unfortunately, some companies have taken advantage of this situation to apply the term "probiotic" to products that do not fit into this concept and/or whose presumed benefits lack any scientific or clinical basis. This misuse has generated some skepticism in part of the health care community. This is one of the reasons why a commission of experts convened jointly by FAO and WHO in 2001, recognized the need to establish guidelines for the evaluation of the efficacy and safety of probiotics [23]. In 2002, a mixed working group of both institutions developed these guidelines, which included the requirements to be fulfilled to grant a product the probiotic consideration [27]. The concept of probiotic has been updated with the publication of consensus documents launched by the Spanish Society of Probiotics and Prebiotics (SEPyP) (http://www.sepyp.es) [28], the International Scientific Association for Probiotics and Prebiotics (ISAPP) [24] and the World Gastroenterology Organization (WGO) http://www. 
worldgastroenterology.org/guidelines/global-guidelines/probiotics-and-prebiotics). The last one provides lists of organisms with indication of the processes on which they may be beneficial, the level of evidence of the recommendation and the references that support their use.

The process of qualifying a microorganism to be used in the prevention or therapy of a pathological process begins with the selection of potentially useful strains and concludes with the presentation of the pharmaceutical form in a format that guarantees the viability of the organisms. The process is not simple, and it has to contemplate various aspects (scientific, clinical, technological, legal, economic, communicative ...) that, although sometimes are not easy to conjugate, can and must be compatible with each other [29]. Overall, the greatest evidence of benefits associated with the administration of probiotic preparations is focused on the following pathologies [30]: (1) lactose intolerance; (2) acute and antibiotic-associated diarrheas; (3) gut inflammatory processes, such as ulcerative colitis or pouchitis; (4) vaginal and lower urinary tract infections; and (5) lactational mastitis. Other conditions for which treatment with probiotics present promising data include infant colic, irritable bowel syndrome, functional abdominal distension, necrotizing enterocolitis, and eradication of Helicobacter pylori. Their effects on allergies and on the prevention of infections (from the premature newborn to the elderly) has also been evaluated, although the existing data still require well-designed clinical studies and enough number of volunteers to confirm or disprove the expectations created so far.

\section{Lactose intolerance}

Both lactobacilli and bifidobacteria produce lactase, which cleaves lactose into glucose and galactose, and makes it assimilable by the small intestine. This prevents the disaccharide from reaching the large intestine undigested and the typical symptoms of intolerance: flatulence, abdominal distension, and diarrhoea. This property has been approved as a health claim by the EFSA [31], based on thirteen human studies in which evident and plausible beneficial effects were demonstrated after the administration of yoghurt to intolerant individuals (target population).

\section{Acute infectious and antibiotic-associated diarrhoeas}

The working groups of the European Society of Gastroenterology, Hepatology and Pediatric Nutrition (ESPGHAN) and the WGO have communicated, based on many studies and meta-analyses, that there is enough clinical evidence on the benefits of certain probiotic strains in the prevention and treatment of acute gastroenteritis (especially those caused by rotavirus and norovirus) and diarrhoeas associated to antibiotic therapy [32, $33,34,35,36]$. The probiotics would occupy the mucosa damaged by viruses (in the first case) while they would protect the epithelium against colonization by opportunistic pathogens (in the second), constituting an emergency solution that would attenuate the symptoms and facilitate recolonization by the indigenous microbiota.

\section{Pouchitis}

The artificial rectum (pouch) generated in patients who have suffered a colostomy becomes infected frequently. One of the most effective methods of healing is oral administration of probiotics which, in addition to helping in elimination of the symptoms, prevents the recurrences that are often observed after antibiotic treatment $[37,38]$.
Infectious pathology of the vagina and lower urinary tract

The treatment of vaginosis and vaginitis usually involves organic acids, antibiotics or probiotic lactobacilli. The first two strategies are frequently followed by relapses. The use of lactobacilli results in a reduction of this effect because its therapeutic action is prolonged since they are living organisms that multiply on the mucosa. Instilled lactobacilli regenerate the acidic conditions that antagonize pathogens and provide the appropriate environment for recolonization by the indigenous lactobacilli $[2,22,39]$.

Urinary tract infections are due, mainly, to the colonization of the urethra by enteric microorganisms and are more frequent in women than in men because of obvious anatomical reasons. The vagina can act as a barrier against the migration of enteric bacteria due to its acidic $\mathrm{pH}$. However, the decrease in resident lactobacilli that accompanies vaginal pathology and that is also observed in most postmenopausal women, eliminates this protective effect and, consequently, increases the likelihood of urethral infection. Therefore, treatment with probiotic lactobacilli (accompanied by intravaginal estrogen therapy in postmenopausal women) will cause a decrease of vaginal $\mathrm{pH}$ and have positive effects on this pathology [40, 41].

In this context, the conclusions of the first consensus document of the Spanish Association for the Study of Menopause (AEEM) on the use of probiotics in Gynecology [42] were the following: 1. The indigenous microbiota plays a relevant role in the prevention of vaginal infections. 2. Probiotics are effective as adjuvants in the treatment of the most frequent vaginal infections. 3. Probiotics improve the cure rates of vaginal diseases, reduce the recurrence of bacterial vaginosis, decrease the recurrence of urinary tract infections, and improve cure rates of vulvovaginitis caused by Candida spp.

\section{Lactational mastitis}

Human milk contains its own microbiota [43], which may become altered by using antibiotics during the perinatal period [44]. This would favour the colonization of the gland by antibiotic-resistant staphylococci, streptococci and corynebacteria, which may induce mastitis. Some strains of lactobacilli isolated from human milk have proved to be a good option for the adjuvant treatment of acute and subacute mastitis and on their prevention in women who suffered from this mammary pathology after previous pregnancies [45-47].

\section{NEXT GENERATION PROBIOTICS}

Most of the current probiotic microorganisms are lactobacilli and bifidobacteria. This is because they are the only components of the microbiota whose safety is universally recognized (as already commented, they are considered GRAS and/or QPS organisms). In fact, they are essential for the manufacture of fermented foods and, consequently, they are consumed in enormous quantities without having been associated with pathological effects. For that reason, their physiology is very well known, which facilitates their propagation and storage and makes possible the realization of probiotic aptitude studies. However, an enormous effort of isolation, characterization, safety assessment and probiotic ability with many other components of the indigenous microbiota is being carried out, so it is foreseeable that the catalog of microbial species with probiotic strains will increase significantly in the future. The correlation be-

9th Workshop SEPyP 
tween the presence of bacteria of the genus Barnesiella in the colon and the resistance to invasion by vancomycin-resistant enterococci [48], the antagonistic effect of secondary bile salts produced by Clostridium scindens on the proliferation of Clostridium difficile [49] and the beneficial role of Faecalibacterium prausnitzii on large intestine homeostasis [50] are good examples.

\section{FECAL AND VAGINAL TRANSFER}

The transfer of complete communities that could potentially restore complex ecosystems is a complementary strategy to the use of singular probiotic organisms. Fecal and vaginal transfers are the most advanced of these procedures.

Fecal transplantation consists in the administration of a stool suspension, obtained from a healthy person, to another one with an infectious digestive pathology through enteral feeding tubes, colonoscopy or enema. In this way, the entire intestinal microbiota is transferred, including microorganisms that currently cannot be propagated and/or preserved and, consequently, cannot be administered as conventional probiotics. Faecal transplants have shown extraordinary efficacy in the treatment of Clostridium difficile infections, including the almost total elimination of recurrences, which are the main drawbacks of antibiotic treatments for pseudomembranous colitis [51-53].

Vaginal transfer is based on the fact that intestinal colonization of newborns begins with the ingestion of microorganisms present in the genital tract and the perineal region of the mother. Therefore, children born by Caesarean section are not colonized adequately and this may have undesirable consequences, not only in relation to the proper acquisition of the microbial community, but also in the adequate maturation of the immune system. For this reason, sterile gauzes introduced into the vagina (and, therefore, impregnated with the microorganisms present there) of mothers with Caesarean section-scheduled deliveries were used recently to rub their neonates' gums, face and body immediately after birth. Their colonization patterns were more like those observed in neonates born by vaginal delivery than to those delivered by Caesarean section [54].

Despite their empirical usefulness, faecal and vaginal transplantation face two important practical problems: (a) the extraordinary complexity and variability of these biological samples, which makes standardization impossible; and (b) their possible contamination with pathogens that might asymptomatically colonize the healthy donor but that could cause pathology in the recipient, usually elderly people with underlying conditions that may weaken their immune system. Additionally, the feces might contain toxic or even carcinogenic metabolites. Therefore, it is necessary to develop approaches that allow the transfer of microbial communities in a reproducible and safe way. An example of this is the minimal microbiota concept that is described below.

\section{MINIMAL OR SYNTHETIC MICROBIOTA}

Minimal or synthetic microbiotas are complex mixtures of microorganisms obtained from a given ecosystem, propagated as pure cultures, and mixed in known proportions before being instilled in an organic cavity (usually the colon). In this way, the microbial variability inherent to faecal samples and the possible presence of

9th Workshop SEPyP pathogens or toxic metabolites are eliminated. The best example of minimal microbiota is the so-called repopulate, elaborated from the pure cultures of 33 bacterial strains isolated from faeces of a single healthy donor. This complex mixture has been used to treat cases of recurrent infection by $C$. difficile in which the antibiotic therapy had failed, and the results were very promising [55].

\section{POTENTIAL OF PROBIOTICS IN THE FIGHT AGAINST ANTIBIOTIC RESISTANCE}

Antibiotic therapy almost inevitably leads to the selection of resistant microorganisms. There is a direct relationship between the rates of resistance and the frequency of use of chemotherapeutic agents, although other factors, such as inadequate prescription, over-the-counter use and low adherence to treatments, also have a decisive influence [15]. Therefore, any strategy aimed to complementing and thus reduce the use of antibiotics should lower the rate of resistant organisms' emergence.

Among the antibiotic use rationalization strategies, probiotics should play a prominent role because, as indicated above, they exert therapeutic effects in a series of digestive and genitourinary infections and reduce recurrences due to their multiplication on the mucosal surfaces. The combined use of antibiotics and probiotics must take into account that they are live microorganisms while the former inhibit key routes of the microbial metabolism. Thus, if the probiotic is susceptible to the action of a given antibiotic, the respective doses should be intercalated and spaced as much as possible or even, administration of the probiotic should wait until antibiotherapy has been completed. On the other hand, there are cases in which the treatments can be combined; for example, the antifungals used for the treatment of candidiasis do not affect bacteria and, conversely, the use of an antibacterial agent could be combined with the administration of Saccharomyces boulardii; similarly, glycopeptides, such as teicoplanin and vancomycin, do not usually inhibit the growth of lactobacilli, and the same happens with clindamycin and metronidazole, two antibiotics that are frequently used in the treatment of anaerobic infections.

In conclusion, although antibiotics continue to be irreplaceable for the treatment of infectious diseases, their complementation with other strategies, such as the use of bacteriophages, bacteriocins and, above all, probiotics, can lead to the rationalization of their consumption and, thus, to the decrease of resistant organisms' selection pressure.

\section{LINES OF ACTION AND RESEARCH PROPOSED BY THE SPANISH SOCIETY FOR PROBIOTICS AND PREBIOTICS (SEPYP) WITHIN THE FRAME- WORK OF THE SPANISH NATIONAL PLAN FOR ANTIBIOTIC RESISTANCE}

A better knowledge of the human microbiota may provide valuable tools to reduce the impact of antibiotic-resistant bacteria emergence, one of the major challenges for Public Health at present and, probably, in the future. The Spanish Society for Probiotics and Prebiotics (SEPyP) proposes the following lines of action and research in this field: 
(a) Evaluation of the oral, gastrointestinal and genitourinary microbiota of women throughout pregnancy in a search for biomarkers associated to the etiopathogenesis of preterm birth, the influence of the microbiota composition on the outcome and the impact of anti-infectious interventions on the prevalence of antibiotic resistant organisms. Design of intervention microbial mixes aimed to restore healthy microbiotas that may contribute to lower prematurity incidence.

(b) Study of newborn gastrointestinal microbiota colonization (at term and premature) to identify biomarkers associated with the risk of infection, sepsis or necrotizing enterocolitis, as well as the influence of the antimicrobial treatments on the microbiota composition and evolution, with emphasis on possible long-term undesirable effects. Devise microbial mixtures aimed to be used successively and in parallel with diet changes to promote healthy colonization of children.

(c) Analysis of the skin, nasopharyngeal, respiratory, biliary and other overlooked microbiotas to define biomarkers of healthy colonization in those locations. Proposal of probiotic preparations that might improve the outcome of infections affecting such locations.

(d) Testing the microbiota evolution in its different locations along the life to formulate interventional strategies (nutritional, microbiota reposition, modulation of the immune system) aimed to counteract changes that might increase the predisposition to suffer pathological conditions and the subsequent use of antibiotics for their treatment.

(e) Assessment of the possible synergistic effects arising from the combined use of antibiotics and probiotics/prebiotics/synbiotics in the treatment of infectious diseases and on the reduction of recurrences, which might allow lowering of antibiotic usage and, with it, of iatrogenic effects and selection of resistant microorganisms.

(f) Search for conditions that may allow individual propagation of fastidious, major microbiota members. Devise of microbial mixtures that may mimic the composition and functions of the microbial communities that colonize the different body surfaces/cavities to allow substitution of uncharacterized probiotic preparations (faecal, vaginal) by defined formulations to minimize their drawbacks. Determination of the compatibility of these synthetic microbiotas with chemotherapy to define synergistic, neutral or antagonistic effects.

(g) Based on the above, promote actualization of the National and European Regulations for probiotic/prebiotic/synbiotic treatments, elaboration of prescription lists by EMEA and inclusion in the financed lists of medicaments by the Public Health Authorities.

\section{REFERENCES:}

1. Brüssow H: Microbiota and the human nature: know thyself. Environ Microbiol 2015; 17: 10-15.

2. Martín R, Escobedo S, Martín C, Suárez JE: Microbiota autóctona: funciones. Microbioma humano. In: Probióticos, probióticos y salud: Evidencia científica. A. Calatayud G, Marcos A, Margolles A (Eds.), pp. 1-10. Madrid, Ergón, 2016.

3. Chen YE, Tsao H: The skin microbiome: current perspectives and future challenges. J Am Acad Dermatol 2013; 69: 143-155.

4. Koropatkin NM, Cameron EA, Martens EC: How glycan metabolism shapes the human gut microbiota. Nat Rev Microbiol 2012; 10: $323-335$
5. Martín R, Martín C, Escobedo S, Suárez JE, Quirós LM: Surface glycosaminoglycans mediate adherence between HeLa cells and Lactobacillus salivarius Lv72. BMC Microbiol 2013; 13: 210-221.

6. Turner DL, Brennan L, Meyer HE, Lohaus C, Siethoff C, Costa $H$, González B, Santos H, Suárez JE: Solution structure of plantaricin C, a novel lantibiotic. Eur J Biochem 1999; 264: 833-839.

7. Martin R, Suárez JE: Biosynthesis and degradation of $\mathrm{H} 2 \mathrm{O} 2$ by vaginal lactobacilli. Appl Environ Microbiol 2010; 76: 400-405.

8. Boris S, Suárez JE, Vázquez F, Barbes C: Adherence of human vaginal lactobacilli to epithelial vaginal cells. Infect Immun 1998; 66: 1985-1989.

9. Sekirov I, Russell SL, Antunes LC, Finlay BB: Gut microbiota in health and disease. Physiol Rev 2010; 90: 859-904.

10. Maynard CL, Elson CO, Hatton RD, Weaver CT: Reciprocal interactions of the intestinal microbiota and immune system. Nature 2012; 489: 231-241.

11. Madigan MT, Martinko JM, Dunlap PV, Clark DP: Biología de los Microorganismos. Madrid, Pearson, 2009.

12. Burns H: Germ theory: invisible killers revealed. BMJ 2007; 334 Suppl 1: s11.

13. Dethlefsen L, Huse S, Sogin ML, Relman DA: The pervasive effects of an antibiotic on the human gut microbiota, as revealed by deep 16S rRNA sequencing. PLoS Biol. 2008; 6: e280.

14. Dethlefsen L, Relman DA: Incomplete recovery and individualized responses of the human distal gut microbiota to repeated antibiotic perturbation. Proc Natl Acad Sci U S A. 2011; 108 Suppl 1: 4554-4561.

15. Hicks LA, Taylor TH Jr, Hunkler RJ: More on U.S. outpatient antibiotic prescribing, 2010. N Engl J Med 2013; 369: 1175-1176.

16. Yassour $M$, Vatanen $T$, Siljander $H$, Hämäläinen AM, Härkönen T, Ryhänen SJ, et al: Natural history of the infant gut microbiome and impact of antibiotic treatment on bacterial strain diversity and stability. Sci Transl Med 2016; 8: $343 \mathrm{ra81.}$

17. Panda S, El khader I, Casellas F, Guarner F, Manichanh C: Short-term effect of antibiotics on human gut microbiota. PLoS One 2014; 9: e95476.

18. Holmes E, Li JV, Marchesi JR, Nicholson JK: Gut microbiota composition and activity in relation to host metabolic phenotype and disease risk. Cell Metab 2012 7; 16: 559-564.

19. Ubeda C, Taur Y, Jenq RR, Equinda MJ, Son T, Samstein M, et al: Vancomycin-resistant Enterococcus domination of intestinal microbiota is enabled by antibiotic treatment in mice and precedes bloodstream invasion in humans. J Clin Invest. 2010; 120: 4332-4341.

20. Cercenado E: Epidemiología de la infección por Gram positivos resistentes. Rev Esp Quimioter 2016; 29 Suppl 1: 6-9.

21. Ammor MS, Gueimonde M, Danielsen M, Zagorec M, van Hoek AH, de los Reyes-Gavilán CG, et al: Two different tetracycline resistance mechanisms, plasmid-carried tet $(L)$ and chromosomally located transposon-associated tet $(M)$, coexist in Lactobacillus sakei Rits 9. Appl Environ Microbiol 2008; 74: 1394-1401.

22. Martín R, Soberón N, Vázquez F, Suárez JE: La microbiota vaginal: composición, papel protector, patología asociada y perspectivas terapéuticas. Enferm Infecc Microbiol Clin 2008; 26: 160-167.

23. FAO/WHO: Health and nutritional properties of probiotics in food including powder milk with live lactic acid bacteria. WHO 2001.

24. Hill C, Guarner F, Reid G, Gibson GR, Merenstein DJ, Pot B, et al: Expert consensus document. The International Scientific Association for Probiotics and Prebiotics consensus statement on the 
scope and appropriate use of the term probiotic. Nat Rev Gastroenterol Hepatol 2014; 11: 506-514.

25. FAO: FAO Technical Meeting on prebiotics. Food Quality and Standards Service (AGNS). September 15-16, 2007.

26. ISAPP: 6th Meeting of the International Scientific Association of Probiotics and Prebiotics. London, Ontario, 2008.

27. FAO/WHO: Joint FAO/WHO working group report on drafting guidelines for the evaluation of probiotics in food. FAO, 2002.

28. Guarner F, Requena T, Marcos A: Consensus statements from the Workshop "Probiotics and Health: Scientific evidence". Nutr Hosp 2010; 25: 700-704.

29. Rodríguez JM: Probióticos: del laboratorio al consumidor. Nutr Hosp 2015; 31(S1): 33-47.

30. Álvarez-Calatayud G, Marcos A, Margolles A, Editors: Probióticos, probióticos y salud: Evidencia científica. Ergón, Madrid, 2016.

31. EFSA Panel on Dietetic Products, Nutrition and Allergies: Scientific Opinion on the substantiation of health claims related to live yoghurt cultures and improved lactose digestion (ID 1143, 2976) pursuant to Article 13(1) of Regulation (EC) No 1924/2006. EFSA J; 2010; 8: 1763

32. NASPGHAN Nutrition Report Committee, Michail S, Sylvester $F$, Fuchs $G$, Issenman $R$. Clinical efficacy of probiotics: review of the evidence with focus on children. J Pediatr Gastroenterol Nutr 2006; 43: 550-557.

33. Szajewska H, Guarino A, Hojsak I, Indrio F, Kolacek S, Shamir $R$, et al: European Society for Pediatric Gastroenterology, Hepatology, and Nutrition. Use of probiotics for management of acute gastroenteritis: a position paper by the ESPGHAN Working Group for Probiotics and Prebiotics. J Pediatr Gastroenterol Nutr 2014; 58: 531-539.

34. Guarino A, Guandalini S, Lo Vecchio A: Probiotics for prevention and treatment of diarrhea. J Clin Gastroenterol 2015; 49 Suppl 1: S37-45.

35. Goldenberg JZ, Lytvyn L, Steurich J, Parkin P, Mahant S, Johnston BC: Probiotics for the prevention of pediatric antibiotic-associated diarrhea. Cochrane Database Syst Rev. 2015; CD004827.

36. Szajewska H, Canani RB, Guarino A, Hojsak I, Indrio F, Kolacek $S$, et al: Probiotics for the prevention of antibiotic-associated diarrhea in children. J Pediatr Gastroenterol Nutr 2016; 62: 495-506.

37. Gionchetti P, Calabrese C, Lauri A, Rizzello F: The therapeutic potential of antibiotics and probiotics in the treatment of pouchitis. Expert Rev Gastroenterol Hepatol. 2015; 9: 1175-1181.

38. Lichtenstein L, Avni-Biron I, Ben-Bassat O; The current place of probiotics and prebiotics in the treatment of pouchitis. Best Pract Res Clin Gastroenterol. 2016; 30: 73-80.

39. Reid G: Probiotic and prebiotic applications for vaginal health. J AOAC Int 2012; 95: 31- 34.

40. Hanson L, VandeVusse L, Jermé M, Abad CL, Safdar N: Probiotics for treatment and prevention of urogenital infections in women: a systematic review. J Midwifery Womens Health 2016; 61:339-355.

41. Stapleton AE, Au-Yeung M, Hooton TM, Fredricks DN, Roberts PL, Czaja CA, et al: Randomized, placebo-controlled phase 2 trial of a Lactobacillus crispatus probiotic given intravaginally for prevention of recurrent urinary tract infection. Clin Infect Dis 2011; 52: 1212-1217.

42. Beltrán D, Guerra J: Consenso en probióticos vaginales. EDIMSA, Madrid, 2012.
43. Fernández L, Langa S, Martín V, Maldonado A, Jiménez E, Martín R, et al: The human milk microbiota: Origin and potential roles in health and disease. Pharmacol Res. 2013; 69: 1-10.

44. Soto A, Martin V, Jimenez E, Mader I, Rodriguez JM, Fernandez L: Lactobacilli and bifidobacteria in human breast milk: Influence of antibiotherapy and other host and clinical factors. J Pediatr Gastroenterol Nutr 2014; 59: 78-88.

45. Arroyo R, Martín V, Maldonado A, Jiménez E, Fernández L, Rodríguez JM: Treatment of infectious mastitis during lactation: antibiotics versus oral administration of lactobacilli isolated from breast milk. Clin Infect Dis 2010; 50: 1551-1558.

46. Fernández L, Arroyo R, Espinosa I, Marín M, Jiménez E, Rodríguez JM: Probiotics for human lactational mastitis. Benef Microbes 2014; 5: 169-183.

47. Fernández L, Cárdenas N, Arroyo R, Manzano S, Jiménez E, Martín V, et al: Prevention of infectious mastitis by oral administration of Lactobacillus salivarius PS2 during late pregnancy. Clin Infect Dis 2016; 62: 568-573.

48. Ubeda C, Bucci V, Caballero S, Djukovic A, Toussaint NC, Equinda $M$, et al: Intestinal microbiota containing Barnesiella species cures vancomycin-resistant Enterococcus faecium colonization. Infect Immun. 2013; 81: 965-973.

49. Buffie CG, Bucci V, Stein RR, T, Ling L, Gobourne A, No D, et al: Precision microbiome reconstitution restores bile acid mediated resistance to Clostridium difficile. Nature 2015; 517: 205-208.

50. Martín R, Miquel S, Benevides L, Bridonneau C, Robert V, Hudault $S$, et al: Functional characterization of novel Faecalibacterium prausnitzii strains isolated from healthy volunteers: a step forward in the use of F. prausnitzii as a next-generation probiotic. Front Microbiol 2017; 8: 1226.

51. Bakken JS, Borody T, Brandt LJ, Brill JV, Demarco DC, Franzos MA, et al: Treating Clostridium difficile infection with fecal microbiota transplantation. Clin Gastroenterol Hepatol 2011; 9: 10441049.

52. Gough E, Shaikh H, Manges AR: Systematic review of intestinal microbiota transplantation (fecal bacteriotherapy) for recurrent Clostridium difficile infection. Clin Infect Dis 2011; 53: 994-1002.

53. van Nood, E., Vrieze, A., Nieuwdorp, M., Fuentes, S., Zoetendal, E. G., de Vos, W. M., et al: Duodenal infusion of donor feces for recurrent Clostridium difficile. New Engl J Med 2013; 368: 407-415.

54. Dominguez-Bello MG, De Jesus-Laboy KM, Shen N, Cox LM, Amir A, Gonzalez A, et al: Partial restoration of the microbiota of cesarean-born infants via vaginal microbial transfer. Nat Med 2016; 22: 250-253.

55. Petrof EO, Gloor GB, Vanner SJ, Weese SJ, Carter D, Daigneault MC, et al: Stool substitute transplant therapy for the eradication of Clostridium difficile infection: 'RePOOPulating' the gut. Microbiome 2013;1: 3. 


\section{MICROBIOTA, PROBIOTICS AND THE FIGHT AGAINST ANTIBIOTIC-RESISTANT MICROOR- GANISMS: THE VETERINARY SIDE}

\author{
${ }^{(1)}$ G. Pérez Martínez, ${ }^{(2)}$ T. Requena, ${ }^{(3)}$ O.J. Sobrino, ${ }^{(4)}$ J.M. Rodríguez. \\ (1)Departament of Biotechnology, Agrochemistry and Food \\ Technology Institute (IATA-CSIC). Spanish National Research \\ Council (CSIC). Valencia. Spain; ${ }^{(2)}$ Institute of Food Science \\ Research (CIAL-CSIC). Madrid. Spain. CIAL (CSIC-UAM), Madrid. \\ Spain; ${ }^{(3)}$ The Ministry of Agriculture and Fisheries, Food and \\ Environment. Madrid. Spain; ${ }^{(4)}$ Department of Nutrition and \\ Food Science. Complutense University of Madrid. Spain.
}

\section{Corresponding:}

\section{Juan M. Rodríguez, PhD}

Professor

Department of Nutrition and Food Science, Complutense University of Madrid, Avda. Puerta de Hierro s/n., 28040 Madrid, Spain

E-Mail jmrodrig@ucm.es

\section{ABSTRACT}

Antibiotics have contributed significantly to the improvement of animal health and to the availability of animal-derived foodstuffs. However, the routine use of antimicrobials in animal production has raised concerns not only about the potential presence of residues in foods but, particularly, about the development and spread of antibiotic-resistant bacteria that may compromise the treatment of animal and human infectious diseases. In this context, strategies to reduce antibiotic use and/or mitigate their collateral damage, especially the development of resistance and adverse effects on the host microbiota, must be developed. In this article, different strategies and lines of action or research are proposed to be applied within the framework of the Spanish National Plan for Antibiotic Resistance.

Key Words: microbiota / animal nutrition/ probiotics / infections /antibiotics/ antibiotic resistance.

\section{INTRODUCTION}

Expansion of antibiotic resistances has generated an unprecedented public health problem that is responsible for a rapidly increasing number of deaths due to bacterial infections that had been controlled in the past [1], a progression that may lead to situations where a simple cold or a superficial wound could lead to life-threatening infections.

Spreading of antibiotic resistances has been favored by different factors. First, by an extensive prescription of broad-spectrum antibiotics, possibly related to the current unavailability or low use of fast methods to determine the most appropriate antibiotic in a case-bycase basis. Self-medication and global high mobility of goods and people have favoured expansion of resistances, and also the widespread use of antibiotics in niches with high bacterial density and easy transmission between individuals, such as hospitals, where the use of antibiotics is often fully justified, and intensive farms.

\section{ANTIBIOTICS IN ANIMAL FARMS: FROM HIGHER PRODUCTION TO PUBLIC HEALTH CONCERN}

Antibiotics have not only saved millions of human lives but, also, their use has significantly contributed to the improvement of animal health. The use of antibiotics in livestock has resulted in healthier animals and the production of large quantities of nutritious, high-quality, and low-cost food for human consumption. Despite these benefits, the authorities responsible for public health and food safety have repeatedly expressed great concern about the routine use of antimicrobials in livestock farms and, more specifically, about the development of resistances, which may compromise the future treatment of human and animal diseases that require antibiotic intervention. In this regard, it should be remembered that the molecules of many of the antibiotics used in both human and animal health are very similar and that, in some cases, the same antibiotics have been used in both "worlds".

Shortly after the antibiotics began to be used to treat human and animal infections, it was discovered that the administration of low (subtherapeutic) doses of antibiotics to cattle promoted their growth and that the effect was greater more lasting when the exposure was earlier in life [2]. Since then, the use of antibiotics has been generalized in intensive farms, either for prophylactic or metaphylactic use, or as growth promoters frequently exceeding advisable limits and confronting national and international recommendations. Intensive production systems create the ideal conditions for bacteria to acquire and fix the genes that confer antibiotic resistance. The demonstration of the transfer of a plasmid that confers multi-resistance to antibiotics (ampicillin, amoxicillin-clavulanic acid, cephalosporins, chloramphenicol, spectinomycin and sulfisoxazole) from a strain of E. coli O168: $\mathrm{H} 7$ to other strains of E. coli and Salmonella serovar Newport in the digestive tract of farm turkeys [3] is an illustrative example. Such genes may be subsequently transmitted to human pathogens or to the human intestinal microbiota. The direction of transmission is an important issue to be investigated since resistances are transferred to humans from food, but also farm animals and animal-derived foods (e.g., meat during slaughtering and subsequent manipulations) can get resistant bacteria from humans. A recent study found that all strains of Enterococcus faecium isolated from chicken meat in a North American state carried multi-resistance to antibiotics and all of them were resistant to quinupristin/dalfopristin, a product routinely used among the hospitals of the same state to treat infections caused by vancomycin-resistant strains [4]. In another study in which the presence of Staphylococcus aureus in chicken samples was analysed, it was observed that the two isolates resistant to methicillin were of human origin [5].

\section{THE “ONE WORLD, ONE HEALTH” APPROACH}

The "One World, One Health" approach, defined as "the collaborative effort of multiple disciplines - working locally, nationally and globally - to achieve optimal health for people, animals and our environment" [6], recognizes that the health of people is connected to the health of animals (especially that of food-producing animals and pets) and to a healthy environment. It is difficult to imagine a problem that exemplifies the principles of this approach better than resistance to antimicrobials [7]. With a high consumption of antimicro-

9th Workshop SEPyP 
bials in animal production, it seems likely that agricultural use has an important contribution to antimicrobial resistance spreading [8-10].

Many human and animal infections have an endogenous origin and are caused by microorganisms that are part of the individual's own microbiota and that, under certain circumstances, act as opportunistic pathogens and can lead to disease. In this sense, the possible transmission of antibiotic-resistance genes between the normal microbiota of animals and that of humans is an eminently unknown issue that deserves to be investigated [7] (Fig. 1). A disturbing example is the recent appearance of an E. coli strain carrying a colistin-resistance gene in humans and pigs in China [11]. Colistin is a last resort antibiotic in human medicine but its use is widespread in animal (mainly porcine) production. In this particular case, it involves a plasmid-mediated resistance that has rapidly spread in Europe, including Spain, and North America [12-15].

The amount of antibiotics used in global livestock production is estimated to be higher than 63,000 tons per year $[16,17]$, suggesting that the consumption of antibiotics in the agricultural sector notably exceeds direct human consumption. The last report of the European Medicines Agency [18] on sales of antibiotics for use in the veterinary field shows that Spain is at the head of the 29 European countries that provided data $(419 \mathrm{mg} / \mathrm{kg}$ of meat produced, when the European average is $121 \mathrm{mg} / \mathrm{kg}$ ), so, overall, our country is in a particularly worrying situation, with the aggravating circumstance that, as stated above, one of the most employed ones (colistin) is a last resort antibiotic in human medicine.

Despite all the above, some professionals in the animal production sector firmly believe that the advantages of using antibiotics far outweigh the disadvantages. In this sense, they believe that the issue of the prohibition or not of the use of antimicrobials in slaughter animals is complex and controversial, and that the debate should include the question of what would happen if the extensive use of antibiotics was banned for use in food-producing animals [19]. The implications of the response include aspects such as human health, welfare, and animal health, as well as the effects on the quantity, quality, and cost of food. An exhaustive review was aimed to find significant evidences of the link between antibiotic use in animals and the arousal of antibiotic resistances in humans [20]. Author reported that out of 280 peer-reviewed scientific publications dealing with the transmission of antibiotic resistances from farms to humans, a high proportion of publications did not take a clear stance or yielded not applicable studies suggesting that further investigation is needed; however, 114 (41\%) publications stated that there is a significant relationship between antibiotic use in agriculture and emergence of antibiotic resistances in humans, while only 15 (5\%) demonstrated the opposite (Fig. 2). Interestingly, the vast majority of articles published from universities reported in the direction of a positive influence of the use of antibiotics on the rise of human resistances. In any case, it seems clear that, at least in intensive systems, the use of antibiotics is disproportionately high and that this routine practice can cause serious damage to public health, so its use should be monitored and alternative strategies promoted.

The third pillar of Health, environmental health (including that of crops where antimicrobials are also used in plant protection [21]), is the least known in the process of evolution, persistence and transmission of the resistance genes. Environmental bacteria, which are quantitatively the most frequent organisms, serve

9th Workshop SEPyP as sources of antimicrobial resistance genes that can be acquired, over time, by human and animal pathogens. This natural phenomenon is exacerbated by the influx into the environment of the genes existing in human and livestock waste and effluents [22], and by the large amounts of antibiotic residues that are incorporated into the environment from the pharmaceutical industries [23], intensive livestock farms [24] and hospitals [25] (Fig. 1).

The Council of the European Union, the European Parliament, the European Commission and its Agencies (EMA, ECDC, HMA, EFSA) have identified the need to establish a common European strategy to assess and address the problem of the development of resistance to antibiotics. This has been shown in various official documents, such as the conclusions of the Council of the European Union of May 29, 2012 on the impact of antibiotic resistance, in which Member States were urged to develop and implement National level strategies or action plans to contain the development of antibiotic resistance, manifesting the need for a joint human and veterinary perspective if these strategies are to be truly effective. The multidisciplinary nature of the members of the SEPyP (Sociedad Española de Probióticos y Prebióticos) places this society as an ideal frame to make proposals that globally encompass these both areas of Health (human, animal).

\section{ANTIBIOTICS IN THE SWINE, POULTRY AND RABBIT PRODUCTION}

Intensive porcine sector is one of the main consumers of antibiotics, among which the use of $\beta$-lactamics (amoxicillin) and polymyxins (colistin) is particularly high. Antibiotic consumption is especially relevant during the stages of weaning and transition [26], so that a decrease in the use of antimicrobials in these stages will significantly reduce their overall consumption. In the European Union (EU), piglets' weaning takes place between 2128 days postpartum, just at the moment when the digestive system is in the process of development and the animal's immune system is still very immature. In addition, during the weaning period there are abrupt and stressful changes in feeding and handling (separation of the mother, regrouping, transfers). All this leads to a profound alteration of the digestive microbiota, which is associated to the destruction of intestinal villi, a decrease in feed intake, a decrease in weight gain and the proliferation of certain pathogens that, ultimately, cause diarrhoea and respiratory processes. The use of antimicrobials in starter feeds is intended to prevent the development of these pathogens, a practice that causes a selection of the resistant clones and the establishment of a vicious circle (antibiotics-selection of resistant bacteria) in the exploitation. In fact, the increasing appearance of resistance to antimicrobials may be the cause of the syndrome known as the new neonatal porcine diarrhoea in piglets [27].

As noted above, the situation is similar in other species that are exploited in intensive regimes, including poultry farms [28], another sector very relevant to the Spanish economy. As for other species, such as rabbits, recent data suggest that epizootic enteropathy, a devastating intestinal disease that causes between 30 and $95 \%$ of mortality in the post-weaning period and whose aetiology is still unknown, could be related precisely with the anomalies in the intestinal populations generated by the continuous administration of antibiotics [29]. 


\section{ANTIBIOTICS FOR THE PREVENTION AND TREATMENT OF MASTITIS IN CATTLE}

Special mention deserves the empirical use of antibiotics for the treatment of mastitis in ruminants, mainly in cattle. In general, antibiotics are intramammary applied for the treatment of mild and moderate cases, also resorting to the parenteral route in the most severe cases. An increase in the incidence of the disease in a herd usually results in an increased use of antimicrobials, which in turn increases the amount of antibiotic residues in the milk and favours the acquisition of antimicrobial resistance. In addition, antibiotics are often routinely administered to whole herds to prevent mastitis during the dry period [30]. In field conditions, the use of certain antimicrobials for the treatment and control of mastitis, such as penicillin, pirlimycin or florfenicol, is positively associated with antimicrobial resistance among the pathogens that cause bovine mastitis [31]. Despite this, some researchers believe that the human clinical consequences derived from antimicrobial resistance among pathogens that are prevalent in dairy cattle and dairy environments are small [19]. Perhaps this opinion is due, to a large extent, to the scarcity of studies on the subject since there are several potential routes for the transmission of such microorganisms to humans (transfer to skin and mucous membranes of farmers and veterinarians, consumption of milk and dairy products not subjected to heat treatment or re-contaminated, consumption of meat and meat products, dissemination from dairy farms effluents, etc.).

Consequently, it is a sector in which the availability of alternatives to antibiotics would be particularly appealing [32]. These include the use of intramammary probiotics, an application that seems effective [33-36] but faces a decisive legal barrier since, at least within the EU, all intramammary products must be sterile (just the opposite of what would involve the administration of a live probiotic microorganisms). On the other hand, it is essential to encourage the prudent use of antibiotics in milk production systems and restrict their use to situations in which they are strictly necessary. In such instances, identification of the pathogen(s) that cause(s) the infection and the determination of their antibiotic susceptibility (to evaluate the most appropriate antibiotic in each case) would be highly recommended.

\section{ANTIBIOTICS IN AQUACULTURE}

In 2013, fish and shellfish production through aquaculture practices was higher, for the first time, than that obtained through fishing. Therefore, aquaculture is not a mere complement to fishing but probably represents the animal farming sector with the greatest future projection. It has in its favour that $70 \%$ of the surface of Earth is water, it does not require the consumption of fresh water, and aquatic animals are more efficient food-weight converters and their reproduction rates are several orders of magnitude higher than these of terrestrial vertebrates. Spain is the EU Member State with the largest volume of production in aquaculture, although when considering its economic value it falls to fifth place [37]. This loss of positions is due to the fact that mussels, which are the main Spanish aquaculture species, have a lower unit value than other species, especially fish. In any case, it is a strategic sector for Spain, a country that has a varied availability of water resources on which it is possible to carry out aquaculture, both in marine and continental areas [37].
Bacterial infections are a frequent cause of morbidity and mortality in intensive aquaculture, with concomitant decreases in production [38]. The control of infections through vaccination (little developed in this sector) and the improvement of hygienic conditions constitute the best long-term approach; however, the incorporation of antimicrobials into feed (medicated feed) is the current primary tool for the prevention and treatment of bacterial infections [39-41]. Logically, antibiotics and their metabolites present in non-ingested food, along with those eliminated in the urine and faeces of the animals, end up in the surrounding environment [42]. The passage of antimicrobials to the environment can further contribute to the selection of resistant bacteria and to increase the horizontal transfer of resistance genes.

There is evidence of, at least, some potential effects of the excessive use of antimicrobials in aquaculture [42, 43]. In a study that evaluated the impact of the use of tetracycline in Norwegian aquaculture, it was observed that the bacteria existing in the sediment of the aquaculture farms were $100 \%$ resistant to that antibiotic immediately after its application, and the resistance rate fluctuated between $10 \%$ and 50\% during the following 18 months [44]. In comparison, the rate of resistance to tetracycline was less than $1 \%$ among the bacteria in the sediment of a place where such activity was not practiced [44]. In a Danish study on the use of antimicrobials in freshwater rainbow trout holdings, high rates of resistance to tetracycline, sulfonamides/trimethoprim, oxolinic acid, amoxicillin and florfenicol were found in bacteria isolated from water, sediments and fish [45]. More recently, significant increases in the number of bacteria resistant to oxytetracycline, oxolinic acid and florfenicol have been found in the sediments of marine aquaculture farms compared to those of areas where aquaculture is not practiced [46]. In addition, it has been observed that the dispersion of the large quantities of antimicrobials used in Chilean salmon aquaculture has created a selective pressure even in areas of the marine environment far from the initial site where these agents were used [47].

\section{ANTIBIOTICS IN PETS AND RECREATIONAL ANIMALS}

Until approximately a decade ago, the risks of the use of antimicrobials in the treatment of infections in companion animals (dogs, cats) or recreational animals (horses) were assumed to be low for the human species. However, publications that have appeared since then indicate that this topic is much more relevant than it was supposed to be and deserves more attention from epidemiological and public health services [48]. Isolation of antibiotic-multiresistant Acinetobacter baumannii, S. aureus, Staphylococcus intermedius, E. coli or Salmonella enterica strains (including methicillin-resistant $S$. aureus) as agents of nosocomial infections in dogs hospitalized in intensive care units seems to be increasing [49]. The authors suggested that this outcome could reflect the abundant use of broad-spectrum antibiotics in such units. The transmission of such organisms occurs between pets, owners and veterinary personnel, and all of them can act as reservoirs, in a process called "community transmission". In 2003, Manian [50] described a case of recurrent infection by a methicillin-resistant $S$. aureus strain in a human patient with diabetes and in his wife, which could only be resolved when it was discovered that their dog shared the same strain and was consequently treated. The role

9th Workshop SEPyP 
of dogs, cats and horses as reservoirs of this and other types of bacterial species that are resistant to antibiotics and relevant to human health seems increasingly relevant [51].

It should be remembered that, in general, the drugs used in the treatment of sick pet animals are practically the same as those employed in human medicine, with an increasing use of aminopenicillins, cephalosporins and fluoroquinolones; however, this trend is subject to recent review under the categorization of antibiotics for veterinary use (see below).

\section{CAN THE USE OR THE IMPACT OF ANTIBIOTICS BE REDUCED OR MITIGATED?}

There is no doubt that antibiotics have revolutionized medical care and are still essential for our society. There will always be cases in which children, adults or animals should be treated with an antibiotic but strategies to reduce their use and/or mitigate their collateral damage, especially the development of resistance and adverse effects on the individual's microbiota, must be developed. These include the use of our growing knowledge on microbial genomes and human and animal metagenomes for the development of specific drugs against specific pathogens, in contrast to the indiscriminate "bombardment" associated with current broad-spectrum antibiotics. Different supranational (e.g., $\mathrm{WHO}$ ) and national organizations worldwide have pronounced on this [52]. From the list of critically important, very important and important antibiotics for human health prepared by the WHO [53], different measures are currently being implemented in some regions. For example, the European Medicines Agency (EMA) by request of the European Commission, proposed that antibiotics in the list of critically important antibiotics currently licensed for veterinary medicine should be divided in two categories: Category 1 (narrow spectrum penicillins, macrolides, tetracyclines) classified as low/limited risk to public health for which general principles of responsible use should be applied; and Category 2 (aminoglycosides, 3rd and 4thgeneration cephalosporins, fluoroquinolones, glycopeptides and colistin) for which a high risk for public health is assumed and must have a restricted use where there are no alternatives. This was included in the 2016-2020 global strategy of the EMA [54] and communicated to all the different EU member states. Several other countries and professional organizations have prepared measures or warning documents proposing similar lines of action $[21,55,56]$.

These formal recommendations need the involvement of the veterinaries and the production sectors. Contrary to prevalent concepts, possibly the extensive antibiotic use, in addition to the public health issue, may be perpetuating some inefficient farming practices. In fact, recent studies have provided evidences to clearly demonstrate that reduction of antimicrobial administration can also be beneficial for the farmer. In Ireland and The Netherlands, farmers, veterinaries, nutritionists and external advisors have developed measures that involve changes in nutrition (lower protein), management systems-like "all in, all out", vaccination programmes, feed supplements like probiotics and prebiotics and others that assure a high quality production and rational and profitable management of the herds $[57,58]$. Essentially, seeking animals' well-being and health reduces antibiotic need and increases profits.

9th Workshop SEPyP
In addition, by reducing antibiotic intake the damage to the symbiotic microbial species will be minimized and the structure and function of the microbial community of the host will be preserved. In the future, interindividual variability within the same species may require more host-personalized approaches for antibacterial therapies (choice of product, route of administration, posology, treatment time, etc.).

These strategies also require a better knowledge of the pathogenesis of most infections and the availability of better diagnostic tests that allow, in a quick, accurate and economically feasible manner, to (a) differentiate bacterial infections from fungal and viral infections; (b) identify the bacteria that are responsible for infections and know properties relevant to the treatment, such as their antibiotic sensitivity or biofilm-forming ability; (c) distinguish between colonization and infection; and (d) identify early markers of infection, which make it possible to avoid or reduce the use of prophylactic antibiotherapy.

A greater knowledge of the composition, functions and alterations of the human and animal microbiota, microbiome and metagenome will also be fundamental. Some authors have urged more robust clinical trials to assess the short and long-term impact of antibiotic exposures. In this way it would be possible to identify which are, globally, the key microorganisms in a healthy microbiome and lay the foundations to be able to rebuild the affected ecosystem through changes in diet, the use of prebiotics and/or the prescription of well characterized probiotic strains in pharmaceutical quality preparations [59-61] (see previous article in this issue). Obviously, it is a difficult task that requires a great research and clinical effort because, as discussed previously, the human and animal microbiota is extremely variable between individuals and over time, especially in the first years of life.

In addition, it must be considered that future probiotics may include some of the strict anaerobic bacteria (Faecalibacterium spp., Roseburia spp., Ruminococcus spp., Eubacterium spp., Bacteroides spp., among others) that seem to characterize the microbiota of healthy hosts. The difficulty in the growth and stability of this kind of bacteria at an industrial scale makes their application not feasible from a technological and economic point of view at present. However, culturomic advances can make the situation change in a not too distant future.

Faecal transfer (also called faecal transplant or faecal bacteriotherapy) (see previous article in this issue) is another of the current lines of work and has aroused great interest in human medicine as a method to modify complex microbial ecosystems in pathological situations. The main reason for the popularity of faecal transplants is the high efficiency that has been shown in the treatment of certain diseases that are very difficult to control and, especially, in the recurrent infection by Clostridium difficile $[62,63]$. However, this concept has been empirically applied in animal production for many centuries. In 1973, Finnish researchers Nurmi and Rantala [64] demonstrated that the transfer of faecal material from adult chickens to newly hatched chickens conferred an almost instantaneous protection against Salmonella colonization and infection (the so-called "Nurmi effect"). Since then, several well-designed and controlled studies have shown a similar protective effect against infections by E. coli, Campylobacter jejuni or Clostridium perfringens and products containing freeze-dried preparations of faecal microbiota from pathogen-free chicken have been stan- 
dardized, patented and commercialized [65-67]. This approach is similar to the concept of minimal or synthetic microbiotas recently proposed in relation to the restoration of the human microbiota $[68,69]$, and may be applied to other farm species.

In fact, similar strategies have been proposed or empirically used, in the porcine sector including: (a) the use of defined mixtures of microorganisms for early administration to piglets [70]; (b) the transfer of intestinal material from piglets with diarrhoea to pregnant sows with the objective of actively stimulating their immune system against E. coli and other bacterial agents that may cause diarrhoea at weaning; subsequently, maternal immunity would be passively transferred to foetuses or piglets during late gestation or lactation, respectively ("feed back" technique); and (c) the selection of pathogen-free donor pigs for the transfer of their faecal microbiota to weaned piglets during the transition period ("feed forward" technique).

The concept of transferring biological samples in veterinary practice dates from, at least, the 17th century, in relation to the transfer of ruminal material ("rumen transfaunation"), a common practice to treat certain digestive diseases of cattle [71]. Transfaunation refers to the transfer of a broad spectrum of microorganisms, including bacteria, protozoa, fungi and archaea, from the rumen of a healthy donor animal to the rumen of a diseased recipient animal. This procedure is experiencing a new boom because of new scientific reports showing therapeutic efficacy in several ruminal and gastrointestinal disorders, including acidosis of cows fed high grain levels [72]. In addition, it allows reducing or avoiding the use of several drugs, including antibiotics [71].

Faecal transplantation faces important practical problems in human clinical practice, derived from the extraordinary microbiological, immunological and biochemical complexity of faeces, whose composition can vary even within the same person, depending on numerous factors. For example, faeces could become a source of harmful substances or microorganisms that may represent a health problem in the medium and long term. This problem seems not very relevant in food animals under intensive production systems since (except for dairy cattle and selected animals destined to reproduction) they are usually sacrificed at very early ages.

On the other hand, it is a biological sample that is impossible to standardize, which limits its application on a large scale. In this regard, it has been suggested that biobanks should preserve faecal samples from healthy children in order to transfer their own microbiota in the future, after treatment with antibiotics or in certain pathological situations. Biobanks including faeces from healthy food animal and pets could be also a future possibility, which may be feasible even at an in-farm level.

The implementation of the microbial communities in next-generation therapies would be of great benefit to patients and, in addition, would advance our understanding of the animal intestinal microbiome. Many studies will be necessary to define the functions that the components of a microbiome should play for the prevention or treatment of infections and other conditions in which microbiome is affected. In any case, the development of defined microbial communities, representative of those that exist in a determined niche under physiological conditions, supposes an attractive approach - not exempt of a great technical difficulty - for the prevention and treatment of infections in the context of the increasing appearance of multiresistant pathogenic bacteria.

\section{CONCLUSIONS AND RESEARCH PROPOSALS}

The globalization of the agri-food system, with an unprecedented increase in the movement of animals and food, combined with the spectacular increase in human travel, facilitates the rapid spread of emerging resistance genes. No country is protected against the entry of high risk antibiotic-resistant clones. Antimicrobial resistance is a global problem that requires global solutions, but the solutions will not be the same in all countries, nor among the different socioeconomic groups. Some use them excessively, others do not have access to them and many use them recklessly; the understanding of the patterns of use, the incentives to change them and the exploration of alternative options should sustain any reduction effort.

Within the framework of the Spanish National Plan for Antibiotic Resistance, the Spanish Society for Probiotics and Prebiotics (SEPyP) proposes the following lines of action/research:

(1) To develop efficient molecular methods for the determination of antibiotic resistance genes in farms for an efficient antibiotic use and to increase our knowledge on the molecular epidemiology of infections caused by antibiotic-resistant bacteria and on the routes and mechanisms for potential animal-food-human transmission

(2) To study the microbiota, microbiome and metagenome of food animal species (and derived foods) and pets in health and disease to determine biochemical, immunological and microbiological biomarkers associated with risk of disease, and to know the prevalence of antibiotic-resistance bacteria/genes and their potential transfer to the human species.

(3) To isolate, purify, identify, select, characterize and/or produce prebiotics, probiotics and synbiotics able to restrain opportunistic pathogens and compete with invasive pathogens, to substitute or complement antibiotics in the treatment and/or prevention of infections or other microbiome-involved diseases. To define the criteria and procedures for evaluating their effectiveness and safety (including the safety and sensorial properties of foods obtained from treated animals).

(4) To define criteria for the selection of donors and recipients for faecal/ruminal (or another type of biological material) transfer with the objective of modulating the microbiota and replacing or complementing antibiotics in the treatment and/or prevention of infections or other microbiome-involved diseases. To define the criteria and procedures for evaluating their effectiveness and safety (including the safety and sensorial properties of foods obtained from treated animals).

(5) To define criteria for the selection, transfer and production of minimal or synthetic microbiota with the aim of modulating the microbiota and replacing or complementing antibiotics in the treatment and/or prevention of infections or other microbiome-involved diseases. To define the criteria and procedures for evaluating their effectiveness and safety (including the safety and sensorial properties of foods obtained from treated animals).

(6) To identify dietary factors and farming practices capable of modulating the structure of the microbiota during any stage of life in order to replace or complement the antibiotics in the treatment and/ or prevention of infections or other microbiome-involved diseases.

(7) To promote normative changes that may allow or facilitate the use as probiotics of microorganisms that seem to be particular- 
ly beneficial but that are not currently in the list of QPS or GRAS microorganisms.

(8) To promote regulatory changes that may allow or facilitate the use of probiotics in areas where they cannot be applied with current legislation, such as intramammary administration for the prevention or treatment of mastitis.

\section{REFERENCES:}

1. Ferri M, Ranucci E, Romagnoli P, Giaccone V: Antimicrobial resistance: A global emerging threat to public health systems. Crit Rev Food Sci Nutr 2017; 57: 2857-2876.

2. Zimmerman DR: Role of subtherapeutic levels of antimicrobials in pig production. J Anim Sci 1986; 62 (Suppl 3): 6-16.

3. Poppe C, Martin LC, Gyles CL, Reid-Smith R, Boerlin P, McEwen $S A$, et al: Acquisition of resistance to extended-spectrum cephalosporins by Salmonella enterica subsp. enterica serovar newport and Escherichia coli in the turkey intestinal tract. Appl Environ Microbiol 2005; 71: 1184-1192.

4. Limayem A, Donofrio RS, Zhang C, Haller E, Johnson MG: Studies on the drug resistance profile of Enterococcus faecium distributed from poultry retailers to hospitals. J Environ Sci Health B 2015; 50: 827-832.

5. Abdalrahman LS, Stanley A, Wells H, Fakhr MK: Isolation, virulence, and antimicrobial resistance of methicillin-resistant Staphylococcus aureus (MRSA) and methicillin sensitive Staphylococcus aureus (MSSA) strains from Oklahoma retail poultry meats. Int J Environ Res Public Health 2015; 12: 6148-6161.

6. AVMA: One health: A new professional imperative. One Health Initiative Task Force Final Report, American Veterinary Medical Association, Schaumburg, 2008.

7. Robinson TP, Wertheim HFL, Kakkar M, Kariuki S, Bu D, Price LB: Animal production and antimicrobial resistance in the clinic. Lancet 2016; 387:e1-e3.

8. Hu Y, Yang X, Lu N, Zhu B: The abundance of antibiotic resistance genes in human guts has correlation to the consumption of antibiotics in animal. Gut Microbes 2014;5:245-249.

9. Holmes AH, Moore LSP, Sundsfjord A, Steinbakk M, Regmi $S$, Karkey A, et al: Understanding the mechanisms and drivers of antimicrobial resistance. Lancet 2016; 387: 176-187.

10. Robinson TP, Bub DP, Carrique-Masc J, Fèvred EM, Gilberte $M$, Gracea D, et al: Antibiotic resistance is the quintessential One Health issue. Trans R Soc Trop Med Hyg 2016; 00: 1-4.

11. Liu Y-Y, Wang T, Walsh TR, Yi L-X, Zhang R, Spencer J, et al: Emergence of plasmid-mediated colistin resistance mechanism MCR-1 in animals and human beings in China: a microbiological and molecular biological study. Lancet Infect Dis 2016; 16: 161-168.

12. Hasman H, Hammerum A, Hansen F, Hendriksen R, Olesen $B$, Agers $\varnothing Y$, et al: Detection of mcr-1 encoding plasmid-mediated colistin-resistant Escherichia coli isolates from human bloodstream infection and imported chicken meat, Denmark 2015. Euro Surveill. 20(49):pii=30085.

13. McGann P, Snesrud E, Maybank R, Corey B, Ong AC, Clifford $R$, et al: Escherichia coli harboring $m c r-1$ and blaCTX-M on a novel IncF plasmid: First report of mcr-1 in the USA. Antimicrob Agents Chemother 2016;60:4420-4421.

9th Workshop SEPyP
14. Haenni M, Poirel L, Kieffer N, Châtre P, Saras E, Métayer V, et al: Co-occurrence of extended spectrum $\beta$ lactamase and MCR-1 encoding genes on plasmids. Lancet Infect Dis 2016; 6: 281-282.

15. Quesada A, Ugarte-Ruiz M, Iglesias $M R$, Porrero $M C$, Martínez R, Florez-Cuadrado D, et al: Detection of plasmid mediated colistin resistance (MCR-1) in Escherichia coli and Salmonella enterica isolated from poultry and swine in Spain. Res Vet Sci 2016; 105: 134-135.

16. Van Boeckel TP, Brower C, Gilbert M, Grenfell BT, Levin SA, Robinson TP, et al: Global trends in antimicrobial use in food animals. Proc Natl Acad Sci 2015; 112: 5649-5654.

17. Henriksson PLK, Troell M, Rico A: Antimicrobial use in aquaculture: Some complementing facts. Proc Natl Acad Sci 2015; 112: E3317.

18. EMA: Sales of veterinary antimicrobial agents in 29 European countries in 2014 Sixth ESVAC report EMA/61769/2016. 2016.

19. Oliver SP, Murinda SE, Jayarao BM: Impact of antibiotic use in adult dairy cows on antimicrobial resistance of veterinary and human pathogens: a comprehensive review. Foodborne Pathog Dis 2011; 8: 337-355.

20. Stockwell VO, Duffy B: Use of antibiotics in plant agriculture. Rev Sci Tech Off Int Epiz 2012; 31: 199-210.

21. O'Neill, J (chair): Antimicrobials in agriculture and the environment: Reducing unnecessary use and waste: The review on antimicrobial resistance. Wellcome Trust, 2015.

22. Reinthaler FF, Posch J, Feierl G, Wüst G, Haas D, Ruckenbauer $G$, et al: Antibiotic resistance of E. coli in sewage and sludge. Water Res 2003; 37: 1685-1690.

23. Larsson DGJ, de Pedro C, Paxeus N: Effluent from drug manufactures contains extremely high levels of pharmaceuticals. J Hazard Mater 2007; 148: 751-755.

24. Ji X, Shen O, Liu F, Ma J, Xu G, Wang Y, et al: Antibiotic resistance gene abundances associated with antibiotics and heavy metals in animal manures and agricultural soils adjacent to feedlots in Shanghai; China. J Hazard Mater 2012; 235-236: 178-185.

25. Diwan V, Tamhankar AJ, Khandal RK, Sen S, Aggarwal M, Marothi Y, et al: Antibiotics and antibiotic resistant bacteria in waters associated with a hospital in Ujjain, India. BMC Public Health 2010; 10: 414.

26. Moreno MA: Survey of quantitative antimicrobial consumption per production stage in farrow-to-finish pig farms in Spain. Vet Rec Open 2014; 1:e000002.

27. Hermann-Bank ML, Skovgaard K, Stockmarr A, Lenz Strube $M$, Larsen $N$, Kongsted $H$, et al: Characterization of the bacterial gut microbiota of piglets suffering from new neonatal porcine diarrhoea. BMC Vet Res 2015; 11: 139.

28. Nhung NT, Chansiripornchai N, Carrique-Mas JJ: Antimicrobial resistance in bacterial poultry pathogens: a review. Front Vet Sci 2017; 4: 126.

29. Bäuerl C, Collado MC, Zúñiga M, Blas E, Pérez Martínez G: Changes in cecal microbiota and mucosal gene expression revealed new aspects of epizootic rabbit enteropathy. PLoS One 2014; 9: e105707.

30. Oliver SP, Murinda SE: Antimicrobial resistance of mastitis pathogens. Vet Clin North Am Food Anim Pract 2012; 28: 165-185.

31. Saini V, McClure JT, Scholl DT, DeVries TJ, Barkema HW: Herd-level association between antimicrobial use and antimicrobial resistance in bovine mastitis Staphylococcus aureus isolates on Canadian dairy farms. J Dairy Sci 2012; 95:1921-1929.

Ann Nutr Metab 2018;72 (suppl 1): 1-76 27 
32. Krömker V, Leimbach S: Mastitis treatment. Reduction in antibiotic usage in dairy cows. Reprod Domest Anim 2017; 52 Suppl 3: 21-29.

33. Crispie F, Alonso-Gómez M, O'Loughlin C, Klostermann K, Flynn J, Arkins S, et al:. Intramammary infusion of a live culture for treatment of bovine mastitis: effect of live lactococci on the mammary immune response. J Dairy Res 2008; 75: 374-384.

34. Frola ID, Pellegrino MS, Espeche MC, Giraudo JA, Nader-Macias ME, Bogni CI: Effects of intramammary inoculation of Lactobacillus perolens CRL1724 in lactating cows' udders. J Dairy Res 2012; 79: 84-92.

35. Qiao J, Kwok L, Zhang J, Gao P, Zheng Y, Guo Z, et al: Reduction of Lactobacillus in the milks of cows with subclinical mastitis. Benef Microbes 2015; 6:485-490.

36. Yu J, Ren Y, Xi X, Huang W, Zhang H: A novel lactobacilli-based teat disinfectant for improving bacterial communities in the milks of cow teats with subclinical mastitis. Front Microbiol 2017; 8: 1782.

37. APROMAR: La acuicultura en España. 2015. Documento disponible en www.apromar.es.

38. Cabello FC: Heavy use of prophylactic antibiotics in aquaculture: a growing problem for human and animal health and for the environment. Environ Microbiol 2006; 8: 1137-1144.

39. Grave K, Lingaas E, Bangen M., Rønning M: Surveillance of the overall consumption of antibacterial drugs in humans, domestic animals and farmed fish in Norway in 1992 and 1996. J Antimicrob Chemother 1999; 43: 243-252.

40. Sørum H: Antimicrobial drug resistance in fish pathogens. In Antimicrobial Resistance in Bacteria of Animal Origin. Aarestrup FM (editor), pp. 213-238. ASM Press, Washington DC, 2006.

41. Smith PR, Le Breton A, Horsberg TE, Corsin F: Guidelines for antimicrobial use in aquaculture. In: Guide to Antimicrobial Use in Animals. Guardabassi L, Jensen LB, Kruse H. (editors), pp. 207-218. Blackwell Publishing, Oxford, 2009.

42. Cabello FC, Godfrey HP, Tomova A, Ivanova L, Dölz H, Millanao A: Antimicrobial use in aquaculture re-examined: its relevance to antimicrobial resistance and to animal and human health. Environ Microbiol 2013; 15: 1917-1942.

43. Sørum H: Antibiotic resistance associated with veterinary drug use in fish farms. In: Improving Farmed Fish Quality and Safety. Lie, Ø. (ed.), pp. 157-182. Woodhead Publishing, Cambridge, 2008.

44 Samuelsen OB, Torsvik V, Ervik A: Longrange changes in oxytetracycline concentration and bacterial resistance toward oxytetracycline in a fish farm sediment after medication. Sci Total Environ 1992; 114: 125-126

45. Schmidt AS, Brunn MS, Dalsgaard I, Pedersen K, Larsen JL: Occurrence of antimicrobial resistance in fish-pathogenic and environmental bacteria associated with four Danish rainbow trout farms. Appl Environ Microbiol 2000; 66: 4908-4915.

46. Buschmann AH, Tomova A, Lopez A, Maldonado MA, Henriquez $L A$, Ivanova, $L$, et al: Salmon aquaculture and antimicrobial resistance in the marine environment. PLoS ONE 2012; 7: e42724.

47. Shah SQA, Cabello FC, L'Abée-Lund TM, Tomova A, Godfrey HP, Buschmann AH et al:. Antimicrobial resistance and antimicrobial resistance genes in marine bacteria from salmon aquaculture and non-aquaculture sites. Environ Microbiol 2014; 16, 1310-1320.

48. Lloyd DH: Reservoirs of antimicrobial resistance in pet animals. Clin Infect Dis 2007; 45 (Supplement 2): S148-S152.
49. Guardabassi L, Schwarz S, Lloyd DH: Pet animals as reservoirs of antimicrobial-resistant bacteria. J Antimicrob Chemother 2004; 54: 321-332.

50. Manian FA: Asymptomatic nasal carriage of mupirocin-resistant, methicillin-resistant Staphylococcus aureus (MRSA) in a pet dog associated with MRSA infection in household contacts. Clin Infect Dis 2003; 36: e26-e28.

51. Gandolfi-Decristophoris P, Regula G, Petrini O, Zinsstag J, Schelling E: Prevalence and risk factors for carriage of multi-drug resistantStaphylococciin healthy cats and dogs. J Vet Sci 2013; 14: 449.

52. WHO: Global action plan on antimicrobial resistance. WHO, Geneve, 2015.

53. WHO Critically important antimicrobials for human medicine publication, 5th revision. WHO, Geneve, 2017.

54. EMA: Antimicrobial resistance in veterinary medicine. http:// www.ema.europa.eu/ema/index.jsp?curl=pages/regulation/general/ general_content_001686.jsp\&mid=WC0b01ac05807a4e0d

55. Spellberg B, Hansen GR, Kar A, Cordova CD, Price LB, Johnson JR: Antibiotic resistances in humans and animals. National Academy of Medicine, 2016.

56. Xiao Y, Li L: China's national plan to combat antimicrobial resistance. Lancet Infect Dis 2016; 16: 1216-1218.

58. EIP-AGRI Focus Group on Reducing antibiotics in pig farming: Final report. 2014.

https://ec.europa.eu/eip/agriculture/en/publications/eip-agri-focus-group-reducing-antibiotics-pig

59. Sheehan E: Teagasc report: Actions taken to reduce antimicrobial usage on farm in Ireland. ReAct programme in The Netherlands, 2016.

60. FAO/WHO: Joint FAO/WHO working group report on drafting guidelines for the evaluation of probiotics in food. FAO, 2002.

61. Hill C, Guarner F, Reid G, Gibson GR, Merenstein DJ, Pot B, et al: Expert consensus document. The International Scientific Association for Probiotics and Prebiotics consensus statement on the scope and appropriate use of the term probiotic. Nat Rev Gastroenterol Hepatol 2014; 11: 506-514.

62. Rodríguez JM: Probióticos: del laboratorio al consumidor. Nutr Hosp 2015; 31(S1): 33-47.

63. Bakken JS, Borody T, Brandt LJ, Brill JV, Demarco DC, Franzos $M A$, et al: Treating Clostridium difficile infection with fecal microbiota transplantation. Clin Gastroenterol Hepatol 2011; 9: 1044-1049.

64. van Nood, E., Vrieze, A., Nieuwdorp, M., Fuentes, S., Zoetendal, E. G., de Vos, W. M., et al: Duodenal infusion of donor feces for recurrent Clostridium difficile. New Engl J Med 2013; 368: 407-415.

65. Nurmi EV, Rantala M: New aspects of Salmonella infection in broiler production. Nature 1973; 241: 210.

66. Stavric S: Defined cultures and prospects. Int J Food Microbiol 1992; 15: 245-263.

67. Nakamura A, Ota Y, Mizukami A, Ito T, Ngwai YB, Adachi $Y$. Evaluation of aviguard, a commercial competitive exclusion product for efficacy and after-effect on the antibody response of chicks to Salmonella. Poult Sci 2002; 81: 1653-1660.

68. Revolledo L, Ferreira CS, Ferreira AJ: Prevention of Salmonella Typhimurium colonization and organ invasion by combination treatment in broiler chicks. Poult Sci 2009; 88: 734-743.

69. Allen-Vercoe E, Reid G, Viner N, Gloor GB, Hota S, Kim P, et al: A Canadian Working Group report on fecal microbial therapy: microbial ecosystems therapeutics. Can J Gastroenterol 2012; 26: 457-462. 
70. Petrof EO, Gloor GB, Vanner SJ, Weese SJ, Carter D, Daigneault $M C$, et al: Stool substitute transplant therapy for the eradication of Clostridium difficile infection: 'RePOOPulating' the gut. Microbiome 2013; 1: 3.

71. Laycock G, Sait L, Inman C, Lewis M, Smidt H, van Diemen $P$, et al: A defined intestinal colonization microbiota for gnotobiotic pigs. Vet Immunol Immunopathol 2012; 149: 216-224.
72. DePeters EJ, George LW: Rumen transfaunation. Immunol Lett 2014; 162: 69-76.

73. Klieve AD, Hennessy D, Ouwerkerk D, Forster RJ, Mackie RI, Attwood GT: Establishing populations of Megasphaera elsdenii YE 34 and Butyrivibrio fibrisolvens YE 44 in the rumen of cattle fed high grain diets. J Appl Microbiol 2003; 95: 621-630.

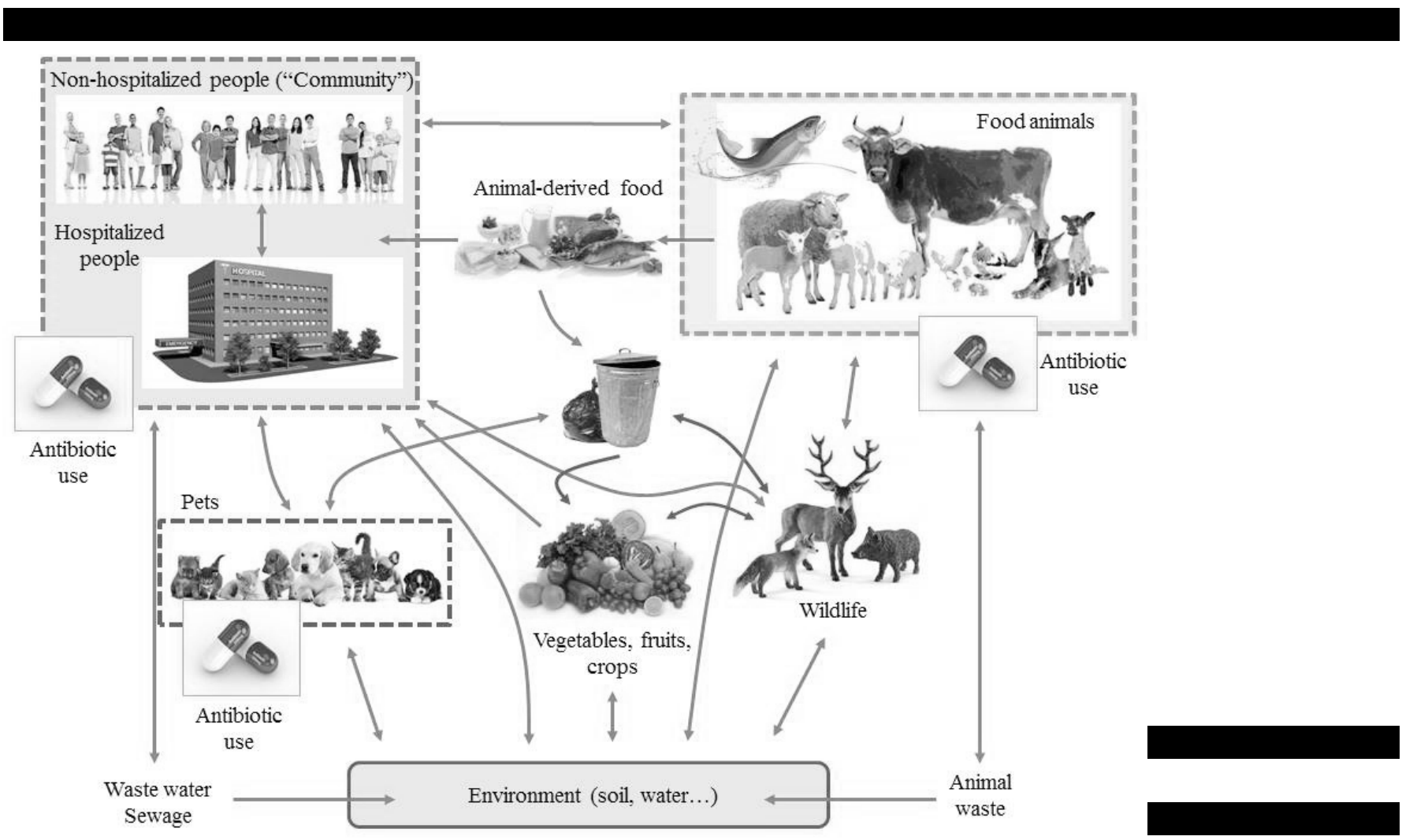

Figure 1. Antimicrobial resistance from animal and human origin. Ecology, epidemiology and relationships between potential sources of antibiotic-resistant microorganisms.

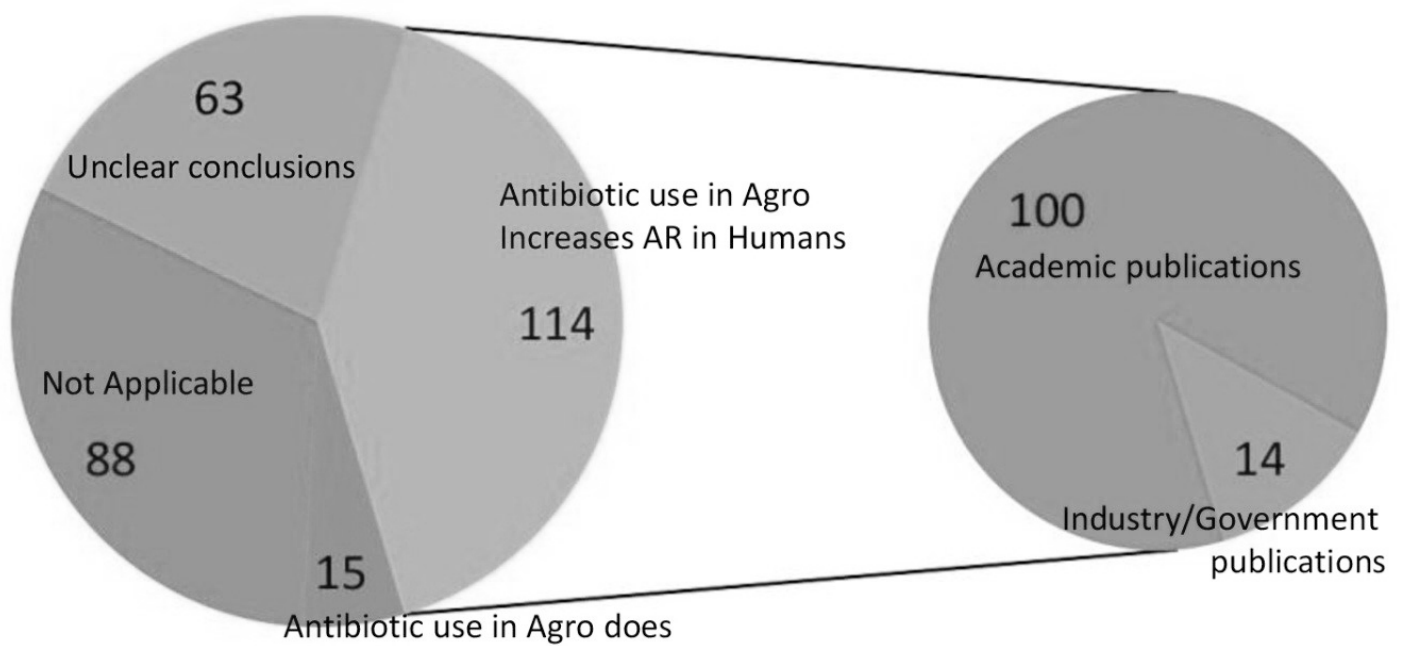

Figure 2. Proportions of 280 articles that relate antibiotic use in agriculture to the development of antibiotic resistance (AR) in humans. Source: [21]. 



\section{Nutritions of Metabolism}

\section{Track 2: Clinical Uses}

\section{PROBIOTICS, PREBIOTICS, AND SYNBIOTICS IN INFANT FORMULAE}

J.M. Moreno.

Paediatric Department Navarra Hospital Complex. Madrid. Spain.

The human colonic microbiota has a central role in health and disease. In addition to its nutritive role, human milk also guides the development of the intestinal microbiota. Human milk oligosaccharides, a major component in human milk, selectively stimulate the growth of Bifidobacteria and Lactobacillus. In the last few years several attempts have been made to obtain a similar microbiota in formula-fed infants. One way is to enhance the potential helpful microbiota adding selective nutrients, as is the case for prebiotics. Commercial oligosaccharides used in infant formula include galacto-oligosaccharides, polydextrose, inulin, and fructo-oligosaccharides. Their effects on infant growth, microbiota, stool characteristics, mineral absorption, and biomarkers of immune function have been assessed.

Prebiotics significantly increase the number of Bifidobacteria in a dose-related way and reduce pathogens. Some clinical studies have shown clinical benefits with the addition of prebiotics to infant formulas: decreased risk of atopic dermatitis and the incidence of intestinal and upper airway infections in the first two years of life and even beyond.

Recently, Human Milk Oligosaccharides (HMOs) have been technology produced and added to formulae, obtaining a microbiota profile more similar to that found in breast-fed infants. First clinical studies have shown that the addition of HMOs to infant formula is also associated with a decrease in upper respiratory infections as well as in diarrhoea.

In addition, human milk is a source of bacteria including staphylococci, streptococci, corynebacteria, lactic acid bacteria, propionibacteria, and bifidobacteria to the infant gut; however, the origin of milk bacteria, as well as their impact on neonatal gut microbiota establishment, remains largely unknown. In the past years, results provided by different research groups suggest that certain bacteria from the maternal gastrointestinal tract could translocate through a mechanism involving mononuclear immune cells, migrate to the mammary glands via an endogenous cellular route (the bacterial entero-mammary pathway), and subsequently colonize the gastrointestinal tract of the breast-fed neonate. When similar bacteria are added to infant formula -known as probiotics- they may produce an effect that mimics the one done by breast-milk bacteria.

Finally, some formulas add both prebiotics and probiotics, in different combinations and amounts, with the intention of taking advantage of the benefits of both nutrients. This combination is known as synbiotic.

\section{Track 3: Microbiology}

\section{‘OMICS' APPROACHES TO STUDY THE ORAL MI- CROBIOME}

\author{
A. Mira. \\ Leader of the Oral Microbiome Laboratory at the Center \\ for Advanced Research in Public Health (CSISP). FISABIO \\ Foundation. Valencia. Spain.
}

Bacteria inhabiting the human body play important roles for health that are still not fully understood. Those bacteria inhabit our gut, respiratory tract, skin, oral cavity, urinary tract, etc., and can be considered as an organ whose function needs to be elucidated. An important fraction of the human-associated microbiota has not been cultured and therefore, the use of high-throughput DNA, RNA and Protein techniques has revolutionized the research in the field. A basic technique that has been very successful is the massive sequencing of the $16 \mathrm{~S}$ rDNA gene. However, it only provides information about taxonomic composition and is subject to an important experimental bias. Metagenomics is the study of all genes present in the bacterial community obviating the need for culture and the recent development of second and third-generation sequencing techniques have allowed researchers to describe the composition and genetic repertoire of the human microbiome to an unprecedented level of detail. We have applied these techniques to describe the microbiota associated to the respiratory tract, the human breast milk, the stomach, the gut and specially the oral cavity, where we have identified a new bacterial species, named Streptococcus dentisani, that appears to protect against tooth decay. The direct RNA sequencing of oral samples has also allowed us to study the active organisms and the actively expressed genes at a given time point. This metatranscriptome approach has revealed important aspects about the etiology of dental caries and gum disease. In addition, the use of mass spectrometry in dental plaque samples has allowed us to describe the metaproteome of this oral biofilm in caries-free and caries-active individuals, identifying several biomarkers of disease. Finally, the combination of flow-cytometry and cell sorting with massive sequencing has served us to identify the bacteria recognized by different antibodies in the oral cavity, the gut and the breast milk. Thus, the combination of these techniques will serve to unravel the function of our microbial partners in health and disease. 


\section{METABOLOMICS: AN ESSENTIAL TOOL IN THE STUDY OF METABOLISM AND THE MICROBIOTA}

\author{
X. Correig. \\ Matabolomics Platform, Universitat Rovira I Virgili - CIBERDEM- \\ IISPV.
}

Metabolomics is the most recent and emerging omics science, which have revolutionized the study of the metabolism of live being and is being applied extensively in biomedical and clinical research. Nowadays, metabolomics is a necessary science in live sciences research, as an essential part in systems biology (1). In this talk I'll briefly introduce the main steps included in a metabolomics experiment, consisting in the experimental design, analytical measurement with massive platforms, signal processing, multivariate data analysis and biostatistics, metabolite identification and pathway analysis. The main applications of metabolomics in biomedicine will also be reviewed, emphasizing those related with the study of metabolism and metabolic diseases. In particular, I'll mention the role of metabolomics in the nutrition sciences, through the results got by the Metabolomics Platform (MP, http://metabolomicsplatform.com/) in several experiments.

The MP is a joint research facility created by URV (Universitat Rovira i Virgili, Tarragona, Spain) and CIBERDEM (Spanish Biomedical Research Network in Diabetes and Associated Metabolic Disorders). The main goal of the Metabolomics Platform is to offer metabolomic research collaborations to the biomedical and clinical research groups of CIBERDEM and URV.

The specific metabolic techniques used for the study of the microbiota and its contribution to the metabolism will be highlighted. In this talk I'll describe in detail some metabolomics techniques developed in the MP that are been used extensively in nutrition. We have particularly developed an advanced lipoprotein test, based on proton nuclear magnetic resonance spectroscopy (1H-NMR), able to quantify the particle number and size of 9 lipoprotein subfractions (2). This technique has been tested in more than 25.000 samples and 50 clinical studies and have proven it's efficacy in assessing dyslipidemia and cardiometabolic risk. It's now commercialized by Biosfer Teslab (http://biosferteslab.com/), a spin-off company of URV. We will also describe our recent advances in the metabolite profiling by $1 \mathrm{H}-\mathrm{NMR}$, paying attention to the results obtained with urine and faeces, two biological matrices that contain valuable information about microbiota composition and activity. I'll also review our most recent results in the profiling of lipids in serum extracts and its application in a clinical study about incorporation of long-chain n-3 polyunsaturated fatty acids into blood lipids (3).

(1) Systems Biology of Metabolism, Jens Nielsen, Annu. Rev. Biochem. 2017. 86:245-75

(2) Liposcale: a novel advanced lipoprotein test based on 2D diffusion-ordered $1 \mathrm{H}$ NMR

spectroscopy, R. Mallol et al. Journal of Lipid Research Volume 56, 2015 737-746

(3) LipSpin: a new bioinformatics tool for quantitative $1 \mathrm{H}-\mathrm{NMR}$ lipid profiling, R. Barrilero, et al. (Submetted to AC).

\section{Track 4: Veterinary}

\section{PROBIOTICS AS AN ALTERNATIVE TO ANTIBIOT- ICS USED IN ANIMAL PRODUCTION}

\author{
Sobrino Abuja, Odón Julián \\ Ministry of Agriculture and Fishing, Food and Environment. \\ Spain.
}

WHO and other international health agencies strongly advise that antimicrobial resistance is a global concern that could jeopardize people's future health.

Inappropriate use or misuse of antimicrobials is considered to be a major cause of antimicrobial resistance, both in human and animals. In some countries, the use of antimicrobials in animals is particularly relevant, and this situation needs to be urgently reversed.

In veterinary medicine, a close relationship has been found between the use of antimicrobials and the appearance of resistance. Those countries where the consumption of antimicrobials is highest tend to have high resistance profiles in pathogenic microorganisms, as evidenced by reports on consumption of critical antibiotics and the occurrence of resistance in microorganisms isolated from both clinical cases and carcasses in slaughterhouses.

Antimicrobial resistance control strategies include encouraging the development of new molecules to replace those antimicrobials that are already ineffective in the treatment of infectious processes. However, it is not expected that sufficient therapeutic tools will be available in the coming years to deal with the global threat we are facing.

It is therefore necessary to develop new strategies to preserve the antimicrobials currently available. The drastic limitations of antibiotic use in animals, especially in terms of prophylactic and metaphylactic use, could reverse the situation of resistance.

Antibiotic reduction plans mainly concern those critical antibiotics that are likely to be used in humans or those which, like colistine, constitute the reserve antibiotic for the treatment of infectious processes that are particularly resistant in humans. In addition, it is possible that other antimicrobials may be suppressed which, although they do not have a special effect on the development of resistance, they do have a special effect on the environment, such as zinc oxide. However, it is necessary to take the necessary measures so as not to endanger animal health and welfare and, furthermore, not to reduce animal production, which could jeopardise the food safety of the world's population.

There is an urgent need for changes in animal management, the introduction of new feeding and production techniques and, above all, a change in the focus of action on infectious processes, which are the main causes of antimicrobial use in animals.

The use of live microorganisms capable of modifying animal physiology or modulating the microbiota, both in the digestive tract and mucous membranes, is one of the most promising alternatives in the veterinary therapeutic field. The use of these micro-organisms in animal nutrition has so far been limited to their authorisation as zootechnical additives or silage agents.

9th Workshop SEPyP 
It is necessary to go one step further, so that they can constitute an effective, technically and economically viable alternative to the prophylactic and metaphylactic use of antimicrobials1, allowing the latter to be reserved for strictly therapeutic uses.

\section{MICROBIOTA AND BIOLOGICAL CONTROL STRAT- EGIES IN AQUACULTURE}

Pérez Sánchez, Tania

School of Veterinary Medicine. University of Zaragoza. Spain.

Aquaculture has become an increasingly important food source worldwide and experienced a remarkable development during the last years; however, infectious diseases have become a limiting factor for further intensification. Although antimicrobials have been commonly used as prophylactic and therapeutic agents, their use has led to the emergence of antimicrobial resistance. In this context, the search for alternative methods to control and prevent diseases is considered an essential environmentally-friendly tool. The conference will offer an update on the successes and challenges of biological approaches - probiotics, prebiotics, synbiotics, postbiotics, bacteriophages or plant-derived natural compounds (phytobiotics) - for disease prevention and control in aquaculture. Although most of these approaches are still in research and development stages, some of them have shown promising results in field trials. Therefore, a better understanding of the mechanisms of action of these approaches will help to maximize their beneficial properties.

\section{MICROBIOTA AND PROBIOTICS IN PIG PRODUC- TION.}

Susana María Martín Orúe

Animal Welfare and Nutrition Service. Animal and Food Science

Department. Autonomous University of Barcelona. Spain.

Probiotics have been used in the pig industry since some decades ago. Along the pig productive life there are different critical moments when probiotics can play a role. The post-natal and post-weaning periods are probably the most critical ones, when diarrhoea is frequent and when the initial intestinal colonization pattern and an adequate development of the immune system can be determinant for the future of the animal. The growing phase can also be a challenging period when animals should face changes of diet and environment that can compromise their performance. For breeding sows, the intensive reproductive cycles also represent a significant stress, having the sow a fundamental role transferring first bacteria to the newborn piglet and passive immune protection through milk.

Probiotic concept in animal production, and particularly in the pig industry, was initially conceived from a different perspective compared to human medicine. Whereas in humans, probiotic was always associated to improve health and to prevent or treat disease, in the feed industry the inclusion of probiotics was initially conceived as an alternative way to deliver antimicrobial compounds substituting antibiotics, or delivering enzymes to help the digestion of feed. However, in the recent times the increasing evidence

9th Workshop SEPyP for an essential role of the intestinal microbiota promoting health and welfare in farm animals has open new opportunities for the use of probiotics in pigs. Despite of this, the use of probiotics still is seen with skepticism by the industry as results are not always consistent and predictable.

In this presentation some recent evidences regarding the efficacy of probiotic strategies in particular applications will be reviewed. Possible uses to modulate the immune response and metabolism will be analysed in order to improve nutrient availability and feed efficiency, to prevent gastrointestinal disorders and to improve reproductive performance or to reduce stress. We will also discuss which could be the main limitations for an efficient use of probiotics in the field and those reasons that could be behind their inconsistency and unpredictability. We will also comment on the limited viability of some strains to be administered in the feed and possible alternatives. Finally, we will try to list those aspects that should be improved and considered in the development of probiotic strategies to move from a mostly empiric use to a more knowledge-based. 



\section{Nutritionals of Metabolism}

\section{Track 1: Immunonutrition}

\section{Reviewers:}

Ascensión Marcos Sánchez

Institute of Science and Technology of Food and NutritionICTANCSIC. Madrid.

Mónica de la Fuente del Rey

Biological Sciences Department. Universidad Complutense de Madrid.

Jose Manuel Martín Villa

School of Medicine. Universidad Complutense de Madrid.

$309 / 20$

\section{CARDIOVASCULAR PROTECTIVE EFFECTS OF BI- FIDOBACTERIUM BREVE CECT7263 AND SHORT- CHAIN FATTY ACIDS SUPPLEMENTATION IN MIN- ERALOCORTICOID-INDUCED HYPERTENSION}

\author{
M. Romero-Pérez; N. De La Visitación Pastor; I. Robles Vera; M. Toral \\ Jiménez; M. Sánchez Santos; R. Jiménez Moleón; F. Vargas; J. Duarte \\ Pérez. \\ Department of Pharmacology. School of Pharmacy. University of \\ Granada. Granada. Spain.
}

Introduction: Probiotics affect the composition and the diversity of the gut microbiota, and their potential role in different diseases is currently a hot topic for research. Recent evidence has shown that probiotics play an important role in hypertension, but the mechanisms involved have not been elucidated yet1. Mineralocorticoid-induced hypertension is associated with low gut acetate-producing bacteria2.

Objectives: The aim of this study was to investigate whether changes in gut microbiota induced by probiotic treatment or supplementation with the short-chain fatty acids (SCFAs) prevent cardiovascular changes on a renin-independent hypertension model induced by deoxycorticosterone acetate (DOCA)-salt.

Methods: We studied the effects of chronic treatment with the probiotic Bifidobacterium breve CECT7263 (BFM) or SCFAs, butyrate or acetate, on blood pressure, endothelial function, and oxidative status in DOCA-salt-induced hypertension. Animals were randomly divided into five groups: $(n=10)$ : control, DOCA-salt, treated DOCA-salt-BFM (109 CFU day-1 by oral gavage), treated DOCA-salt-butyrate $(0,5 \mathrm{mg} \mathrm{kg}-1$ day- 1$)$, treated DOCA-salt-acetate (10,7 mg kg-1 day-1) both in drinking water, for 5 weeks.
Results: We found that both BFM and acetate treatments prevented the increase in systolic blood pressure (SBP) and heart and kidney hypertrophy induced by DOCA-salt. Moreover, they also improved the impaired nitric oxide-dependent vasodilatation induced by acetylcholine in aortic rings from DOCA-salt and reduced the rise in aortic nicotinamide adenine dinucleotide phosphate (NADPH) oxidase activity found in DOCA-salt animals. None of these effects were observed in treated DOCA-salt-butyrate.

Conclusions: BFM and acetate which constitutes one of the main metabolites of the gut microbiota prevent hypertension, endothelial dysfunction and vascular oxidative stress by inhibition of NADPH oxidase activity.

References: 1. Robles-Vera et al. Antihypertensive Effects of Probiotics. Curr Hypertens Rep. 2017;19:26.

2. Marques et al. High-Fiber Diet and Acetate Supplementation Change the Gut Microbiota and Prevent the Development of Hypertension and Heart Failure in Hypertensive Mice. Circulation. 2017;135:964-77.

Conflict of interest: Authors declare no conflict of interest.

\section{$309 / 44$ \\ PRELIMINARY STUDY ABOUT THE RELATION- SHIP BETWEEN NON-EXTRACTABLE POLYPHE- NOLS FROM DIET, GUT MICROBIOTA AND OXI- DATIVE STRESS}

\footnotetext{
${ }^{(1)}$ I. Gutierrez Díaz; ${ }^{(1)}$ T. Fernández Navarro; ${ }^{(2)}$ J. Pérez Jiménez; ${ }^{(3)} N$. Salazar Garzo; ${ }^{(3)} C$. González De Los Reyes-Gavilán; ${ }^{(3)} M$. Gueimonde; ${ }^{(1)}$ S. González Solares.

(1)Department of Functional Biology. University of Oviedo. Oviedo. Spain. Group "Diet Microbiota and Health". Health Research Institute of Principality of Asturias (ISPA). Oviedo Spain.; ${ }^{(2)}$ Department of Metabolism and Nutrition. Institute of Food Science, Technology and Nutrition (ICTAN-CSIC). Madrid. Spain; ${ }^{(3)}$ Department of Microbiology and Biochemistry of Dairy Products. Dairy Research Institute of Asturias (IPLA-CSIC). Group Diet, Microbiota and Health. Health Research Institute of Principality of Asturias. (ISPA). Oviedo. Spain.
}

Introduction: Epidemiological studies have suggested an association between polyphenols intake and oxidative stress-related diseases. Information about the intake of polyphenols in human populations has been limited to the data available in nutrient databases, which do not include the food content of non-extractable polyphenols (NEPP). Recently, the information relative to these compounds in Spanish fruits and vegetables has been published ${ }^{1}$. 
Thus, since they are associated with the insoluble food matrix and may reach completely intact the colon, these compounds are of special interest in the study of diet-microbiota interactions.

Objectives: To analyse the intake of the total NEPP and their subclasses in a healthy adult population and to explore the relationship with the gut microbiota and oxidative stress biomarkers.

Methods: This study involved 103 healthy adults. Dietary intake was assessed by an annual food frequency questionnaire. NEPP content data were extracted from Pérez-Jimémez et al.1 and dietary fiber content data from Marlet et al. Serum malondialdehyde (MDA) levels were determined and the major phylogenetic microbial types present in the gut quantified by qPCR.

Results: NEPP represent a $73.64 \%$ of the total phenolic intake. From them, hydrolysable polyphenols, and non-extractable flavonols, hydroxybenzoic and hydroxycinnamic acids, were negatively associated with MDA. Non-extractable hydroxybenzoic acid was found to be an independent contributor to Bacteroides-Prevotella-Porphyromonas group levels ( $\mathrm{R} 2=0.053, \beta=0.230)$, whereas Clostridium cluster XVIa variation was explained by the intake of non-extractable flavonols and Klason lignin (R2=0.150, $\beta=-$ 0.318 and 0.297 , respectively). Moreover, the levels of Lactobacillus group were associated with non-extractable flavonols intake $(\mathrm{R} 2=0.095, \beta=-0.308)$.

Conclusions: NEPP are associated with some of the major intestinal microbial groups and oxidative stress. Our data could be of great interest for future studies in order to elucidate the complex relation among diet-microbiota-health.

Reference: Pérez-Jiménez, J. et al. Food Res. Int. 74, 315-323 (2015)

Conflict of interest: Authors declare no conflict of interest.

\section{$309 / 50$ \\ EFFECT OF YOGURT AND PROBIOTIC FERMENT- ED MILK CONSUMPTION IN THE GUT MICROBI- OTA OF HEALTHY ADULTS}

\section{N. Redondo Useros; A. Gheorghe; L. Diaz; B. Villavisencio Seminario; A. Marcos; E. Nova Rebato.}

Immunonutrition Group. Metabolism and Nutrition Department. Institute of Food Science. Technology and Nutrition. Spanish

National Research Council. Madrid. Spain.

Introduction: Yogurt consumption has been related to beneficial effects on gastrointestinal (GI) health, such as maintenance of a healthy gut microbiota and prevention of some gastrointestinal diseases1, although studies in healthy adults are inconclusive.

Objectives: To assess the influence of yogurt and probiotic fermented milk (PFM) consumption on gut microbiota composition at the phylum level in healthy adults.

Methods: 261 adults aged between $25-45$ y, not suffering from any chronic disease or following medical treatment were included (51\% males). Gut microbiota composition was analyzed in fecal samples through 16S rRNA gene amplicon sequencing (V3+V4 gene regions. MiSeq 2x250 Illumina) and taxonomic analysis. Yogurt and PFM consumption habits were analyzed in a weekly basis with a food frequency questionnaire, and the following groups were considered: Non-consumers (0 y/week), Low (>0-2 y/week), Medium (3-4 y/week) and High ( $\geq 5 \mathrm{y} /$ week). PFM consumption was quantified by a specific questionnaire including Lactobacillus and Bifidobacterium-enriched products.

Results: There were no differences among yogurt consumption groups. However, PFM consumers showed higher Actinobacteria $(\mathrm{P}=0.011)$ and lower Proteobacteria levels $(\mathrm{P}=0.021)$ compared to non-consumers. A trend for a lineal relationship was observed between PFM products consumed per week and Actinobacteria levels $(\mathrm{P}=0.071)$. Gender differences were observed regarding PFM - microbiota interactions. Indeed, male consumers showed higher Firmicutes $(\mathrm{P}=0.052)$ and lower Bacteroidetes levels $(\mathrm{P}=0.057)$ compared to non-consumers, but no differences were observed in females.

Conclusions: PFM consumption might have a positive influence on the microbiota, since numerous Actinobacteria members have been related to beneficial effects on GI health2. The gender differences in the probiotic-microbiota interactions suggest that gender should be considered when planning to use probiotic therapy3.

Reference: Adolfsson O et al. (2004) Yogurt and gut function. Am J Clin Nutr. 80:245-56.

Suzuki Y et al (2017) Association between Yogurt Consumption and Intestinal Microbiota in Healthy Young Adults Differs by Host Gender. Front Microbiol. 8:847. doi: 10.3389/fmicb.2017.00847

Bäckhed F et al. (2012) Defining a healthy human gut microbiome: current concepts, future directions, and clinical applications. Cell Host Microbe. 12(5):611-22. doi: 10.1016/j.chom.2012.10.012

Conflict of interest: Authors declare no conflict of interest.

\section{$309 / 66$}

\section{LACTOBACILLUS RHAMNOSUS GG IMPROVES BEHAVIOR AND IMMUNE FUNCTION IN PREMA- TURELY AGING MICE}

\author{
${ }^{(1)}$ P. Hernández; ${ }^{(2)}$ B. Arauzo; ${ }^{(1)}$ N. Ceprian; ${ }^{(1)}$ C. Hunsche; ${ }^{(2)}$ C. Pelaez; \\ ${ }^{(2)}$ C. Martínez-Cuesta; ${ }^{(2)}$ T. Requena; ${ }^{(3)} M$. De la Fuente. \\ (1)Department of Physiology. Faculty of Biology. Complutense \\ University of Madrid. Madrid. Spain.; ${ }^{(2)}$ Department of Food \\ Biotechnology and Microbiology. Institute of Food Science \\ Research (CIAL-CSIC). Madrid. Spain.; ${ }^{(3)}$ Institute of Investigation \\ Hospital 12 de Octubre (IIS i+12). Madrid. Spain.
}

Introduction: Ageing is characterized by an impairment of the homeostatic systems (nervous and immune systems) and consequently by a higher risk of morbidity and mortality. $\mathrm{Nu}$ tritional intervention is one of the most used strategies to slow down this deterioration, and recently the supplementation with probiotics has been suggested as a possible way of improving these systems. 9th Workshop SEPyP 
Objectives: This study evaluated the effects of the supplementation with Lactobacillus rhamnosus GG (LGG) on several behavioral and immune function parameters in a model of prematurely aging mice (PAM).

Methods: Adult PAM and non-prematurely aging mice (NPAM), were divided into two groups, which received LGG (109 CFU/mice/day; lyophilized in skim milk and added to drink water) or skim milk without LGG (controls). After 2 weeks, mice were submitted to behavioural tests (anxiety-like behaviours and exploratory capacity). After this, and after an extra 2 week LGG supplementation, peritoneal leukocytes were collected and several immune functions (macrophage and lymphocyte chemotaxis, macrophage phagocytosis, natural killer cell activity and lymphoproliferative response to mitogens) were analyzed.

Results: The higher anxiety levels together with the lower exploratory activity observed in PAM compared with NPAM disappeared after LGG supplementation. Similar results were shown with PAM premature immunosenescence, and after 2 and 4 weeks of LGG intake an improvement of leukocyte functions was observed, with similar values to those found in NPAM. LGG counts reached 6 log DNA copy/g faeces in PAM and NPAM. However, there were no differences between the faecal microbiota composition and metabolism of both groups (PAM and NPAM) at the starting of the assay and throughout 4 week LGG supplementation.

Conclusions: In conclusion, LGG ingestion improves nervous and immune functions in PAM. Since the parameters evaluated are health and ageing rate markers LGG could be useful for achieving a healthy longevity.

This work was supported by FIS (PI15/01787) of Health Institute Carlos III - Regional Developing Aid European Funds (ISCIII-FEDER), MINEICO (AGL2016-75951-R, CDTI (ADAPTEA IDI-20150753) and CYTED (P916PTE0233).

References: This work was supported by FIS (PI15/01787) of Health Institute Carlos III - Regional Developing Aid European Funds (ISCIII-FEDER), MINEICO (AGL2016-75951-R, CDTI (ADAPTEA IDI-20150753) and CYTED (P916PTE0233).

Conflict of interest: Authors declare no conflict of interest.

\section{9/70 \\ LACTOBACILLUS FERMENTUM ACTS AS A CAR- RIER OF IRON OXIDE NANOPARTICLES: A NEW STRATEGY FOR IRON SUPPLEMENTATION}

\footnotetext{
${ }^{(1)}$ A. Rodríguez-Nogales; ${ }^{(2)}$ V. Garcés; ${ }^{(2)}$ A. González; ${ }^{(2)} N$. Gálvez; ${ }^{(3)} M$. García; ${ }^{(4)}$ L. Gutiérrez; ${ }^{(5)}$ M. Olivares; ${ }^{(6)}$ J. Gálvez; ${ }^{(2)}$ J. Dominguez-Vera.

${ }^{(1)}$ Hospital Civil. Instituto de Investigación Biomédica de Málaga. Málaga.; ${ }^{(2)}$ Department of Inorganic Chemistry. Faculty of Science. University of Granada. Granada. Spain.; ${ }^{(3)}$ BIONAND. The Andalusia Technology Park. Málaga. Spain.; ${ }^{(4)}$ Institute of Nanoscience of Aragón. University of Zaragoza. Zaragoza. Spain.; ${ }^{(5)}$ Research Department of Biosearch. Granada. Spain.; ${ }^{(6)}$ Department of Pharmacology. CIBER-EHD. ibs. Granada. CIBM. University of Granada. Granada. Spain.
}

9th Workshop SEPyP
Introduction: Iron deficiency anaemia (IDA) is a nutritional-deficiency disease, especially in children and pregnant women, which may lead to morbidity and death. The treatment of IDA implies the increase of daily iron intake with supplements based on different chemical iron; however, their use is limited due to gastrointestinal side effects and low bioavailability.

Objectives: To investigate the effects of small maghemite nanoparticles (MNP) incorporated on Lactobacillus fermentum CECT5716 (MNP-probiotic) as iron supplement in rats with IDA.

Methods: MNP-probiotic was prepared following a previously described procedure. Male Wistar rats (180-200 g) were fed an iron deficient diet until the anaemia was established. Then, rats were divided in different experimental groups $(n=10)$ : Two groups received an iron supplement by MNP or MNP-probiotic suspensions; one untreated control group; one treated with L. fermentum, and other treated with iron sulphate. The dose of iron was in all cases $1 \mathrm{mg}$ per day per animal, and treated for 23 days. Then, the anaemic status was evaluated biochemically in blood and target tissues (intestine and liver).

Results: IDA was characterized by reduced haemoglobin levels in the untreated control group, which were reversed after treatment with iron sulphate or MNP-probiotic. In addition, in the MNP-probiotic this beneficial effect was associated with a restoration in mRNA expression of both DcytB (a ferrireductase protein) and DTM1 (Fe transporter) in the intestine. Moreover, the reduced expression of hepcidin in the liver from anaemic rats was significantly increased in those groups treated with iron sulphate or MNP-probiotic.

Conclusions: MNP-probiotic showed positive effects in rats under IDA, showing even a better profile than that found with iron sulphate, the standard pharmacological IDA treatment.

Reference: Martín M., et al. Magnetic study on biodistribution and biodegradation of oral magnetic nanostructures in the rat gastrointestinal tract. Nanoscale. 2016;8(32):15041-7.

Conflict of interest: Authors declare no conflict of interest.

\section{$309 / 75$ \\ ENDOCYTOSIS CAN BE A NEW CONCEPT THAT EXTENDS PARADIGMS OF PROBIOTIC/HOST IN- TERACTION}

\author{
${ }^{(1)}$ D. Ramírez Sánchez; ${ }^{(2)}$ C. Bäuerl; (2) J. Coll Marqués; ${ }^{(1)}$ S. Campista \\ Léon; ${ }^{(2)} G$. Pérez Martínez. \\ (1)Biology School. Autonomous University of Sinaloa. Calzada \\ de las Américas y Universitarios. Culiacán Rosales. México; \\ ${ }^{(2)}$ Departament of Biotechnology. Institute of Agrochemistry and \\ Food Technology Institute (IATA-CSIC). Valencia. Spain.
}

Introduction: Bacterial internalization into mucosal cells is part of the natural interaction of the intestinal microbiota with the host necessary to keep homeostasis. Pathogen phagocytosis by macrophages and capture of luminal bacteria by dendritic and epithelial cells have been thoroughly studied. Highly adherent lac-

Ann Nutr Metab 2018;72 (suppl 1): 1-76 
tic acid bacteria can transfect DNA into Caco2 cells ${ }^{1}$, but little is known about endocytosis and its role in the real interaction of between probiotics and host cells.

Objectives: This work aims to characterise the mechanism of internalisation of the probiotics Lactobacillus casei BL23 y Lactobacillus rhamnosus GG into epithelial cells by endocytosis (or autophagy).

Methods: For quantification of the internalisation into Caco2 and T84 cells the gentamicin protection method2, as well as microcopy and flow cytometry (FC) quantification were used. To determine what type of internalisation is occurring inhibitors like cytochalasin D and 5-(N-ethyl-N-isopropyl)-amiloride (EIPA) were used. EGF, IFN- $\gamma$ and rapamycin were used to determine the involvement of signal transduction pathways (cell proliferation, mTOR, etc.).

Results: Internalisation rates varied with the probiotic and the cell type. Cytochalasin D inhibited BL23 and LGG internalisation confirmed by FC, and EIPA specifically inhibited BL23 entry in Caco2, which did not agree with FC data. EGF increased internalisation. By FC, rapamycin seems to increase probiotic/cell binding. Epifluorescence showed that whole bacteria remain inside the cells after 24 hours and that IFN- $\gamma$ increases lysosome activity.

Conclusions: Early stages of internalisation have been analysed. Probiotics BL23 and LGG are internalised by actin dependent mechanisms although typical endocytosis structures were not observed. This process could have great relevance in the probiotic stimulation of epithelial cells and possibly other mucosal cells.

\section{References:}

1. Guimarães, et al . Genetic Vaccines and Therapy 2009;7:4-.

2. Elsinghorst. Methods in Enzymology: Academic Press, 1994:405-20.

Conflict of interest: Authors declare no conflict of interest.

\section{Track 2: Clinical Use}

\section{Reviewers:}

Francisco Guarner Aguilar

Departament of Gastroenterology. Hospital Vall d'Hebron.

Barcelona.

Guillermo Álvarez Calatayud

Department of Pediatrics. Gregorio Marañón Hospital. Madrid.

\section{Fernando Azpiroz Vidaur}

Departament of Gastroenterology. Hospital Vall d'Hebron.

Barcelona.

\section{Rosaura Leis Trabazo}

Paediatrics Professor. University of Santiago de Compostela. Gastroenterology, Hepatology and Paediatrics Nutrition Unit Coordinator at the University Hospital of Santiago.

\section{9/14 \\ KYO-DOPHILUS ${ }^{\circledR}$ PLUS ENZYMES: POTENTIAL APPLICATION IN LACTASE NON-PERSISTENCE}

\author{
${ }^{(1)}$ M. Villamiel Guerra; ${ }^{(2)}$ A. Ferreira-lazarte; ${ }^{(1)}$ F. Moreno Andújar; ${ }^{(3)}$ E. \\ Cano; ${ }^{(3)}$ A. Pellejero. \\ ${ }^{(1)}$ Department of Bioactivity and Food Analysis. Research \\ Institute of Food Science. CIAL (CSIC-UAM). Autonomous \\ University of Madrid. Madrid. Spain.; ${ }^{(2)}$ Institute of Food Science \\ Research. CIAL (CSIC-UAM). Madrid. Spain.; ${ }^{(3)}$ Vitae Natural \\ Nutrition S.L. Barcelona. Spain.
}

Introduction: Lactase non-persistence (LNP) is a case of primary hypolactasia, related to ethnicity, with a prevalence of $75 \%$ of world adult population due to a genetically-programmed reduction in $\beta$-galactosidase activity. When lactase is not stimulated by continued lactose ingestion, excess of undigested lactose osmotically attracts liquid into the gut lumen leading to diarrhoea. Moreover, unabsorbed lactose reaches the colon where is metabolised by the microbiota giving rise to short-chain fatty acids, $\mathrm{CO}_{2}, \mathrm{CH}_{4}$ and $\mathrm{H}_{2}$ causing abdominal discomfort. The strategies to avoid this inconvenience are mainly focused on the restriction of milk and dairy products and the intake of lactose-depleted and lactose-free products, non-exempt of nutritional and/or technical problems. The therapy with non-human $\beta$-galactosidases or probiotics endowed with $\beta$-galactosidase activity added at mealtime is other interesting approaches without side effects ${ }^{1}$.

Objectives: The aim of this work has been the evaluation of lactase activity of the commercial preparation Kyo-Dophilus ${ }^{\circledast}$ plus enzymes and its potential application in lactase non-persistence management.

Methods: This digestive support contains human strains of Lactobacillus gasseri KS-13, Bifidobacterium bifidum G9-1, Bifidobacterium longum MM-2 and protease, lactase, lipase and amylase from Aspergillus sp. 
Results: The obtained results underpins that the recommended amounts (460-1000 mg) were adequate for in vitro lactose hydrolysis $(0.25-5 \%)$ in lactose solutions and in milks and yogurts, with hydrolysis values in the range $44-96 \%$, depending on the enzymatic preparation/lactose ratio (w/w). Moreover, due to the transgalactosylation power of $\beta$-galactosidases present in this preparation, prebiotic galactooligosaccharides were also formed, increasing its potential benefits in the gastrointestinal system.

Conclusions: Taken into account the obtained percentages, the intake of lactose would be in agreement with the EFSA recommendations for individuals with lactase non-persistence diagnosis.

References: Bayless et al. Curr Gastroenterol Rep 2017, 19. [2] EFSA J 2010; 81777.

Conflict of interest: Authors declare no conflict of interest.

\section{$309 / 26$ \\ EFFECT OF A PREBIOTIC FORMULATION CON- TAINING INULIN AND FRUCTOOLIGOSACCHA- RIDES ON FRAILTY SYNDROME:A RANDOMIZED, DOUBLE-BLIND, PLACEBO CONTROLLED CLINI- CAL TRIAL}

\author{
${ }^{(1)}$ O. Cauli; (1)J. Fernández-garrido; ${ }^{(1)}$ C. Buigues; ${ }^{(1)}$ R. Navarro- \\ martínez; ${ }^{(2)}$ L. Pruimboom; ${ }^{(3)}$ A. Hoogland; ${ }^{(4)}$ M. Martínez Martínez; \\ ${ }^{(4)} Y$. Verdejo. \\ (1)Department of nursing. University of Valencia. Valencia. \\ Spain.; ${ }^{(2)}$ Natura Foundation. University of Groningen. University \\ Medical Center Groningen (umcg). Groningen. Netherlands.; \\ ${ }^{(3)}$ Bonusan Besloten Vennootschap. Numansdorp. Netherlands.; \\ ${ }^{(4)}$ Geroresidencias la Saleta. Valencia.
}

Introduction: Aging can result in major changes in the composition and metabolism of intestinal flora and results in impaired function of the immune system

Objectives: We assessed the efficacy of administration of prebiotic mixture Darmocare Pre $\left({ }^{\oplus}\right)$ containing inulin and fructooligosaccharides to evaluate whether the regular intake of this product can improve frailty criteria in elderly people affected by the frailty syndrome.

Methods: The study was a placebo-controlled, randomized, double blind design in sixty older participants aged 65 and over. Participants were randomized to a parallel group intervention of 13 weeks' duration with a daily intake of Darmocare Pre $\left({ }^{\circledast}\right)$ or placebo (maltodextrin). The primary outcome was to study the effect on frailty syndrome.

Results: The overall rate of frailty was not significantly modified by Darmocare Pre $\left({ }^{\circledast}\right)$ administration. Nevertheless, prebiotic administration compared with placebo significantly improved two frailty criteria, e.g., exhaustion and handgrip strength $(\mathrm{p}<0.01$ and $\mathrm{p}<0.05$, respectively).

Conclusions: The use of novel therapeutic approaches influencing the gut microbiota-muscle-brain axis could be considered

9th Workshop SEPyP for treatment of some of the symptoms of frailty syndrome in older individuals.

References: Buigues C et al. Int J Mol Sci. 2016 Jun 14;17(6).

Conflict of interest: Aldert J. Hoogland is a product developer in the company (Bonusan) that provided the Darmocare Pre ${ }^{\oplus}$ and the placebo for the study, and he performed the blinded randomization. Leo Pruimboom is a regular teacher in courses and seminars organized by Bonusan. Aldert J. Hoogland and Leo Pruimboom never

saw any of the participants or saw any of the results in this study. Cristina Buigues and Julio Fernández-Garrido wrote the manuscript. Omar Cauli and Leo Pruimboom reviewed and corrected the manuscript. The remaining authors (including Omar Cauli) declare that they have no competing interests, and they were blind to the drug treatment until the completion of the study.

\section{$309 / 34$ \\ COLLECTION DEVICE FOR KEEPING MICROBIO- TA VIABILITY}

${ }^{(1)}$ N. Martínez Álvarez; ${ }^{(2)}$ C. Hidalgo; ${ }^{(3)}$ A. Margolles; ${ }^{(4)}$ S. Delgado; ${ }^{(5)} B$. Sanchez.

(1)Responsable de i+d. Microviable therapeutics. Asturias. Spain.; ${ }^{(2)}$ Mcroviable therapeutics. Asturias. North Carolina State University. Raleigh. North Carolina. EEUU.; ${ }^{(3)}$ Department of Microbiology and Biochemistry of Dairy Products. Instituto de Productos Lácteos de Asturias (IPLA-CSIC). Group Diet, Microbiota and Health. Instituto de Investigación Sanitaria del Principado de Asturias (ISPA). Oviedo. Spain.; ${ }^{\left({ }^{4}\right)}$ Department of Microbiology and Biochemistry of Dairy Products. Dairy Research Institute of Asturias. Spanish National Research Council (IPLA-CSIC). Villaviciosa. Asturias. Spain.; ${ }^{(5)}$ Microviable therapeutics. IPLA-CSIC. Asturias. Spain.

Introduction: New studies and therapies based on gut microbiota sampling need from protocol normalization and standardization. During this process, keeping microbial viability from the sampling to the delivery, and the manipulation and storage of the faecal samples is crucial. In Microviable Therapeutics (MT) we have detected that this standardization requires a device/kit to avoid the toxic effect of oxygen on intestinal microbiota and we have developed a technological solution to fill this gap.

Objectives: To develop and validate an anaerobic stool sample collection device (PCT/ES2017/070087).

Methods: The efficiency of the device for anaerobic stool sampling was tested and compared with other stool collection systems. Maintenance of the anaerobic conditions was experimentally evaluated by a colorimetric assay and by means of total counts of anaerobic microorganisms. For this purpose, autoclaved faecal samples were inoculated with cultures of different anaerobic microorganisms. Recounts were carried out at inoculation time and after 24/72 $\mathrm{h}$ incubation at room temperature (RT).

Results: In the developed device, anaerobic microorganisms inoculated in sterile faecal samples showed a lower loss of viability after 24 and $72 \mathrm{~h}$ compared with conventional systems. Bifidobacte- 
rium bifidum remained viable and culturable up to $72 \mathrm{~h}$ at $\mathrm{RT}$, while using a conventional stool collection system the bifidobacterial population was reduced $58 \%$ at $24 \mathrm{~h}$ and more than $98 \%$ at $72 \mathrm{~h}$.

Conclusions: The anaerobic sample collection system developed ensures an anoxic environment for the stool sampling and transport until processing at the laboratory. As derived from the present data, bacterial viability is kept by a 100 -fold factor using this device. This could be critical in some applications, such as standardization and normalization of microbiota-based studies and the isolation of new probiotic strains from faeces.

References: Cammarota et al. 2017. GUT. doi: 10.1136/gutjnl-2016-313017

Conflict of interest: No competing interest exits.

\section{$309 / 36$ \\ GUT HEALTH IMPROVEMENT MEDIATED BY A SYNBIOTIC CONTAINING BIFIDOBACTERIUM LONGUM SUBSP. INFANTIS, LACTOBACILLUS RHAMNOSUS AND A PREBIOTIC MIXTURE OF INULIN: FRUCTOOLIGOSACHARIDES, IN A NEO- NATAL RAT MOD}

\author{
(1)J. Moreno Muñoz; ${ }^{(2)}$ A. Caimari; ${ }^{(2)} N$. Boqué; ${ }^{(2)}$ A. Crescenti; ${ }^{(2)} J$. \\ Teichenné; (2) J. Del Bas; ${ }^{(3)} G$. Cifuentes Orjuela; ${ }^{(3)} M$. Rodríguez \\ Palmero; ${ }^{(3)}$ J. Jiménez; ${ }^{(2)}$ L. Arola. \\ (1)Departament of Basic Research. ORDESA Laboratories. \\ Barcelona. Spain.; ${ }^{(2)}$ Eurecat-technology centre of Catalonia. \\ Nutrition and health unit. Tarragona. Spain.; ${ }^{(3)}$ Ordesa S.L. \\ Laboratory. Barcelona. Spain.
}

Introduction: Synbiotics were proposed for supplementation of infant foods as functional ingredients, but scientific data about their health effects is limited.

Objectives: An in vivo experiment was conducted using a neonatology rat model to study the effects in gut health of a synbyotic consisting in a mixture of Bifidobacterium longum subsp. infantis IM-1 and Lactobacillus rhamnosus HN001 with a prebiotic mixture of inulin:fructooligosacharides. The effects of the synbiotic were compared to those produced by the supplementation with a vehicle or with each of these bioactive mixtures.

Methods: Body weight, serum, food intake and depositions (frequency, weight and water content) were monitored throughout the study. Analyses of animal body composition by Proton Nuclear Magnetic Resonance (NMR) were also conducted during the study.

Results: Synbiotic-supplemented animals showed a significant decrease in relative weight of epididymal white adipose tissue (19\%) and mesenteric white adipose tissue (15\%), although no differences were observed in total fat mass content by NMR between synbiotic and control group. Supplementation with synbiotic potentiated the effects of probiotic mixture treatment on different parameters related to digestive health, obtaining, with respect to control group, significant increases of similar magnitude to those observed in the animals of the prebiotic mixture group in the weight of the caecum, the length of the colon, fresh weight of the faeces and their water content.

Probiotic group showed significant decrease in circulating levels of LPS and a significant increase in the ileal expression of the genes coding for mucus MUC2, Occludin and ZO2.

Conclusions: Bifidobacterium longum subsp. infantis IM-1, Lactobacillus rhamnosus HN001 and the prebiotic mixture (inulin:fructooligosacharides) is a promising synbiotic that could improve gut health in babies.

References: Clinical Nutrition 2013; 32 (6): 918-927

Obesity 2014; 22 (3): 763-771

Conflict of interest: Gloria Cifuentes-Orjuela, Maria Rodríguez-Palmero, J. Jiménez and J. A. Moreno Muñoz are employees of Laboratorios Ordesa.

\section{$309 / 85$}

\section{A RANDOMIZED CLINICAL TRIAL TO EVALUATE THE SAFETY AND THE EFFECT OF A PROBIOT- IC MIXTURE ON PASI INDEX IN PATIENTS WITH MODERATE PSORIASIS}

\author{
(1)V. Navarro-López; ${ }^{(2)}$ B. Ruzafa-Costas; ${ }^{(3)}$ E. Chenoll-Cuadros; \\ ${ }^{(4)}$ D. Ramon-Vidal; ${ }^{(3)}$ S. Genoves-Martinez; ${ }^{(2)} Y$. Payá-Espinosa; ${ }^{(5)} \mathrm{F}$. \\ Codoñer-Cortes; ${ }^{(2)}$ M. Carrion-Gutierrez. \\ ${ }^{(1)}$ Clinical Microbiology and Infectious Disease Unit. Hospital \\ Universitario Vinalopo. Elche. Department of Clinical Medicine. \\ MiBioPath Group. Universidad Catolica San Antonio de Murcia. \\ Murcia. Spain.; ${ }^{(2)}$ Department of Clinical Medicine. MiBioPath \\ Group. Universidad Catolica San Antonio de Murcia. Murcia. \\ Spain.; ${ }^{(3)}$ Biopolis S.L. Paterna. Valencia. Spain.; ${ }^{\left({ }^{(4)} B i o p o l i s ~ S . L . ~\right.}$ \\ Lifesequencing S. L. Paterna. Valencia. Spain.; ${ }^{(5)}$ Lifesequencing S. \\ L. Paterna. Valencia. Spain.
}

Introduction: In this clinical trial we hypothesized that the use of probiotic may play a role in the outcome of psoriasis.

Objectives: To determine the response to treatment, defined as an improving of the PASI index higher of $75 \%$. Secondary objectives: changes in the PGA index, inflammatory biomarkers in blood and side effects

Methods: From September 2015 to October 2016 ninety patients with psoriasis were randomized to receive probiotic vs placebo during 12 weeks. Blood count, biochemical and biomarkers (TNF- $\alpha$, IF- $\gamma$, IL-1b, IL-6, IL-12, IL-23) were measured. Gut microbiome analysis of stool samples were carried out.

Results: In the intermediate evaluation at week six, 15 of 45 (33.3\%)p. in the probiotic group and 5 of $43(11.4 \%)$ in the placebo group $(\mathrm{p}=0.016)$ reach a PASI75 response. At the end of the 12 weeks intervention period this differences increase to 30 of $45(66.7 \%)$ Vs 19 of 43 (44.9\%); $\mathrm{p}=0.013$. Difference was also 9th Workshop SEPyP 
observed in the blood levels of TNF when comparing the group of patients with PASI75 response (decrease of 2,33 $\pm 16,7$ ) and the

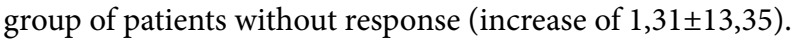

At the end of the 12 weeks framework (after stop treatment), patients were evaluated in to assess the occurrence of relapse in a period of 24 weeks outcome. Data shows a lower incidence of flares in the probiotic group: 9 of $45 p$. (20.0\%) Vs 18 of 43 (41.9\%); $p=0.01$

Conclusions: The results of our study indicate a strong positive effect in the group treated with the probiotic. It is also important that the number of new outbreaks after stopping treatment is lower in the probiotic group.

\section{References:}

1. Schäfer T. Epidemiology of psoriasis. Review and... Dermatology 2006; 212:327-37.

2. Ana Ramírez-Boscá, Vicente Navarro-López, Asunción Martínez-Andrés et al. Identification of bacterial DNA in... JAMA Dermatol. 2015 Jun;151:670-1

Conflict of interest: Non conflicts of interest disclosed

\section{Track 3: Microbiology}

\author{
Reviewers: \\ Abelardo Margolles Barro \\ Institute of Dairy Products of Asturias, IPLA-CSIC. Oviedo.
}

Alfonso Clemente Gimeno

Experimental Station of Zaidín (EEZ-CSIC). Granada.

Juan Evaristo Suárez Fernández

Oviedo University. Asturias.

Teresa Requena Rolanía

Research Institute in Food Sciences, CIAL (CSIC- UAM). Madrid.

Juan Miguel Rodríguez Gómez

Veterinary School. Universidad Complutense de Madrid.

Gaspar Pérez Martínez

Institute of Agrochemistry and Food Technology, IATA-CSIC.

Valencia.

\section{$309 / 8$}

\section{NON-IGE MEDIATED COW'S MILK PROTEIN AL- LERGY IN INFANCY}

\author{
${ }^{(1)}$ S. Delgado; ${ }^{(1)} M$. Díaz; ${ }^{(2)}$ S. Jiménez; ${ }^{(3)} \mathrm{C}$. Molinos; ${ }^{(3)} D$. Pérez; ${ }^{(4)}$ J. \\ Rodríguez Gómez; ${ }^{(2)}$ C. Bousoño; ${ }^{(5)}$ M. Gueimonde; ${ }^{(5)}$ A. Margolles; \\ (2) J. Díaz. \\ (1)Department of Microbiology and Biochemistry of Dairy \\ Products. Dairy Research Institute of Asturias. Spanish National \\ Research Council (IPLA-CSIC). Villaviciosa. Asturias. Spain.; \\ ${ }^{(2)}$ Gastroenterology and nutrition paediatric section. Hospital \\ Universitario Central de Asturias (HUCA). Asturias. Spain; \\ ${ }^{(3)}$ Paediatric service. Hospital Universitario de Cabueñes. \\ Asturias. Spain; ${ }^{(4)}$ Department of nutrition. Food science and \\ food technology. Universidad Complutense de Madrid. Madrid. \\ Spain.; ${ }^{(5)}$ Department of Microbiology and Biochemistry of Dairy \\ Products. Instituto de Productos Lácteos de Asturias (IPLA-CSIC). \\ Group Diet, Microbiota and Health. Instituto de Investigación \\ Sanitaria del Principado de Asturias (ISPA). Oviedo. Spain.
}

Introduction: Cow`s milk protein allergy (CMPA) is the most common food allergy in infancy although a great proportion of children acquire tolerance before 3 years old, when a stable adult-like gut microbiota is being established. Associations between food allergy in infants and altered intestinal microbiota structure have been previously pointed out. However, non-IgE mediated forms are less studied and the responsible immune mechanisms remain obscure.

Objectives: The purpose of this work was to give clues on the role of microbiota and immune maturation in non-IgE CMPA. We analyzed the microbiota and associated immunological parameters in the faeces of infants with this pathology and compared the determinations with those from a control group.

Methods: Eighteen infants between 1 and 2 years old, diagnosed with non-IgE CMPA were recruited at different hospitals in

Ann Nutr Metab 2018;72 (suppl 1): 1-76 
Asturias. They were following a diet avoiding milk consumption and provided stool samples for the study. As control, a group of 10 age- matched healthy infants with normal diet were included.

Results: Differences in the composition of the gut microbial communities between CMPA and healthy children were observed. Non-allergic infants under conventional diet showed a significantly higher proportion of Bacteroides as compared to CMPA infants. Moreover, Prevotella genus was absent in this last group.

The levels of the inflammatory mediator IFN-gamma were higher in infants with CMPA meanwhile the TGF-beta, related with Treg cell response, was significantly higher in the control group.

Conclusions: Microbial colonization in non-IgE CMPA infants seems to be altered with a delayed establishment of the Bacteroidaceae family. These taxa participate in the metabolism of polysaccharides from diet resulting in the final production of SCFAs that exert anti-inflammatory effects and influence the immune system development and function.

References: Arrieta et al. 2014. The intestinal microbiome in early life: health and disease. Front Immunol 5:427

Conflict of interest: All authors declare that no competing interest exits.

\section{$309 / 27$ \\ A PRELIMINARY STUDY OF THE UPPER BOWEL MICROBIOTA IN PATIENTS WITH SIBO}

\author{
${ }^{(1)}$ N. Molinero García; (2) M. Poca; ${ }^{(2)} \mathrm{C}$. Guarner-argente; ${ }^{(2)} \mathrm{C}$. Gely; ${ }^{(2)} \mathrm{C}$. \\ Guarner; ${ }^{(1)}$ A. Margolles; ${ }^{(3)} R$. Tojo; ${ }^{(2)}$ G. Soriano; ${ }^{(1)} P$. Ruas Madiedo; \\ (1)S. Delgado. \\ (1)Department of Microbiology and Biochemistry of Dairy \\ Products. Instituto de Productos Lácteos de Asturias (IPLA-CSIC). \\ Villaviciosa. Asturias. Spain; (2)Department of Gastroenterology. \\ Hospital de la Santa Creu i Sant Pau. CIBER-EHD. Barcelona. \\ Spain; ${ }^{(3)}$ Departament of Gastroenterology. Hospital \\ Universitario de Cabueñes. Gijón. Asturias. Spain.
}

Introduction: Small intestinal bacterial overgrowth (SIBO) is a heterogeneous syndrome characterized by an increased number of bacteria in the small bowel. SIBO is diagnosed when bacterial cultures of upper intestine aspirates are $\geq 105 \mathrm{CFU} / \mathrm{ml}$. Although investigation of these aspirates is considered the gold standard, non-invasive hydrogen breath test is commonly used for SIBO diagnosis. This syndrome has been related to a decrease of small bowel motility, an increase of intestinal permeability and bacterial translocation, being associated with serious diseases such as liver cirrhosis.

Objectives: To investigate the alterations in the bacterial communities of the small bowel from SIBO patients and the potential link with microbiota from other sites (saliva and feces).

Methods: Duodenal aspirates from patients diagnosed with SIBO, control subjects and cirrhotic patients, with and without SIBO, were used to determine bacterial load (by quantitative PCR) and composition (by Illumina sequencing of $16 \mathrm{~S}$ rDNA amplicons). Saliva and feces from cirrhotic patients were also included for comparative purposes.
Results: Bacterial quantification by $\mathrm{qPCR}$ revealed counts higher than $5 \times 106$ genome equivalents $/ \mathrm{ml}$ of duodenal aspirates in SIBO patients whereas in the control subjects were below 106 . Bacterial composition was represented by members of the Lactobacillales order, diverse families of Protebacteria and genera belonging to Prevotellaceae, Veillonellaceae and Micrococcaceae families. No statistical differences were seen between the taxons presented in SIBO and non-SIBO cirrhotic patients.

Conclusions: A further investigation with a broad number of patients is needed to confirm whether: i) the usual methods employed for SIBO diagnosis are reliable, and ii) the microbiota of duodenal aspirates in cirrhosis is more closely related to that of the oral cavity than the distal gut.

References: Bures et al., 2010. Small intestinal bacterial overgrowth syndrome. World J Gastroenterol 16:2978-2990.

Conflict of interest: All authors declare that no competing interest exits.

\section{9/35 \\ GALACTO-OLIGOSACCHARIDES DERIVED FROM LACTULOSE PROTECTS AGAINST COLORECTAL CANCER DEVELOPMENT IN AN ANIMAL MODEL}

\author{
(1)J. Fernández; ${ }^{(2)}$ F. Moreno; ${ }^{(2)}$ A. Olano; ${ }^{(3)}$ A. Clemente; ${ }^{(1)} \mathrm{C}$. Villar; ${ }^{(1)} \mathrm{F}$. \\ Lombó. \\ (1)Department of Functional Biology. University of Oviedo. \\ Oviedo. Spain.; (2)Department of Bioactivity and Food Analisis. \\ Research Institute in Food Sciences CIAL (CSIC-UAM). Madrid. \\ Spain.; ${ }^{(3)}$ Department of Physiology and Biochemistry of Animal \\ Nutrition. Experimental Station of Zaidin (CSIC). Granada. Spain.
}

Introduction: Colorectal cancer has become one of the leading causes of cancer-related mortality worldwide; consequently, much attention has been focused on preventive and therapeutic strategies. In this sense, prebiotics have been reported as potential anti-inflammatory and anti-carcinogenic agents within the gastrointestinal tract. In this work, we have investigated the protective effect of novel galacto-oligosacharides derived from lactulose (GOS-Lu). These prebiotics have shown strong bifidogenic properties in vivo (Clemente et al., 2011; Hernández-Hernández et al., 2012).

Objectives: GOS-Lu has been tested here as potential antitumor preventive prebiotic, in a rat model developed with azoxymethane (AOM) as chemical inducer of colorectal cancer.

Methods: Male Fisher 344 rats were randomly assigned to two groups ( $\mathrm{n}=10$ per group, including 2 rats lacking colorectal cancer AOM induction): one cohort (negative control) with drinking water, and experimental cohort with $10 \%$ GOS-Lu (w/v) added to drinking water. Eight animals in each cohort received two doses of AOM $(10 \mathrm{mg} / \mathrm{kg})$ plus two treatments with DSS (3\% and $2 \%)$. After 17 weeks, animals were sacrificed and colon tissues analyzed for number of tumors and tumor mucosa affected area.

Results: Anatomical studies showed that caecum weight was enlarged a $35.2 \%$ on average $(\mathrm{p}<0.05)$ in experimental animals fed 9th Workshop SEPyP 
GOS-Lu. The number of colon tumors was decreased $57.5 \%$ on average $(\mathrm{p}<0.05)$ in prebiotic-fed animals. In addition, the tumor affected area in colon mucosa was reduced a $50.5 \%$ on average $(\mathrm{p}<0.05)$ in animals drinking GOS-Lu in water.

Conclusions: These findings suggest that inclusion of the prebiotic preparation GOS-Lu might contribute to the prevention of this important neoplasia.

References: Clemente A, Rubio LA, Sanz Y, Laparra JM, Sanz ML, Hernandez O, Montilla A, Olano A, Moreno FJ. Spanish patent P201130784. 2011.

Hernández-Hernández O, Marín-Manzano MC, Rubio LA, Moreno FJ, Sanz ML, Clemente A. J Nutr. 2012;142:1232-1239.

Conflict of interest: Authors declare absence of conflict of interest.

\section{$309 / 38$ \\ MATURATION OF ORAL MICROBIOTA IN RELA- TION TO ALLERGY DEVELOPMENT AND IDENTI- FICATION OF POTENTIAL PROBIOTICS}

\author{
${ }^{(1)}$ M. Dzidic; ${ }^{(2)}$ M. Collado Amores; ${ }^{(3)}$ T. Abrahamsson; ${ }^{(4)}$ A. Artacho; \\ ${ }^{(5)}$ M. Jenmalm; ${ }^{(6)}$ A. Mira. \\ (1)Institute of agrochemistry and food technology. IATA- \\ CSIC. Valencia. Spain.; ${ }^{(2)}$ Institute of Agrochemistry and Food \\ Technology (IATA) Spanish Research Council (CSIC). Valencia. \\ Spain.; ${ }^{(3)}$ Department of clinical and experimental medicine. \\ Division of pediatrics. Linköping University. Linköping. Sweden. \\ Spain.; ${ }^{(4)}$ Department of health and genomics, center for \\ advanced research in public health. csisp-fisabio. Valencia. \\ Spain.; ${ }^{(5)}$ Department of clinical and experimental medicine, \\ division of autoimmunity and immune regulation. Linköping \\ University. Linköping. Sweden.; ${ }^{(6)}$ Department of Health and \\ Genomics. Center for Advanced Research in Public Health. \\ FISABIO Foundation. Valencia. Spain.
}

Introduction: Information on how the oral microbiome develops during early childhood and how external factors influence this process is scarce. Even though the oral cavity is the first site of encounter between a majority of foreign antigens and the immune system, the influence of oral bacteria on allergy development has not yet been reported.

Objectives: We aimed to characterise oral bacteria composition in saliva samples collected at 3, 6, 12, 24 months and 7 years of age in 90 longitudinally followed children, for whom clinical, dietary and allergy data were collected.

Methods: Illumina sequencing of the $16 \mathrm{~S}$ rDNA gene.

Results: Bacterial diversity and richness increased from birth throughout childhood. Bacterial composition patterns changed through time, and "early" and "late" colonizers could be clearly detected. Infants born by C-section had initially skewed bacterial content compared to vaginally delivered infants, but this was recovered with age. Shorter breastfeeding habits and antibiotic treatment during the first 2 years of age were both associated with distinct bacterial composition at lat-

9th Workshop SEPyP er age. Children developing allergies had distinctive abundance of several bacterial species in early life together with highly divergent bacterial composition at 7 years of age, when compared to children staying healthy. Children staying healthy up to 7 years of age had several bacterial species at significantly higher levels throughout this period, including Lactobacillus gasseri and L.crispatus.

Conclusions: Data obtained are consistent with oral microbiota development during childhood being an ecological succession. A clearly altered, less diverse oral microbiota was observed in allergic children, suggesting that early changes in microbial composition seem to influence immune maturation and allergy development. The association of several lactobacilli with reduced allergy development suggests their use as potential probiotics, whose impact on the child's health and allergy risk reduction should be further studied.

References: Gomez A.2017

Conflict of interest: The authors declare that they have no competing interests.

\section{$309 / 49$ \\ PHENOLIC METABOLITES IN HUMAN FECAL SAMPLES: ASSOCIATION WITH INTESTINAL MI- CROBIOTA AND SERUM BIOMARKERS}

\author{
(1)S. González Solares; (1)I. Gutíerrez Díaz; ${ }^{(1)}$ T. Fernández Navarro; \\ ${ }^{(2)}$ N. Salazar Garzo; ${ }^{(3)}$ B. Bartolomé Suáldea; ${ }^{(3)}$ M. Moreno Arribas; \\ ${ }^{(4)} \mathrm{C}$. González de los Reyes Gavilan; ${ }^{(2)}$ M. Gueimonde.
}

(1)Department of Functional Biology. University of Oviedo. Oviedo. Spain. Group “Diet Microbiota and Health". Instituto de Investigación Sanitaria del Principado de Asturias (ISPA). Oviedo. Spain.; (2)Department of Microbiology and Biochemistry of Dairy Products. Instituto de Productos Lácteos de Asturias (IPLA-CSIC). Group Diet, Microbiota and Health. Instituto de Investigación Sanitaria del Principado de Asturias (ISPA). Oviedo. Spain.; ${ }^{(3)}$ Institute of Food Science Research (CIAL). CSIC-UAM. CEI UAM-CSIC. Madrid. Spain.; ${ }^{(4)}$ Microbiology and Biochemistry Department. Dairy Research Institute of Asturias (IPLA-CSIC). Villaviciosa-Asturias. Spain. Diet. Microbiota and Health Group. Health Research Institute of Principado de Asturias (ISPA).

Oviedo. Spain.

Introduction: Most of the health effects attributed to polyphenols may be associated with their phenolic microbial-derived metabolites, some of which could contribute to increase the antioxidant capacity of the plasma and/or to beneficially impact on gut microbial ecosystem and function (1). Therefore, the characterization of fecal microbial-derived phenolics is of interest for a better understanding of polyphenol metabolism by gut bacteria, and its consequences for human health.

Objectives: To examine, in a sample of mature adults without a declared pathology, the relationship between the excreted fecal phenolic metabolites, the composition and metabolic activity of the intestinal microbiota and selected blood parameters related to oxidative stress and inflammation. 
Methods: Blood and fecal samples from 71 healthy adults (70.83 \pm 11.12 years old) were collected. In the fecal samples, PCR of the 16S rRNA gene was used for the quantification of major bacterial groups, analysis of SCFA was performed by gas chromatography, and UPLC-ESI-MS/MS was conducted for the analysis of fecal phenolic metabolites. In blood samples, the levels of immunological markers were quantified by flow cytometry except for TGF- $\beta$ determined by ELISA.

Results: Total phenolic metabolite content was inversely associated with the fecal levels of Bacteroides group, Clostridium cluster XIVa and propionate, and directly related to Lactobacillus group and acetate. The results obtained pointed to a direct association for TGF- $\beta$, IL-17 and IL- 8 levels with the total phenolics excretion in feces, with independence of age, energy intake, physical activity or BMI. Phenylacetic and phenylpropionic acids were the major fecal phenolic compounds, accounting for 46.7 and $35.4 \%$, respectively, of the total phenolics excreted.

Conclusions: The reported association between the amounts of fecal phenolics metabolites, serum biomarkers and gut microbial composition will provide new approaches to promote the maintenance of health status.

References: Aura, AM et al. Phytochemistry Reviews 7, 407429 (2008).

Conflict of interest: No conflict of interest

\section{9/57 \\ DETECTION OF FUNGI IN HUMAN BREASTMILK ACROSS GEOGRAPHIC LOCATIONS AND EFFECT OF MODE OF DELIVERY}

\author{
${ }^{(1)}$ A. Boix-Amorós; ${ }^{(2)}$ E. du Toit; ${ }^{(3)}$ Y. Zhang; ${ }^{(4)} B$. Yang; ${ }^{(5)}$ S. Salminen; \\ ${ }^{(6)}$ E. Isolauri; ${ }^{(7)}$ A. Mira; ${ }^{(8)}$ M. Collado Amores. \\ (1)Department of Biotechnology. Institute of Agrochemistry \\ and Food Technology. Spanish National Research Council \\ (IATA-CSIC).; ${ }^{(2)}$ Division of Medical Microbiology. Department \\ of Pathology. University of Cape Town. South Africa.; \\ ${ }^{(3)}$ Department of Nutrition and Food Hygiene. School of Public \\ Health. Peking University. Beijing. China.; ${ }^{(4)}$ Food Chemistry \\ and Food Development. Department of Biochemistry. \\ University of Turku. Finland.; ${ }^{(5)}$ Functional Foods Forum. Faculty \\ of Medicine. University of Turku. Finland.; ${ }^{(6)}$ Department of \\ Pediatrics. University of Turku. Finland.; ${ }^{(7)}$ Department of \\ Health and Genomics. Center for Advanced Research in Public \\ Health. FISABIO Foundation. Valencia. Spain.; ${ }^{(8)}$ Institute of \\ Agrochemistry and Food Technology (IATA) Spanish Research \\ Council (CSIC). Valencia. Spain.
}

Introduction: Breastfeeding is essential to guide microbiome development in infants and immune system, metabolic and neural programming. Breastmilk contains a broad range of nutrients and bioactive compounds, and hosts a variety of microbes that are transmitted to the baby during breastfeeding. Recently, the presence of fungi in milk from healthy mothers has been described(1), suggesting that breastmilk could also favour the infant mycobiota development. Geographic location has shown to impact bacterial composition in breastmilk, and mode of delivery has also been suggested to influence it.

Objectives: We aimed to characterize the fungal composition in breastmilk from healthy mothers from four different countries: Spain, South Africa, Finland and China and the impact of the delivery mode.

Methods: A total of 80 volunteers participated in the study, with equal number of vaginal and $\mathrm{C}$-section deliveries per country. Fungal composition was assessed through sequencing of the ITS1 region.

Results: The two most abundant phyla in all countries were Basidiomycota and Ascomycota, with Malassezia and Davidiella being the dominant genera. Discriminant Analysis of Principal Components showed that South African samples had the most diverse mycobial profile compared to the other countries. A core of 4 genera was shared among the four geographic locations, including: Malassezia, Davidiella, Sistotrema and Penicillium. Mode of delivery also impacted on the mycobiota. In Chinese samples, Candida smithsonii was significantly more abundant in vaginal deliveries; Sistotrema sp. in C-section Spanish samples; Malasezzia restricta in Finnish vaginal samples and Ascomycota_sp in C-sections; and Malassezia restricta and Davidiella tassiana in C-sections from South African samples (LefSe analysis, $\mathrm{p}<0.05$ ).

Conclusions: The present data extend the rational of breast milk as a model of infant feeding. Future work should study the origin of these fungi and their potential contribution to infant health.

References: Boix-Amorós. A (2017).

Conflict of interest: Authors declare no conflicts of interest.

\section{9/71 \\ EXTRACELLULAR PROTEINS OF LACTOBACILLUS ACIDOPHILUS DSM 20079 DOWNREGULATES PRO-INFLAMMATORY MEDIATORS IN IBD PA- TIENTS}

\begin{abstract}
${ }^{(1)}$ C. Hidalgo-Cantabrana; ${ }^{(1)}$ M. Moro-García; ${ }^{(2)}$ A. Blanco-Míguez; ${ }^{(3)}$ F. Fernández-Riverola; ${ }^{(3)} A$. Lourenço; ${ }^{(4)}$ S. Riestra; ${ }^{(5)} R$. Alonso-Arias; ${ }^{(6)} B$. Sánchez García.
\end{abstract}

(1)Department of Microbiology and Biochemistry. Dairy Research Institute of Asturias. Spanish National Research Council (IPLA-CSIC). Immunology Department. Central University Hospital of Asturias (HUCA). Asturias. Spain.; ${ }^{(2)}$ Department of Microbiology and Biochemistry. Dairy Research Institute of Asturias. Spanish National Research Council (IPLA-CSIC). ESEIDepartment of Computer Science. University of Vigo. Spain.; ${ }^{(3)} \mathrm{ESEI}$ - Department of Computer Science. University of Vigo. Biomedical Research Centre (CINBIO). University of Vigo. Spain.; ${ }^{(4)}$ Digestive System Service. Unit of Inflammatory Bowel Disease. Central University Hospital of Asturias (HUCA). Oviedo. Asturias. Spain.; ${ }^{(5)}$ Immunology Department. Central University Hospital of Asturias (HUCA).Oviedo. Spain.; ${ }^{(6)}$ Department of Microbiology and Biochemistry. Dairy Research Institute of Asturias. Spanish National Research Council (IPLA-CSIC). Asturias. Spain. 
Introduction: Bifidobacteria and lactobacilli include both probiotic strains and representative microorganisms of the human gut microbiota, and their presence has been associated with a proper immune development and maturation. In addition, several independent works report the anti-inflammatory properties of several strains of those groups. However, we are far from understanding the molecular interplay behind/supporting these beneficial interactions.

Objectives: To study the effect of different fractions of lactobacilli and bifidobacteria in human peripheral blood mononuclear cells (PBMCs) isolated from healthy donors and patients with Inflammatory Bowel Disease (IBD) at the time of diagnosis.

Methods: The fractions were obtained from strains of Bifidobacterium longum NCIMB 8809, Lactobacillus rhamnosus GG and Lactobacillus acidophilus DSM 20079: DNA, surface-associated proteins, whole cell soluble extract and extracellular proteins. Different concentrations of the fractions were incubated with PBMCs, and key T-cell cytokines, $\mathrm{T}$ regulatory cell differentiation and RNASeq of CD4+ cells were performed.

Results: Among the extracts, extracellular proteins from $L$. acidophilus DSM 20079 increased IL-2 production by PBMCs isolated from healthy donors, and were able to increment the proportion of functional T regulatory cells. Data on the signaling mechanisms involved were obtained by RNASeq of CD4+ Th cell subpopulations. Finally, this fraction decreased drastically the production of the pro-inflammatory mediator TNF-alpha and induced increments of IL-12 in PBMCs isolated from IBD patients at the diagnosis.

Conclusions: Extracellular proteins from strain L. acidophilus DSM 20079 had a positive impact on the immunological status of both healthy donors and IBD patients. This fraction will be on the basis of new clinical studies focused in the immunomodulation of the human host targeting pro-inflammatory effectors and the $\mathrm{T}$-cell regulatory response. This fraction may represent a new therapeutic approach for chronic inflammatory illnesses.

References: Bernardo et al (2012) PLoS ONE7(5): e36262

Conflict of interest: Borja Sánchez and Claudio Hidalgo are members of the scientific committee of Microviable Therapeutics SL.

\section{Track 4: Veterinary}

\author{
Reviewers: \\ Teresa Requena Rolanía \\ Research Institute in Food Sciences, CIAL (CSIC- UAM). Madrid.
}

Juan Evaristo Suárez Fernández

Oviedo University. Asturias.

Juan Miguel Rodríguez Gómez

Veterinary School. Universidad Complutense de Madrid.

Abelardo Margolles Barro

Institute of Dairy Products of Asturias, IPLA-CSIC. Oviedo.

\section{$309 / 15$ \\ USE OF RUMEN MICROBIOTA AS PROBIOTICS FOR WEANING OPTIMIZATION IN GOAT KIDS}

\author{
A. Belanche Gracia; I. Nejjam; J. Palma; R. Serrano; E. Jiménez; A. \\ Martín García; D. Yáñez Ruiz.
}

Estación Experimental del Zaidín. CSIC. Armilla. Granada. Spain.

Introduction: Young ruminants present at birth an undeveloped rumen which requires an anatomical, physiological and microbial development to fully stablish its function as a fermentation chamber. This process can be jeopardized in animals with artificial milk feeding due to the absence of a direct contact with adult animals which represent the main source of rumen microbiota. Thus, further strategies are needed to accelerate the rumen microbial development on such situations and to optimize the weaning process.

Objectives: This study investigates the use of rumen microbiota as probiotics as a strategy to accelerate the rumen development.

Methods: A total of 64 new born goat kids were randomly distributed in 4 treatments $(n=16)$ : animals were orally inoculated with fresh rumen fluid from either goats fed a concentrate (CRF) or forage diet (FRF), autoclaved rumen fluid as prebiotic (PRE), or absence of inoculation as control (CTL). Inoculation was daily performed at a rate of $0.5 \mathrm{~mL} / \mathrm{kg}$ body weight from birth until 12 weeks of age. Animals had free access to milk replacer, concentrate feed and hay. Rumen samples were taken at 5, 7 and 9 weeks of age being equivalent to early weaning, real weaning and post-weaning, respectively.

Results: Inoculation with fresh rumen fluid promoted an acceleration in the rumen microbial development in terms of concentration of rumen protozoa, methanogens and anaerobic fungi $(\mathrm{P}<0.05)$. As a result, an increase in the rumen volatile fatty acid concentration $(+51 \%)$ was noted at 5 weeks. Although these differences disappeared during successive sampling times, animals inoculated with fresh rumen fluid showed increased forage intake and greater growth rate during the weaning process.

Conclusions: Inoculation of goat kids with fresh rumen fluid from adult animals can be considered as a strategy to accelerate the rumen development and to optimize weaning process in animals reared under artificial lactation.

Conflict of interest: Authors declare no conflict of interest.

Ann Nutr Metab 2018;72 (suppl 1): 1-76 



\section{Poster Abstract Presentations}

\section{Nutritions Metabolism}

\section{Track 1: Immunonutrition}

\section{Reviewers:}

Ascensión Marcos Sánchez

Institute of Science and Technology of Food and NutritionICTANCSIC. Madrid.

Mónica de la Fuente del Rey

Biological Sciences Department. Universidad Complutense de Madrid.

José Manuel Martín Villa

School of Medicine. Universidad Complutense de Madrid.

\section{$309 / 10$}

\section{EFFICACY OF A BIFIDOBACTERIUM INFANTIS CECT7210 BASED SYNBIOTIC AGAINST ETECK88 IN A PIG MODEL}

\author{
${ }^{(1)}$ A. Rodríguez; ${ }^{(1)}$ L. Castillejos Velázquez; ${ }^{(1)}$ P. López Colom; ${ }^{(2)} G$. \\ Cifuentes Orjuela; ${ }^{(2)}$ J. Moreno Muñoz; ${ }^{(1)}$ S. Martín Orúe.
}

(1) Animal Nutrition and Welfare Service. Animal and Food Science Department. Autonomous University of Barcelona. Barcelona. Spain.; (2) Departament of Basic Research. ORDESA Laboratories. Barcelona. Spain.

Introduction: One of the most important and well documented effects of probiotics is their role in the defense against pathogenic microorganisms in the gut. Enterotoxigenic Escherichia coli $s p p$. (ETEC) are among the most common bacterial causes of diarrhea associated morbidity and mortality, especially in children up to 5 years of age (1).

Objetive: This study was aimed to evaluate the efficacy of a synbiotic combination against enterotoxigenic Escherichia coli (ETEC) K88. This compound consisted of Bifidobacterium longum subsp. infantis CECT 7210 (Laboratorios Ordesa S.L.) with inulin and oligofructose.

Methods: 96 piglets of 21 days were distributed into 32 pens in a $2 \times 2$ design: with or without synbiotic, inoculated or not with the pathogen. Animals received the probiotic $\left(10^{\wedge} 9 \mathrm{cfu} / \mathrm{animal}{ }^{\star}-\right.$ day) and the prebiotic (5\%) in the feed. After one week of adaptation, the animals were orally inoculated with ETEC K88 (K88ab, K88ac, LT, STb, EAST1) at a single dose of 1.2x1010cfu. Food intake, weight gains, fecal consistency and rectal temperature were recorded. On days 3 and 7 post-inoculation (PI), one animal per pen was euthanized and blood, feces and tissue samples were collected. Enterobacteria and coliform counts in ileum mucosa scrap- ings and faeces, and serum levels of TNF- $\alpha$ and PigMAP were analyzed.

Results: There was a trend to reduce weight gains in the PI period with the synbiotic ( 227 vs. $267 \mathrm{~g} / \mathrm{d}, \mathrm{P}=0.13$ ). Clinical signs, fecal counts of enterobacteria and coliforms, and TNF- $\alpha$ and PigMAP responded to inoculation but not the treatment. Regarding ileum scrapings, a decrease of enterobacteria and coliforms was observed with the synbiotic in the non-inoculated animals at day 7 PI (enterobacteria: 7.98 vs. 9.25; coliforms: 7.86 vs. $8.21 \mathrm{cfu} / \mathrm{g}$ ), but the contrary in the challenged animals (enterobacteria: 8.53 vs. 7.08; coliforms 8.55 vs. $6.70 \mathrm{cfu} / \mathrm{g}$ ) (Interaction $\mathrm{P}=0.057, \mathrm{P}=0.104$ respectively).

Conclusions: The results show that this synbiotic was not able to compensate for the negative effects of an oral ETEC K88 challenge, but neither to exacerbate them, suggesting being safe in dysbiosis. More studies are guaranteed to evaluate the response observed in the ileum adhered enterobacteria.

References: Barba-Vidal, E. (2016) y Guerra-Ordaz, A.A. (2013), Tesis Doctorales (UAB)

Conflict of interest: Authors declare no conflict of interest.

\section{$309 / 18$}

\section{ANTIHYPERTENSIVE EFFECTS OF SHORT-CHAIN FATTY ACIDS AND PROBIOTICS CONSUMPTION IN SPONTANEOUSLY HYPERTENSIVE RATS}

\author{
I. Robles Vera; N. De La Visitación Pastor; M. Toral Jiménez; M. \\ Romero-Pérez; M. Sánchez Santos; M. Duarte Pérez; R. Jiménez \\ Moleón; J. Duarte Pérez. \\ Department of Pharmacology. School of Pharmacy. University of \\ Granada. Granada. Spain.
}

Introduction: Hypertension is associated with gut microbiome dysbiosis, characterized by low short-chain fatty acids (SCFAs), butyrate- or acetate-producing bacteria ${ }^{1}$. Therefore, the modulation of gut microbiota by the administration of SCFAs-producing bacteria may lead to the development of novel antihypertensive therapies2.

Objectives: The aim of this study was to evaluate the cardiovascular effects of probiotics Lactobacillus fermentum CECT5716 (LC40) or Bifidobacterium breve CECT7263 (BFM), and acetate and butyrate in spontaneously hypertensive rats (SHR).

Methods: Ten five-weeks old Wistar Kyoto rats (WKY) and fifty SHR were randomly assigned to six groups $(n=10)$ : a control WKY group and a control SHR group, a treated SHR-LC40 group a treated SHR-BMF group, a treated SHR-butyrate group, and a 
treated SHR-acetate group. The probiotics were orally gavaged at the final concentration of 109 colony-forming units day-1. SCFAs supplementation was given as $21,45 \mathrm{mg} \mathrm{kg}-1$ day-1acetate or 0,5 $\mathrm{mg}$ kg-1 day-1 butyrate in drinking water. The treatments were given for 13 weeks.

Results: Long-term administration of probiotics and butyrate prevented the rise in systolic blood pressure (SBP) and the cardiac hypertrophy. Both groups of probiotics and butyrate treatment improved the impaired aortic relaxation by acetylcholine in SHR. They also restored the increased oxidative stress by reducing NADPH oxidase activity. Acetate did not show protective effects in SHR.

Conclusions: This study demonstrates a cardiovascular protective effect of probiotics in SHR by increasing nitric oxide bioavailability. The butyric acid, one of the main metabolites of the gut microbiota, might be involved in the beneficial effects of probiotic in genetic hypertension.

Reference: 1. Qi et al. Gut Microbiota: Potential for a Unifying Hypothesis for Prevention and Treatment of Hypertension. Circ Res. 2017;120:1724-1726.

2. Gómez-Guzmán et al. Antihypertensive effects of probiotics Lactobacillus strains in spontaneously hypertensive rats. Mol Nutr Food Res. 2015; 59:2326-36.

Conflict of interest: Authors declare no conflict of interest.
Results: LC40 treatment reduced lupus disease activity, blood pressure, cardiac and renal hypertrophy, splenomegaly, albuminuria, and renal injury in lupus mice. LC40 reduced the elevated $\mathrm{T}, \mathrm{B}$, Treg and Th1 cells in spleen and in mesenteric lymph nodes from lupus mice. LC40 lowered the higher plasma concentration of proinflammatory cytokines observed in lupus mice. Aortae from lupus mice showed reduced endothelium-dependent vasodilator responses to acetylcholine. Endothelial dysfunction induced by SLE was related to both increased NADPH oxidase driven-superoxide production and Rho-kinase mediated eNOS inhibition, which were normalized by LC40. In addition, LC40 treatment reduced hypertension, endothelial dysfunction, and organ damage in severe lupus mice, which was associated with reduced plasma anti-double-stranded DNA autoantibodies and anti-inflammatory as well as antioxidant effects in target tissues. Probiotic administration to lupus mice reduced the metabolic endotoxemia, which was related to a significant improvement of the gut barrier disruption.

Conclusions: Our findings identify this gut microbiota manipulation as a promising target for an alternative approach in the treatment of SLE and its associated vascular damage.

Reference: 1 . Sánchez et al. Interaction of Intestinal Microorganisms with the Human Host in the Framework of Autoimmune Diseases. Front Immunol. 2015; 6:594.

Conflict of interest: Authors declare no conflict of interest.

\section{$309 / 19$ \\ CHRONIC LACTOBACILLUS FERMENTUM CT5716 IMPROVES ENDOTHELIAL DYSFUNCTION AND PROTECTS KIDNEY IN A MOUSE MODEL OF SYS- TEMIC LUPUS ERYTHEMATOSUS}

\author{
${ }^{(1)} M$. Toral Jiménez; ${ }^{(1)}$ I. Robles Vera; ${ }^{(1)} M$. Romero-Pérez; ${ }^{(1)} M$. \\ Sánchez; ${ }^{(1)}$ R. Jiménez Moleón; ${ }^{(1)} F$. O'valle; ${ }^{(1)}$ A. Rodríguez-Nogales; \\ ${ }^{(1)} N$. De La Visitación Pastor; (2) J. Gálvez; (1)J. Duarte Pérez. \\ (1)Department of Pharmacology. School of Pharmacy. University \\ of Granada. Granada. Spain.; ${ }^{(2)}$ Department of Pharmacology. \\ CIBER-EHD. ibs. Granada. CIBM. University of Granada. Granada. \\ Spain.
}

Introduction: Systemic lupus erythematosus (SLE) is a multisystemic chronic autoimmune inflammatory disorder that is associated with intestine dysbiosis1. Lactobacillus fermentum CECT5716 (LC40) is a probiotic bacterium that modulates the human immune system.

Objectives: The aim of this study was to examine whether LC40 ameliorates disease activity and cardiovascular complications in a lupus mouse model.

Methods: Eighteen-week-old NZBWF1 (lupus) and NZW/ LacJ (control) mice were treated with LC40 (5x108 CFU day-1) for 15 weeks. Blood pressure, plasmatic double-stranded DNA autoantibodies, lipopolysaccharides (LPS) and cytokines, nephritis, spleen and mesenteric lymph nodes lymphocytic populations, endothelial function, and vascular oxidative stress were compared in treated and untreated mice.

\section{$309 / 41$ \\ SERUM-FREE FATTY ACIDS AS POTENTIAL OBE- SITY BIOMARKERS ASSOCIATED WITH THE IN- TESTINAL MICROBIOTA}

\author{
(1)T. Fernández Navarro; ${ }^{(2)}$ J. Rodríguez Carrio; ${ }^{(2)}$ A. Suárez Díaz; ${ }^{(1)}$ I. \\ Gutierrez Díaz; ${ }^{(3)} \mathrm{C}$. González de los Reyes Gavilan; ${ }^{(4)} \mathrm{M}$. Gueimonde; \\ ${ }^{(4)}$ N. Salazar Garzo; ${ }^{(1)}$ S. González Solares. \\ (1)Department of Functional Biology. University of Oviedo. \\ Oviedo. Spain. Group “Diet Microbiota and Health”. Health \\ Research Institute of Principality of (ISPA). Oviedo. Spain.; \\ ${ }^{(2)}$ Departament of Functional Biology. University of Oviedo. \\ Group Autoimmunity and Inflammation. Health Research \\ Institute of Principality of (ISPA). Oviedo. Spain.; ${ }^{(3)}$ Microbiology \\ and Biochemistry Department. Dairy Research Institute of \\ Asturias (IPLA-CSIC). Villaviciosa-Asturias. Spain. Diet. Microbiota \\ and Health Group. Health Research Institute of Principality of \\ Asturias (ISPA). Oviedo. Spain.; ${ }^{(4)}$ Department of Microbiology \\ and Biochemistry of Dairy Products (IPLA-CSIC). Group Diet, \\ Microbiota and Health. Health Research Institute of Principality \\ of (ISPA). (ISPA). Oviedo. Spain.
}

Introduction: The gradual increase in energy intake and the growing adherence to Western-type diet observed during the last decades, have been linked to the increasing prevalence of obesity. Serum free fatty acids (FFA), which are closely related to fat intake, are generally elevated in obesity. Gut microbiota is already known to be involved in the host energy metabolism through the regulation of body fat storage1, and also to be linked to FFA2 
Objectives: To explore the association between serum FFA levels, gut microbiota and obesity.

Methods: 66 subjects (age $52.7 \pm 11.2 y$ ) were classified according to BMI. Levels of the major phylogenetic types of the intestinal microbiota were determined by qPCR. Total serum FFA were analysed by a colorimetric enzymatic assay and individual FFA were assessed by a methyl-tert-butylether-based extraction protocol (MTBE).

Results: Mean serum levels of eicosapentaenoic acid ( $\omega-3$ series) were lower in the obese group while linoleic and $\gamma$-linolenic acid ( $\omega-6$ series) were higher in comparison to normal-weight subjects. The intestinal microbial groups Faecalibacterium prausnitzii, Bacteroides and Lactobacillus were identified as linoleic acid predictors in a multiple regression model adjusted by gender. Since Bacteroides were found to be inversely related to both, linoleic acid and obesity may be envisaged.

Conclusions: These preliminary results reveal the duality of serum omega- 3 and omega- 6 fatty acids as potential biomarkers of obesity supporting the pivotal role of Bacteroides in this complex interrelationship.

References: 1.Bäckhed $F$, et al. The gut microbiota as an environmental factor that regulates fat storage. Proc Natl Acad Sci U S A. 2004;101(44):15718-15723. doi:10.1073/pnas.0407076101.

2. Rodríguez-Carrio J, et al. Free fatty acids profiles are related to gut microbiota signatures and short-chain fatty acids. Front Immunol. 2017;8(JUL). doi:10.3389/fimmu.2017.00823.

Conflict of interest: Authors declare no conflict of interest.

\section{$309 / 53$ \\ EARLY LIFE SUPPLEMENTATION WITH TWO STRAINS OF LACTOBACILLUS PARACASEI INFLU- ENCES THE RAT INTESTINAL IMMUNE SYSTEM}

\footnotetext{
${ }^{(1)}$ M. Abril; (1)L. Marín Morote; ${ }^{(2)}$ C. De Almagro; ${ }^{(2)}$ G. Cifuentes Orjuela; ${ }^{(2)}$ J. Moreno Muñoz; ${ }^{(2)}$ M. Rodríguez Palmero; ${ }^{(1)}$ M. Castell Escuer; ${ }^{(1)} \mathrm{F}$. Pérez Cano; ${ }^{(1)}$ À. Franch Masferrer.

(1)Departament of Biochemistry and Physiology. Faculty of Pharmacy and Food Sciences. University of Barcelona. Institute for Research on Nutrition and Food Safety (INSA.UB). University of Barcelona. Barcelona. Spain.; ${ }^{(2)}$ Departament of Basic Research. ORDESA Laboratories. Barcelona. Spain.
}

Introduction: Early infancy is a critical period for immune system development, and thus is a window of time that could be interesting for the use of probiotics to promote its maturation ${ }^{1}$.

Objectives: The aim of the present work was to ascertain whether the early life supplementation with two strains of Lactobacillus paracasei could influence rat intestinal immune system maturation.

Methods: Wistar rats were supplemented with two specific strains of L. paracasei (ORD0681 and ORD0712) during the first 4 weeks of life. At the end of the study, lymphocytes from mesenteric lymph nodes (MLN) were isolated in order to determine their Th1/Th2 cytokine secretion after stimulation with anti-CD3/

9th Workshop SEPyP
CD28 antibodies. Moreover, a fragment of small intestine was obtained to evaluate the intestinal expression of several genes related to the interaction bacteria-host (TLR-2, -4, -5, -7 and -9), the innate immune defence (mucin-2 and -3 ) and the epithelial barrier integrity (ZO-1, occludin, claudin-2 and -4) by Real Time-PCR.

Results: The early life supplementation with both $L$. paracasei strains significantly decreased IL-4 and IFN-y production in stimulated MLN lymphocytes, in comparison with the reference group. However, only the ORD0712 strain was able to increase IL-5 secretion. Regarding the intestinal gene expression, the $L$. paracasei ORD0712 strain overexpressed claudin-2 and tended to increase TLR-4 gene expression, whereas the ORD0681 strain only induced a decrease of TLR-2. The mucin gene expression was not modified by these probiotics.

Conclusions: These results evidence that rat supplementation with two strains of Lactobacillus paracasei, throughout the suckling and early post-weaning periods, has a strain-dependent immunomodulatory effect that could contribute to the maturation of intestinal immune system.

References: 1.Martin R, et al. Early life: Gut microbiota and immune development in infancy. Benef Microbes 2010.

Conflict of interest: The authors -CDA, GCO, JAMM, MRP- declare that they are employees of the company Laboratorios Ordesa.

\section{9/64 \\ SCGOS/LCFOS SUPPLEMENTATION TO SUCK- LING RATS PROTECTS FROM ROTAVIRUS INFEC- TION BY PROMOTING INTESTINAL MATURATION AND INFLUENCING MICROBIAL ECOSYSTEM}

\begin{abstract}
(1)I. Azagra Boronat; ${ }^{(1)}$ M. Massot-Cladera; ${ }^{(1)}$ P. Ruiz; ${ }^{(1)}$ A. Franch; $;{ }^{(1)}$ M. Castell; ${ }^{(1)}$ M. Rodríguez Lagunas; ${ }^{(2)}$ K. Van Limpt; ${ }^{(2)}$ K. Knipping; ${ }^{(2)} \mathrm{J}$. Garssen; ${ }^{(3)}$ F. Pérez Cano.

(1)Physiology section. Faculty of Pharmacy and Food Sciences. University of Barcelona. Barcelona. Spain.; ${ }^{(2)}$ Danone Nutricia Research. The Netherlands.; ${ }^{(3)}$ Departament of Biochemistry and Physiology. Faculty of Pharmacy and Food Sciences. University of Barcelona. Institute for Research on Nutrition and Food Safety (INSA.UB). University of Barcelona. Barcelona. Spain.
\end{abstract}

Introduction: In previous studies we have demonstrated that a 9:1 prebiotic mixture of short-chain galactooligosaccharides (scGOS) and long-chain fructooligosaccharides (lcFOS) could be a good strategy to prevent clinical symptoms associated to rotavirus (RV) infection ${ }^{1}$. However, the precise mechanisms of action of these early life prebiotics deserve to be studied.

Objectives: Thus, the aim of this work was to investigate the mechanisms involved in the preventive effect of scGOS/lcFOS supplementation in the RV-induced diarrhoea in rats.

Methods: Lewis rats were supplemented either with scGOS/ lcFOS or vehicle, and intragastrically RV-infected. Faecal samples were daily collected for its clinical evaluation. Intestines were obtained, weighed and measured. Moreover, jejunum sections were paraffin-embedded and eosin/hematoxylin stained to study the 
histological architecture. Small intestine expression of genes related to maturation by $\mathrm{qPCR}$, caecal microbiota composition by metagenomic analysis and SCFA production by GC-MS was also studied.

Results: The amelioration of the viral gastroenteritis due to supplementation with scGOS/lcFOS could be due to a trophic effect on the intestine which is based in an increase of weight and length of small and large intestines, larger villi and higher gene expression levels of intestinal maturation biomarkers. Finally, several changes in the SCFA production were detected, which might be connected to some of the changes of the microbiota composition; scGOS/lcFOS supplementation was able to avoid some aspects of the dysbiosis induced by the RV infection.

Conclusions: These results evidence that the amelioration of the RV-diarrhoeic process by scGOS/lcFOS, may be due to a trophic effect and accelerated maturation of the intestine, and the changes induced on the microbiota composition and activity (or metabolites).

References: 1 . Rigo-Adrover et al (2017) A combination of scGOS/lcFOS with Bifidobacterium breve M-16V protects suckling rats from rotavirus gastroenteritis. Eur J Nutr. 56(4):1657.

Conflict of interest: The authors -VL-K, G-J, K-K- declare that they are employees of the company Nutricia Research.

\title{
$309 / 78$
}

\section{CHRONIC NITRIC OXIDE BLOCKADE SUP- PRESSED THE ANTIHYPERTENSIVE EFFECT OF THE PROBIOTIC LACTOBACILLUS FERMENTUM CECT5716}

\author{
J. Duarte Pérez; N. De La Visitación Pastor; I. Robles-Vera; M. \\ Romero-Pérez; M. Sánchez Santos; M. Toral Jiménez; R. Jiménez \\ Moleón.
}

Department of Pharmacology. School of Pharmacy. University of Granada. Granada. Spain.

Introduction: Lactobacillus fermentum CECT5716 (LC40) is known to reduce blood pressure in spontaneously hypertensive rats $^{1}$.

Objectives: We tested whether the probiotic LC40 ameliorates hypertension in rats with chronic nitric oxide synthase (NOS) inhibition.

Methods: Rats were randomly divided into four different treatment groups for 4 weeks: a) vehicle (control, $1 \mathrm{ml}$ of tap water once daily), b) vehicle plus L-NAME (50 mg $100 \mathrm{ml}$-1in drinking water), c) LC40 (109 colony-forming units/day by gavage), and d) LC40 plus L-NAME. The evolution in systolic blood pressure, and morphological variables, proteinuria, lymphocytes populations in mesenteric nodes and vascular NADPH oxidase activity and vascular reactivity at the end of the experiment were analysed.

Results: LC40 did not inhibit the development of L-NAME-induced hypertension, the increase in the left ventricular hypertrophy, and proteinuria. However, this probiotic partially prevents the impaired endothelium- and NO-mediated relaxation to acetylcholine in aorta, being without effects in small mesenteric arteries.

This improvement was suppressed in presence of apocynin in the bath and was accompanied by reduced NADPH oxidase activity in both arterial beds from LC40 plus L-NAME group as compared with L-NAME group. In mesenteric lymph nodes L-NAME increases Th17 cells and reduces Treg cells. These changes were prevented by LC40 treatment. In most cases these effects were not observed in normotensive animals.

Conclusions: This study confirms the critical role of NO in the antihypertensive and end-organ protective effects of the probiotic LC40 in animal models of hypertension.

Reference: 1. Gómez-Guzmán et al. Antihypertensive effects of probiotics Lactobacillus strains in spontaneously hypertensive rats. Mol Nutr Food Res. 2015;59:2326-36.

Conflict of interest: Authors declare no conflict of interest. 


\section{Track 2: Clinical Use}

\author{
Reviewers: \\ Francisco Guarner Aguilar \\ Departament of Gastroenterology. Hospital Vall d'Hebron. \\ Barcelona. \\ Guillermo Álvarez Calatayud \\ Department of Pediatrics. Gregorio Marañón Hospital. Madrid.
}

\section{Fernando Azpiroz Vidaur}

Departament of Gastroenterology. Hospital Vall d'Hebron. Barcelona.

\section{Rosaura Leis Trabazo}

Paediatrics Professor. University of Santiago de Compostela. Gastroenterology, Hepatology and Paediatrics Nutrition Unit Coordinator at the University Hospital of Santiago.

\section{$309 / 9$ \\ DIGESTIVE DISORDERS IN THE ELDERLY AND PROBIOTICS IN THEIR PREVENTION}

\author{
${ }^{(1)}$ F. Hernández-Martinez; ${ }^{(2)}$ C. Jiménez-Rodríguez; ${ }^{(3)}$ J. Jiménez-Díaz; \\ ${ }^{(3)} B$. Rodríguez-de-Vera. \\ (1)Occupational Risk Prevention Service. Cabildo de Lanzarote. \\ Islas Canarias. Spain.; (2)Pharmaceutical. Official College of \\ Pharmaceuticals of Las Palmas de Gran Canaria. Islas Canarias. \\ Spain.; ${ }^{(3)}$ Nursing Department University of Las Palmas de Gran \\ Canaria. Islas Canarias. Spain.
}

Introduction: In the elderly the digestive affections are more frequent due to different causes. The physiological changes inherent in age, the adverse effects of certain medications and the sedentary lifestyle are the main causes of digestive and motor dysfunction. It has been shown that the intake of fermented milks with the addition of bifidobacterium, twice a day, accelerates colonic transit and regularizes the defective habit.

Objectives: To determine if the users of a Center of the Third Age of the Canary Islands who report having problems of abdominal distension and constipation, improve this symptomatology with healthy habits of life and the taking of yogurt with Bifidobacterium.

Methods: Quantitative, descriptive, observational study using an ad hoc questionnaire on a random sample of 40 people over 65 years of age after taking dairy products containing yogurt with probiotics for 15 days. Data collection period: September 2017. Data analysis with the statistical package SPSS 21.0.

Results: mean age of $71 \pm 24$ years, the age group between 70 and 80 years is the most numerous in the study and men are the most frequent sex (60\%). $85 \%$ of patient referred improvement of constipation after taking yogurts with probiotics and reduced intake of milk and refined sugars. There is a significant difference between the consumption of probiotics and chewing food during longer periods of time and the reduction of abdominal distension.

Conclusions: The combination of probiotic consumption and the acquisition of certain eating habits improve constipation in the elderly.

9th Workshop SEPyP

\section{References:}

1. Bixquert M. Manejo dietético del estreñimiento crónico funcional. Especial referencia al beneficio de las bifidobacterias. Alim Nutri Salud, 2008; 15: 31-38.

2. Zarzuelo A, Gálvez J. Fibra dietética. En: Gil A, ed. Tratado de Nutrición. Madrid: Acción Médica 2005; 337-368.

Conflict of interest: Authors declare no conflict of interest.

\section{$309 / 11$ \\ SYNBIOTIC, CONTAINING BACILLUS COAGU- LANS LMG-S-24828 AND PREBIOTICS IN THE PREVENTION OF GASTROINTESTINAL ADVERSE EFFECTS}

\author{
${ }^{(1)}$ B. Medrano Engay; ${ }^{(2)}$ C. Gómez Notario; ${ }^{(3)} J$. Alcedo; ${ }^{(4)}$ P. Giraldo \\ Castellano. \\ (1) Unit for Traslational Research.University Hospital Miguel \\ Servet. Zaragoza. Spain.; ${ }^{(2)}$ Unit for Traslational Research. \\ University Hospital Miguel Servet. Fundación Española para \\ el Estudio y Terapéutica de la Enfermedad de Gaucher y otras \\ Lisosomales. Zaragoza. Spain.; ${ }^{(3)}$ Digestive Unit . University \\ Hospital Miguel Servet. Zaragoza. Spain.; ${ }^{\left({ }^{4}\right)}$ Instituto de \\ Investigación Sanitaria Aragón. Fundación Española para el \\ Estudio y Terapéutica de la Enfermedad de Gaucher y otras \\ Lisosomales. Centro de Investigación Biomédica en Red de \\ Enfermedades Raras. Zaragoza.
}

Introduction: Some tyrosine kinase inhibitors used in the treatment of chronic myeloid leukemia, and Miglustat, an iminosugar used in lysosomal diseases, both can cause gastrointestinal disorders such as diarrhea, bloating and abdominal pain. These adverse effects decrease health-related quality of life and lead to early withdrawal of treatment. Some probiotics have shown improvement in symptoms mentioned in patients with functional digestive disorders (1).

Objectives: To evaluate the effect of controlled administration of a symbiotic, containing Bacillus coagulans LMG-S-24828 and prebiotics for one month on the quality of life related to gastrointestinal health in patients treated with ITKs and miglustat. Secondarily, to establish whether the symbiotic provides advantages in adherence to the treatments mentioned.

Methods: Randomized cross-over trial in which 15 patients on ITKs or Miglustat were blinded to placebo or symbiotic in a daily dose, with a two-month "wash" phase between administration of each from them. The patient was asked to complete the validated Spanish version of the Gastrointestinal Quality of Life Index (GIQLI) questionnaire (2) before the first dose of each product and one month after its start. The frequency of treatment withdrawal with ITKs and Miglustat in each group was also evaluated. The analysis of the results will be performed using the Student $t$ test, considering statistical significance of the differences with $p$ value $<0.05$

Results: The recruited patients are in the wash phase and the results obtained after the surveys will be provided at the meeting

Ann Nutr Metab 2018;72 (suppl 1): 1-76 
Conclusions: Will be provided at the meeting

References: (1) Simrén M, et al., Intestinal microbiota in functional bowel disorders: a Rome foundation report. Gut, 2013. 62(1): p. 159-76

(2) Eypasch E, et al., The Gastrointestinal Quality of Life Index. A clinical index for measuring patient status in gastroenterologic surgery. Chirurg, 1993. 64(4): p. 264-74.

Conflict of interest: Authors declare no conflict of interest.

\section{$309 / 25$ \\ BIDIRECTIONALITY OF THE BRAIN-GUT AXIS}

\section{J. Sánchez Núñez.}

Healthy help corporation. Pontevedra. Spain.

Introduction: Every time more studies aim to the fact that the microbiota can play an important role in the brain-gut communication, it seems factual the link between the gut microbiota, the brain and the emotional activity. In several studies it is already established the link between the gut microbiota and emotional disorders, however in these same studies is neglected the fact that this axis could be bidirectional.

Objectives: To reveal connections between the microbiota and its relation with several emotional states, also, to focus in a possible bidirectional connection (gut-brain, brain-gut) in disorders of emotional type..

Methods: Meta- analysis of published studies.

Results: The results of the several studies have worked for us in order to synthesize the results.

Conclusions: The results seem to evidence a manifest of bidirectionality (two-way connection), if well they also reveal the need to keep investigating about this particular.

\section{References:}

1. Tillisch K, Mayer E, Gupta A, et al. T, Zeevi D, Zmora N, et al. Brain structure and response to emotional stimuli as related to gut microbial profiles in healthy women. Psychosomatic Medicine. 2017 DOI: 10.1097/PSY.0000000000000493

2. Furness JB, Poole DP. Nonruminant nutrition symposium: Involvement of gut neural and endocrine systems in pathological disorders of the digestive tract. Journal of Animal Science. 2015; 90(4): 1203-1212. doi:10.2527/jas.2011-4825

3. Zhou L, Foster JA. Psychobiotics and the gut-brain axis: in the pursuit of happiness. Neuropsychiatric Disease and Treatment. 2015; 11: 715-723. doi https://dx.doi.org/10.2147/NDT.S61997

4. Dinan TG, Cryan JF. The impact of gut microbiota on brain and behaviour: implications for psychiatry. Current Opinion in Clinical Nutrition \& Metabolic Care. 2015; 18(6): 552-558. doi: 10.1097/MCO.0000000000000221

5. Mayer EA, Padua D, Tillisch K. Altered brain-gut axis in autism: comorbidity or causative mechanisms? Bioessays. 2014; 36(10):933-9. doi: 10.1002/bies.201400075.

... And other studies

Conflict of interest: There is no conflict of interest

\section{$309 / 51$ \\ EFFECTIVENESS OF PROBISAN ${ }^{\circledR}$ IN THE PREVEN- TION OF NON-OBESE DIABETIC MOUSE TYPE 1 DIABETES MELLITUS}

\section{Cabello Olmo.}

Biochemistry Area. Health Science Department. Public University of Navarra. Pamplona. Spain.

Introduction: Diabetes is a pathology with a high prevalence ${ }^{1}$. Current treatments for diabetes only alleviate side effects. The development of safe and effective treatments is a primary objective for the treatment of diabetes. Probiotics, like Probisan ${ }^{\star}$, administration offers a novel approach $_{2}$ -

Objectives: To determine whether Probisan ${ }^{\circledR}$ administration is effective in the treatment of type 1 diabetes that develops in the Non-Obese Diabetic (NOD) mouse.

Methods: Female NOD mice $(n=164)$ were organized in four experimental groups ( $\mathrm{n}=41$ mice per group):

Group1: animals fed ad libitum with standard-rodent-chow (SRC)

Group2: fed ad libitum with SRC SUPPLEMENTED with Probisan $^{\infty}$

Group3: fed ad libitum with SRC SUPPLEMENTED with Probisan $^{\circledast}$ during only the first 4 weeks of the study

Group4: fed ad libitum with SRC SUPPLEMENTED with Probisan $^{\oplus}$ only at the debut (hyperglycemia $>400 \mathrm{mg} / \mathrm{dl}$ ).

Blood glucose and body weight were analyzed twice a week and an intraperitoneal glucose tolerance test $(1.5 \mathrm{~g} / \mathrm{kg}$ body weight) was performed each month. The study lasted 40 weeks.

Results: Mice supplemented with Probisan ${ }^{\circledR}$ showed a significant reduction $(p=0.01)$ in glycaemia following an intraperitoneal glucose tolerance test. Diabetic mice in control group treated with

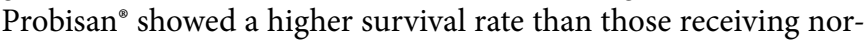
mal diet $(\mathrm{p}<0.01)$. In addition, mice treated with Probisan ${ }^{\oplus}$ showed a significant delay in the occurrence of episodes of hyperglycemia, although this did not translate into significant differences on the survival rate.

Conclusions: Supplementation with Probisan $^{\circledR}$ significantly decreased glycaemia and increased survival in diabetic mice. The administration delayed the onset of hyperglycemia although it did not prevent the development of diabetes.

\section{References:}

(1)Centers for Disease Control and Prevention. National Diabetes Statistics Report, 2017: Estimates of Diabetes and Its Burden in the United States [Internet]. Atlanta:Centers for Disease Control and Prevention; 2017.

(2)Gomes AC, Bueno AA, de Souza RGM, Mota JF. Gut microbiota, probiotics and diabetes. Nutr.J [Internet].2014;13:60

Conflict of interest: No conflicts of interest, financial or otherwise, are declared by the authors. 


\section{$309 / 61$}

\section{BACTERIAL DERIVATIVES OF LACTOCOCCUS LACTIS AND ECTOIN FOR ATOPIC DERMATITIS: DERMAL COMPATIBILITY AND COSMETIC AC- CEPTABILITY}

C. Crespo Mora.

Primary Care Pediatrics. Primary Care Center Montclar. Sant Boi de Llobregat. Barcelona. Spain.

Introduction: Atopic dermatitis (AD) is a chronic skin disease characterized by disturbance of the skin barrier function. Recent studies have shown that application of non-viable bacterial derivatives can strengthen the barrier function and reduce signs of inflammation.

Objectives: To assess skin compatibility and cosmetic acceptability of a new emollient for AD based on bacterial derivatives (lysate of Lactococcus lactis) and ectoin.

Methods: Four studies were conducted: an in vitro evaluation of irritation potential using the Hen's Egg Test on the chorioallantoic membrane (HET CAM); an allergenic potential test to confirm the absence of dermal sensitization in healthy volunteers (HRIPT); a skin compatibility study in volunteers with atopic tendency skin (Open test) and a cosmetic acceptability test in healthy pediatric volunteers. Responses in all studies were visually evaluated by a dermatologist or pediatrician, scoring potential dermatological reactions and recording any feeling of discomfort at the time of examination.

Results: The HET- CAM test showed irritation potential similar to other products in the same category. No volunteer or child participating in the studies showed any skin reactions or feelings of discomfort. HRIPT discarded allergenic potential and verified that no components induce sensitization reactions. Open test confirmed good skin compatibility in atopic skin. Finally, the test on children showed excellent tolerability, cosmetic acceptability and satisfaction among the parents.

Conclusions: Both, the in vitro and in vivo studies, confirmed the good tolerability, skin compatibility and cosmetic acceptability of this new emollient containing lysate of Lactococcus lactis and ectoin. The emollient represents a new approach for restitution of the cutaneous barrier in patients with $\mathrm{AD}$, through the topical application of the lysate of probiotic bacteria, which acts on the epidermis thereby strengthening the skin barrier.

References: Guéniche A et al. Bifidobacterium longum lysate, a new ingredient for reactive skin. Exp Dermatol. 2010;19(8):e1-8.

Conflict of interest: No conflicts of interest.

\section{$309 / 65$}

EFFICACY OF PROBIOTICS AGAINST ACUTE DIARRHOEA IN WARM AREAS

\author{
${ }^{(1)}$ C. Jíménez-Rodríguez; ${ }^{(2)}$ J. Jiménez-Díaz; ${ }^{(3)}$ F. Hernández-Martínez; \\ ${ }^{(2)} B$. Rodríguez-de-Vera. \\ (1)Farmaceútica Colegio Oficial de Farmaceúticos de Las Palmas. \\ Las Palmas de Gran Canaria.; ${ }^{(2)}$ Departamento Enfermería \\ Universidad de Las Palmas de Gran Canaria.; ${ }^{(3)}$ Servicio \\ Prevención Riesgos Laborales.Cabildo de Lanzarote. Arrecife de \\ Lanzarote.
}

Introduction: Acute diarrhoea is a public health problem and probiotics as adjuvant treatment have shown effectiveness in the prevention and treatment with Lactobacillus casei or Sacharomyces boulardii, since they have been shown to decrease the duration and frequency of acute diarrhoea.

Objective: To describe the effectiveness of the use of probiotics in children under 10 years of age that suffer from heat diarrhoea on the basis that their intake can modify the composition of the microbiota and act against enteric pathogens.

Methods: Randomized double blind study in a paediatric consultation in the Canary Islands with 40 children, between 5 and 10 years old, who has suffered acute diarrhoea. The experimental group was treated with Lactobacillus for 7 days and the control group with a placebo at the same time. Data collection period July-September 2017. Informed consent was obtained from parents or guardians.

Results: Average of age 7.35 years $(n=40)$. By age group, 8 years old children were the most frequently recruited age slot in the whole cohort of acute diarrhoea (37.5\%). In the group that took Lactobacillus, a reduction in episodes of diarrhoea was found in $18 \%$ more than in the placebo group. When compared with placebo, it was shown that there was a reduction in the duration of diarrhoea by intake of probiotics 72 hours as compared to 120 hours in placebo group.

Conclusions: In the group that took Lactobacillus, a reduction in episodes of diarrhoea was found in $18 \%$ more than in the placebo group the intake of probiotics.

References: -Álvarez Calatayud G, Apiroz F. Empleo de probióticos y prebióticos en atención primaria. Nutr Hosp 2015; 31(1): 59-63.

-Manrique Vergara D, Carreras Ferrer I, Ortega Annló E, González Sánchez ME. Probiòticos; más allá de la salud intestinal. Nutr hosp 2014; 30(2):63-67.

Conflict of interest: Authors declare no conflict of interest. 


\section{9/72}

\section{GENERAL POPULATION KNOWLEDGE ASSESS- MENT ON PROBIOTICS}

\author{
${ }^{(1)} C$. Benavent Nuñez; ${ }^{(2)} C$. Núñez Martín; ${ }^{(3)} P$. Callejo Carratalá; ${ }^{(4)} P$. \\ Zubiaur Precioso. \\ (1)Farmacéutico adjunto. Farmacia Núñez Martín. Madrid.; \\ (2)Farmacéutica titular. Farmacia Núñez Martín. Madrid.; \\ ${ }^{(3)}$ Farmacéutica adjunta. Farmacia Núñez Martín. Madrid.; \\ ${ }^{(4)}$ Alumno de doctorado. Hospital Universitario de la Princesa. \\ Madrid.
}

Introduction: There is a growing demand from Spanish population regarding products containing probiotics. The evidence of efficacy on treatment and prevention of different pathologies, concerning gastrointestinal and vaginal fields, has created an emerging development of these products on pharmacies.

Objectives: The objective of this study was to assess the knowledge on probiotics of non-professional population who came to a pharmacy.

Methods: A questionnaire with open and close questions has been completed by 180 adult patients, randomly chosen among those who came to our pharmacy for any kind of consultation during the months of April and June of 2017.

Results: $46 \%$ of the patients did not identify probiotics (by giving a personal definition). $77 \%$ of the patients who did identify these products have used them at least once.

$40 \%$ of the patients have consumed probiotics in the last 6 months, $23 \%$ during last year, $36 \%$ more than one year ago and $4 \%$ did not remember.

Regarding uses, probiotics were mainly consumed as complementary treatment of antibiotic therapy (41\%), digestive complaints $(26 \%)$, diarrhoea (22\%), constipation (5\%) and other pathologies (6\%).

In $53 \%$ of the cases, probiotics were prescribed by doctors; $38 \%$ recommended by pharmacists; $8 \%$ used following a friend or relative's advice and $1 \%$ of patients consulted the internet.

$41 \%$ of the patients regard probiotics as very effective, $44 \%$ considered them useful, $11 \%$ little effective and only $4 \%$ of patients manifested not to have noticed any results.

$84 \%$ of the users would recommend it.

Conclusions: In conclusion, probiotics' divulgation by sanitary professionals among non-professional population is required in order to get better results and further applications. However, a highly satisfactory perception of these products from users has been shown.

References: Microbiota autóctona, probióticos y prebióticos. Abordaje en Farmacia comunitaria. SEFAC.

Conflict of interest: The present work has NOT received any partial or total financing, from any corporation with economic interest on these products.

\section{9/73 \\ BENEFICIAL EFFECTS OF A PROBIOTIC MIXTURE IN ADULT PATIENTS WITH ACUTE DIARRHEA}

\author{
A. Vilaseca. \\ Pharmacist. General Manager PILEJE SLU. Barcelona. Spain.
}

Introduction: Probiotics have well-known beneficial effects on diarrhoea. However, benefits have to be substantiated by experimental evidence because probiotic effects tend to be strain specific. We previously reported that a mixture of three strains of lactic acid bacteria and one strain of bifidobacteria (B. longum LA 101, L. helveticus LA 102, L. lactis LA 103 and S. thermophilus LA 104) was strongly antidiarrhoeic through the combination of antimotility and antisecretory properties in the castor oil-induced diarrhea model in rats ${ }^{1}$. Observations were also in favor of an antinociceptive effect.

Objetive: The objective of the present study was to assess the benefits of the probiotic mixture in patients with acute diarrhoea.

Methods: This was a descriptive prospective observational study performed in adult patients with acute diarrhoea ( $>3 \mathrm{soft}$ stools/day or at a frequency considered abnormal by the subject) that took one dose of the mixture $\left(30 \times 10^{9}\right.$ bacteria) per day during 4 days. Patients had to complete a questionnaire before, during and after supplementation. The main objective was to describe the evolution of stool consistency (Bristol scale) over 5 days; stool number, abdominal pain, flatulence, bloating, tiredness, nausea, and vomiting were also assessed.

Results: Data from 34 patients were analysed. Normalization of stool consistency and frequency occurred after two days of supplementation. There was also a rapid decrease in abdominal pain (within two days) whereas bloating and tiredness symptoms disappeared more progressively. No side effects were reported.

Conclusions: These results suggest that the probiotic mixture tested could relieve effectively acute diarrhoea and associated symptoms.

References: Holowacz, S, et al. Antidiarrheal and antinociceptive effects of a probiotic mixture in rats. J Prob Health 2016 ; 4(3): 155. doi: 10.4172/2329-8901.1000155.

Conflict of interest: No side effects were reported. 


\section{9/74}

\section{PROMOTION OF NURSING COMPETENCE IN THE USE AND MANAGEMENT OF PROBIOTICS FROM THE PROFESSIONAL COLLEGE}

\author{
${ }^{(1)} N$. Costanzo Usan; ${ }^{(2)} R$. Menezo Viadero; ${ }^{(3)}$ A. Domínguez \\ Santamaría; ${ }^{(4)} R$. Cardeñoso Herrero; ${ }^{(5)} A$. Gualdrón Romero. \\ ${ }^{(1)}$ Nurse. Surgical Area of the University Hospital Marques \\ of Valdecilla. Public Service Health of Cantabria. Cantabria. \\ Spain.; ${ }^{(2)}$ Nurse. Cazoña Heath Center. Public Service Health of \\ Cantabria. Cantabria .Spain.; ${ }^{(3)}$ Nurse. Altamira Health Center. \\ Public Service Health of Cantabria. Cantabria .Spain.; ${ }^{(4)}$ Nurse. \\ Cudeyo Health Center. Public Service Health of Cantabria. \\ Cantabria. Spain.; ${ }^{(5)}$ Nurse. Sierrallana Hospital . Public Service \\ Health of Cantabria. Cantabria. Spain.
}

Introduction: Considering all the benefits that probiotics and prebiotics can contribute, the short scientific research about it, and the deficit formation of the nurses in this area, makes necessary to advance the research and investigation through the Official Nurses College in this new componential area.

For such reason, we need to incorporate the use of them in the nurses daily practice with preventive actions and complementary treatments in chronicle diseases associate to dysbiosis.

Objectives: To promote research and training at the competence area in uses and indications of prebiotics and probiotics within nurses profession.

Methods: Observational, descriptive and analytic method through conglomerate to improve training and investigation about use, handling, and indication on probiotics and prebiotics.

The Official Nurses College promotes the constitution of works groups to carry out this project started on 2016, through the integrative nutrition approach, on the context of the chronicle pathology diseases, giving therapeutic diet patterns on the use and management of probiotics and prebiotics supplementation

Results: They were formed 470 nurses in 4 courses, 5 workshops, and 1 working day. This is the $20 \%$ of the total of Cantabria's nurses

Conclusions: The integral approach of the prevention and the chronicle pathology treatment makes necessary the incorporation of prebiotics and probiotics with adequate formation of all the professionals. At the same time promote scientific method increases the knowledge in this competencies area so important to all Spanish nurses

References: - Peláez C., Requena T. La microbiota Intestinal. Ed. La Catarata; 2017.

- Enders G. La digestión es la cuestión.: Ed. Urano; 2015.

- Guarner F. Papel de la flora intestinal en la salud y enfermedad. Nutr Hosp. 2007

- Schoffro M. El milago probiotico. Ed. Grijalbo.2016.

- Formative web www.elprobiotico.com.

- Formative web www.gutmicrobiotaforhealth.com

Conflict of interest: The authors declare not to have interest conflicts.

\section{$309 / 81$ \\ THE EFFICACY, SAFETY, AND TOLERABILITY OF PROBIOTICS ON SYMPTOMS OF DEPRESSION: RESULTS FROM A PILOT STUDY}

\author{
${ }^{(1)}$ C. Wallace; ${ }^{(2)}$ J. Foster; ${ }^{(3)}$ R. Milev. \\ (1)PhD Candidate. Centre for Neuroscience Studies. Department \\ of Psychiatry. Providence Care Hospital. Queen's University. \\ Canada.; ${ }^{(2)}$ Department of Psychiatry and Behavioural \\ Neurosciences. McMaster University. Hamilton. Canada.; ${ }^{(3)}$ Centre \\ for Neuroscience Studies. Queen's University. Kingston. Canada.
}

Introduction: Preclinical and clinical studies have shown that consuming a probiotic supplement can improve mood, anxiety, and cognition in rodents and healthy humans [1]. Evidence shows that these outcomes may be driven by probiotics reducing overall levels of inflammatory markers and increasing serotonin availability, a neurotransmitter depleted in depression [2]. However, the effects of probiotics have yet to be tested in depressed patients.

Objectives: The aim of this 8-week, open-label pilot study was to examine clinical changes in depressive symptoms before and after the introduction of a probiotic supplement in 10 treatment-naïve participants diagnosed with major depressive disorder (MDD).

Methods: Participants recruited from the Kingston area via paper and online advertisements orally consumed a probiotic supplement once daily containing Lactobacillus helveticus R0052 and Bifidobacterium longum R0175 (Probio'Stick; Lallemand Health Solutions). Mood, anhedonia, anxiety, and sleep quality were assessed using validated clinical scales; sleep was also assessed objectively using a polysomnogram. To examine the full composition of the microbiome, fecal samples were collected and analyzed using $16 \mathrm{~S}$ rRNA sequencing. Further, blood samples were collected to examine changes in plasma levels of inflammatory markers, tryptophan, and serotonin.

Results: The study found significant improvements in overall mood, anhedonia, and anxiety after just 4 weeks of the probiotic intervention, and significant improvements in subjective sleep quality after 8 weeks. Objective sleep quality was not affected. Results for biological measures are not yet available.

Conclusions: These findings suggest a role for probiotics in alleviating depressive symptoms in humans. However, additional evidence is required to substantiate these claims. The data from this pilot study has been used to plan a double-blind randomized placebo-controlled trial to further assess these effects.

References: [1] Wallace, CJK et al. (2017). Ann Gen Psychiatry

[2] Dinan et al. (2011). Aust N Z J Psychiatry

Conflict of interest: Declaration: This study was funded by Lallemand Health Solutions. 


\section{$309 / 82$ \\ ORAL PROBIOTIC REDUCES PAIN AFTER THIRD MOLAR EXTRACTION PROCEDURE}

\author{
${ }^{(1)}$ R. Pons Calabuig; ${ }^{(2)}$ E. Ferrés Amat; ${ }^{(2)}$ J. Mareque Bueno; ${ }^{(3)} E$. \\ Navarro Tapia; ${ }^{(3)}$ E. Astó Sanchez-Lafuente; ${ }^{(4)}$ J. Espadaler Mazo; ${ }^{(2)} \mathrm{E}$. \\ Ferrés Padró. \\ (1)Department of oral and maxillofacial surgery. Faculty of \\ Dentistry. International University of Cataluña. Barcelona. Spain.; \\ ${ }^{(2)}$ Service of oral and maxillofacial surgery. Fundacio Hospital de \\ Nens de Barcelona. Barcelona. Spain.; ${ }^{(3)}$ Innovation department. \\ AB-Biotics S.A. Barcelona. Spain.; ${ }^{(4)}$ AB-Biotics S.A. Barcelona. \\ Spain.
}

Introduction: The oral microbiota contains hundreds of bacterial species [1], which can influence physiological processes of recovery and the risk of complications after tooth extraction procedures.

Objetive:To evaluate the impact of an oral probiotic on clinical parameters after tooth extraction.

Methods: Double-blind, randomized, clinical trial, comparing probiotic (AB-Dentalac, AB-Biotics $\mathrm{SA}$ ) to placebo oral tablets, twice daily for 7 days, in subjects undergoing a third molar extraction. Exclusion criteria included chronic disease, current antibiotic or probiotic use, and toxic habits. Subjects rated each of swelling, eating difficulty and pain in a $0-10$ scale, daily. Extraction site infection was assessed by specialists 1 week post-extraction. Normality was assessed by Shapiro-Wilk test. Continuous data was compared using Mann-Whitney's test and proportions using Fisher test.

Results: 20 subjects were randomized to probiotic and 17 to placebo. No differences were observed in drop-out rate $(n=3$ probiotic, $n=4$ placebo) or infection rate $(n=2$ probiotic, $n=2$ placebo). Subject's diary recovery was low, without differences among groups ( $n=12$ probiotic, $n=9$ placebo). Per-protocol $(n=21)$, swelling and pain scores were comparable among groups $(\mathrm{p}>0.10)$ at baseline (day 1), as were age, gingival index and gender distribution, but eating difficulty score was marginally higher in the probiotic group $(\mathrm{p}<0.05)$. Reductions vs. day 1 in pain score and eating difficulty score were larger in probiotic group than placebo on days 5, 6 and 7 ( $\mathrm{p}<0.05)$. No other significant differences were observed.

Conclusions: After third molar extraction, oral probiotic helped achieve a larger reduction in pain towards the end of the first week.

References: Samaranayake, L., Matsubara, V.H. Normal Oral Flora and the Oral Ecosystem. Dent Clin N Am 61 (2017) 199-215

Conflict of interest: $\mathrm{EN}$ and JE are full-time employees of AB-BIOTICS SA. JMB has acted as consultant of AB-BIOTICS SA. Other authors report no conflicts of interest.

\section{Track 3: Microbiology}

\author{
Reviewers: \\ Abelardo Margolles Barro \\ Institute of Dairy Products of Asturias, IPLA-CSIC. Oviedo.
}

Alfonso Clemente Gimeno

Experimental Station of Zaidín (EEZ-CSIC). Granada.

Juan Evaristo Suárez Fernández

Oviedo University. Asturias.

Teresa Requena Rolanía

Research Institute in Food Sciences, CIAL (CSIC- UAM). Madrid.

Juan Miguel Rodríguez Gómez

Veterinary School. Universidad Complutense de Madrid.

\section{Gaspar Pérez Martínez}

Institute of Agrochemistry and Food Technology, IATA-CSIC.

Valencia.

\section{$309 / 3$ \\ MICROBIOME CORE IN PATIENTS WITH RELAPS- ING-REMITTING MULTIPLE SCLEROSIS}

\begin{abstract}
${ }^{(1)}$ V. Navarro-López; ${ }^{(2)}$ JM. Mingot; ${ }^{(3)}$ M. Carrion-Gutierrez; ${ }^{(3)} Y$. PayáEspinosa; ${ }^{(4)} B$. Ruzafa Costa; ${ }^{(5)}$ E. Núñez Delegido.

${ }^{(1)}$ Clinical Microbiology and Infectious Disease Unit. Hospital Universitario Vinalopo. Elche. Department of Clinical Medicine. MiBioPath Group. Universidad Catolica San Antonio de Murcia. Murcia. Spain.; ${ }^{(2)}$ Korott S.L. Alcoy, Alicante, Spain; ${ }^{\left({ }^{(3)} \text { Department }\right.}$ of Clinical Medicine. MiBioPath Group. Universidad Catolica San Antonio de Murcia. Murcia. Spain.; ${ }^{(4)}$ Universidad Católica San Antonio de Murcia. Grupo MiBioPath. Murcia. Spain.; ${ }^{(5)}$ Institute of Bioengineering, University Miguel Hernández, Alicante, Spain
\end{abstract}

Introduction: Recent findings indicate the effect of gut microbiome on certain neurological diseases such as multiple sclerosis (AD). In this study we attempt to reveal the microbiome composition in a case series of Caucasian population with relapsing-remitting multiple sclerosis (RRMS).

Objectives: To describe the gut microbiome in a case series of Caucasian population wit RRMS, comparing these results with the gut microbiome in healthy people. We also describe bacteria form groups (clusters) according to their similarity amongst individuals.

Methods: Patients with RRMS were included in the study. Massive genome sequencing of stool samples of patients was performed. DNA from stool samples was isolated following the protocols of Yuan and coworkers with minor modifications, with the aid of MagnaPure Compact System (Roche Life Science). The microbiome of a cohort of 16 patients with remittent recurrent active sclerosis multiple was obtained by 16 s rRNA massive sequencing with MiSeq platform (Illumina inc, San Diego).

Results: Microbiome in 17 patients with RRMS was obtained. We have a mean of 112 different species of bacteria in each patient. In our series the most prevalent bacteria are Bacteroides. Most

9th Workshop SEPyP 
important differences when comparing these microbiomes with healthy population are related to the bacteria Prevotella and Streptococcus sp. We also assessed bacteria clustering among our cohort of individuals (Figure; during presentation).

Conclusions: There is a clear difference in the microbiota of patients with RRMS compared with the healthy population. Difference affects to the genera Prevotella and Streptococcus, that appears decreased in the group with relapsing-remitting multiple sclerosis when compared with the group of healthy people

References: Hindson J. Multiple sclerosis: A possible link between multiple sclerosis and gut microbiota. Nat Rev Neurol. 2017 Sep 29. doi:10.1038/nrneurol.2017.142

Rowin J, Xia Y, Jung B, Sun J. Gut inflammation and dysbiosis in human motor neuron disease. Physiol Rep.2017 Sep;5(18)

Conflict of interest: No conflict of interest

\section{$309 / 7$ \\ PROTEOMIC ANALYSIS OF LACTOBACILLUS PEN- TOSUS UNDER BILE STRESS}

\begin{abstract}
${ }^{(1)}$ H. Abriouel Hayani; ${ }^{(1)}$ B. Pérez Montoro; ${ }^{(1)}$ N. Caballero Gómez; ${ }^{(2)}$ S. Ennahar; ${ }^{(3)}$ P. Horvatovich; ${ }^{(4)}$ C. Knapp; ${ }^{(1)}$ A. Gálvez; ${ }^{(1)}$ N. Benomar EI Bakali.

(1)Area of Microbiology. Department of Health Sciences. Faculty of Experimental Sciences. University of Jaén. Jaén. Spain.;

${ }^{(2)}$ Analytical Chemistry Team of Bio-active Molecules. UMR ${ }^{(7)(1)(7)(8)}$. IPHC-DSA. University of de Strasbourg. France.; ${ }^{(3)}$ Department of Analytical Biochemistry. Centre for Pharmacy. University of Groningen. Groningen. The Netherlands.; ${ }^{(4)}$ Department of Civil and Environmental Engineering. University of Strathclyde. Glasgow. Scotland. United Kingdom.
\end{abstract}

Introduction: Naturally fermented Aloreña green table olives are potential source of probiotic Lactobacillus pentosus which were isolated throughout fermentation process. These strains exhibited high tolerance to low $\mathrm{pH}$ and high concentrations of bile salts as well as antimicrobial activity against several pathogens (Abriouel et al., 2012).

Objectives: To determine the biomarkers for bile tolerance in potential probiotic L. pentosus strains using comparative proteomic analysis.

Methods: In this study, proteomic analysis of L. pentosus strains exhibiting three phenotypes (resistant, intermediate and sensitive) of bile tolerance was done under standard conditions and under bile stress conditions using two-dimensional electrophoresis (2-DE), tryptic digestion, liquid chromatography-mass spectrometry analysis and database search for protein identification (Hamon et al., 2011).

Results: Comparative proteomic analysis of $L$. pentosus strains: L. pentosus 5C3 (resistant), L. pentosus CF2-15P (intermediate) and $L$. pentosus $5 \mathrm{C} 2$ (sensitive) revealed qualitative and quantitative differences in proteomes being 14 proteins differentially expressed. Among these proteins, 2,3-bisphosphoglycerate-dependent phosphoglycerate mutase and ATP-dependent

9th Workshop SEPyP 6-phosphofructokinase may be the key in the bile salt response and adaptation in L. pentosus. These two proteins are involved in carbohydrate metabolism which increases the production of energy-rich intermediates such as ATP and NADH for the survival of L. pentosus under stress conditions.

Conclusions: These results showed that comparative proteomic analysis allowed to identify bacterial bile tolerance biomarkers for the preliminary selection of strains with the best probiotic potential.

References: Abriouel, H., Benomar, N., Cobo, A., Caballero, N., Fernández Fuentes, M. Á., Pérez-Pulido, R., Gálvez, A., 2012. Characterization of lactic acid bacteria from naturally-fermented Manzanilla Aloreña green table olives. Food Microbiol. 32, 308-316.

Hamon, E., Horvatovich, P., Izquierdo, E., Bringel, F., Marchioni, E., Aoude-Werner, D., Ennahar, S., 2011. Comparative proteomic analysis 530 of Lactobacillus plantarum 531 or the identification of key proteins in bile tolerance. BMC Microbiol. 11, 63.

Conflict of interest: No conflict of interest.

\section{9/17 \\ OUTER MEMBRANE VESICLES AND SOLUBLE FACTORS SECRETED BY PROBIOTIC AND COM- MENSAL E. COLI STRAINS: EFFECT ON SALMO- NELLA ENTERICA SEROVAR TYPHIMURIUM IN- FECTION}

\author{
${ }^{(1)}$ M. Fábrega Fernández; ${ }^{(1)}$ M. Cañas Pachecho; ${ }^{(1)}$ N. Díaz Garrido; \\ ${ }^{(1)} J$. Badía Palacín; ${ }^{(1)}$ R. Giménez Claudio; ${ }^{(1)}$ L. Baldomà Llavinés; ${ }^{(2)} A$. \\ Elulalio. \\ (1)Departamento de Bioquímica y Fisiología. Sección Bioquímica \\ y Biología Molecular. Instituto de Biomedicina. Universidad de \\ Barcelona. Barcelona.; ${ }^{(2)}$ Institute for Molecular Infection Biology. \\ University of Würzburg. Germany.
}

Introduction: In the last years, increasing evidences have demonstrated that the gut microbiota, which includes probiotic and commensal strains, play a crucial role in host resistance against invading pathogens within the intestine. The Gram-negative probiotic Escherichia coli Nissle $1917(\mathrm{EcN})$ has been widely studied as antagonist of enteropathogens such as Salmonella spp., albeit no clear consensus has been reached on what the mechanism of action is.

Objectives: In this work, we analysed the effect of secreted factors released by EcN on Salmonella infection, a pathogen with high impact on health society. In parallel, other non-pathogenic E. coli strains (ECOR12, ECOR63, DH5a and MG1655) were used for comparison.

Methods: OMVs and supernatants from E. coli strains were isolated to host cell stimulations directly, or using a transwell system. Intracellular Salmonella was assayed by CFU assay and confocal microscopy. Moreover, secreted Salmonella proteins were isolated by TCA precipitation.

Results: Results showed that supernatants from both probiotic and commensal E. coli strains reduce Salmonella invasion, without affecting Salmonella epithelial cell-adhesion or growth. We 
demonstrated that this effect is not due to outer membrane vesicles (OMVs) released by EcN, but to other secreted components. Enzymatic treatment confirmed that the active secreted factor is not a protein, DNA or RNA molecules. We also observed that EcN supernatant slightly reduces secretion of Salmonella invasion proteins.

Conclusions: In conclusion, this work suggests that secreted factors might diminish Salmonella invasion by interfering with the T3SS secretion system or by modulating the expression of regulatory genes involved in Salmonella invasion. This effect seems not to be $\mathrm{EcN}$ specific but common to non-pathogenic E. coli strains.

References: - Altenhoefer et al. (2004)

- Schierack, P. et al. (2011)

- Alvarez, C.-S. et al. (2016)

- Kleta, S. et al. (2006)

Conflict of interest: Authors declare no conflict of interest.
$40 \mathrm{~kb}$ plasmid designated pPE3. Adhesion of CUPV1411 strain to enterocytes was superior to that of its isogenic strain and the pattern of immunomodulation of THP-1 cells by the 2 -substituted $(1,3)-\beta$-D-glucan suggested an anti-inflammatory potential of this polymer.

Conclusions: Cider-isolated Pediococcus ethanolidurans CUPV1411, produces one HePS and a $(1,3)-\beta$-D-glucan substituted in O-2 position. The latter seems to confer probiotic properties to this strain, and so, it could be used in the development of functional food.

References:

1. Pérez-Ramos et al. ISBN: 9780128021897

2. Laine et al. (1972). Methods Enzymol 28, 159-167.

3. Fernández de Palencia et al. (2008). Eur Food Res Technol 227, 1475-1484.

4. Chanput et al. (2010). Food Funct 1, 254-261.

Conflict of interest: Authors declare no conflict of interest.

\section{$309 / 23$ \\ PEDIOCOCCUS ETHANOLIDURANS CUPV1411: A CIDER-ISOLATED, FIRST-DESCRIBED STRAIN OF P. ETHANOLIDURANS PRODUCING B-D-GLUCAN}

\author{
${ }^{(1)}$ M. Llamas Arriba; ${ }^{(2)}$ A. Pérez-Ramos; ${ }^{(3)}$ A. Prieto; ${ }^{(2)}$ P. López; ${ }^{(4)} M$. \\ Dueñas Chasco. \\ (1)Department of Applied Chemistry. University of Basque \\ Country. San Sebastián. Department of Molecular Microbiology \\ and Infection Biology. Biological Research Centre (CIB). Madrid. \\ Spain.; ${ }^{(2)}$ Molecular Microbiology and Infection Biology. \\ Biological Research Center. Madrid. Spain.; ${ }^{(3)}$ Department of \\ Biotechnology for Lignocellulosic Biomass. Biological Research \\ Centre (CIB). Madrid. Spain.; ${ }^{(4)}$ Department of Applied Chemistry. \\ University of Basque Country. San Sebastián. Spain.
}

Introduction: Lactic acid bacteria can produce a wide variety of exopolysaccharides (EPSs), and, specifically, cider-isolated LAB can synthesize an O-2 substituted (1,3)- $\beta$-D-glucan (1).

Objectives: Characterization of the EPSs produced by Pediococcus ethanolidurans CUPV1411, a species never before isolated from cider, and its isogenic strain, obtained by chemical mutagenesis. Analysis of the gene encoding the enzyme responsible for the production of the $\beta$-D-glucan, the GTF glycosyltransferase. Evaluation of probiotic and prebiotic properties.

Methods: EPSs isolation was performed. Determination of the molecular weight of the EPSs was carried out by HPLC-SEC and determination of the composition of monosaccharides and O-glycosidic linkages by GC-MS (2). Southern hybridization was used to determine the genomic localization of the $g t f$ gene. Finally, Caco- 2 cell line was used to evaluate the bacterial adhesion capacity (3), and THP-1 human monocytes cell line gave an insight of the immunomodulatory ability (4) of the produced $\beta$-glucan.

Results: $P$. ethanolidurans CUPV1411 synthesizes a $\beta$-D-glucan and an hetropolysaccharide, composed of glucose, galactose, $\mathrm{N}$-acetyl-glucosamine and glycerol-3-phosphate. Its isogenic strain synthesized the same $\beta$-D-glucan, but in a lower amount. Southern hybridization revealed that the $g t f$ gene is located in a

\section{$309 / 24$ \\ TWO INFANT-ISOLATED BIFIDOBACTERIA CON- TAINING POTENTIAL PROBIOTIC PROPERTIES}

\author{
${ }^{(1)}$ M. Llamas-Arriba; ${ }^{(2)}$ Á. Peirotén; ${ }^{(3)}$ A. Puertas; ${ }^{(4)}$ A. Prieto; ${ }^{(5)}$ P. López; \\ ${ }^{(6)}$ E. Rodríguez; ${ }^{(3)}$ M. Dueñas Chasco. \\ (1)Department of Applied Chemistry. University of Basque \\ Country. San Sebastián. Department of Molecular Microbiology \\ and Infection Biology. Biological Research Centre (CIB). Madrid. \\ Spain.; ${ }^{(2)(2)}$ Depatmet of Food Technology. Research and Agrarian \\ Technology National Centre (INIA). Madrid. Spain.; ${ }^{(3)}$ Department \\ of Applied Chemistry. University of Basque Country. San Sebastián. \\ Spain.; ${ }^{(4)}$ Department of Biotechnology for Lignocellulosic Biomass. \\ Biological Research Centre (CIB). Madrid. Spain.; ${ }^{(5)}$ Molecular \\ Microbiology and Infection Biology. Biological Research Center. \\ Madrid. Spain.; ${ }^{(6)}$ Depatmet of Food Technology. Research and \\ Agrarian Technology National Centre (INIA). Madrid. Spain.
}

Introduction: Bifidobacteria predominate in the human gastrointestinal tract. They have a long history of safely use in food and as probiotics. Some of them produce mucous polymers called exopolysacharides (EPSs), responsible for some healthy benefits to the host.

Objectives: Evaluation of the probiotic and technological properties of two bifidobacteria isolated from infant faeces, Bifidobacterium longum INIA P132 and Bifidobacterium infantis INIA P731, and characterization of the EPSs produced by them.

Methods: EPSs isolation was performed. Characterization of EPSs: determination of the molecular weight by HPLC-SEC, analysis of the configuration of the anomeric carbon by FT-IR and determination of monosaccharides composition and O-glycosidic linkages by GC-MS. The adhesion capacity of the two bacteria and their immunomodulation ability were analysed in vitro using culture cell-lines: Caco-2 human enterocytes for the former, and THP-1 human monocytes for the latter. Finally, another immunomodulation assay was carried out in vitro, using a DSS induced enterocolitis zebra fish model. 
Results: Both strains showed moderate resistance to gastrointestinal conditions. Moreover, the P132 strain grew in milk better and had tolerance to freezing and freeze-drying. The P731 strain demonstrated a higher adhesion capacity to human Caco- 2 cells than the probiotic bifidobacteria BB12 (1). The EPSs from both bifidobacteria were composed of two fractions with different molecular mass $(\mathrm{Mw})$, in the range of $10^{\wedge} 6$ and $10^{\wedge} 4 \mathrm{Da}$ for $B$. longum and $10^{5}$ and $10^{4} \mathrm{Da}$ for $B$. infantis. Both bacteria produce heteropolysaccharides composed of rhamnose, galactose and glucose. The two EPSs preparations were able to immunomodulate human PMA-THP-1 macrophages exerting an anti-inflammatory effect. In addition, these EPSs were able to reduce larva mortality.

Conclusions: Strains P132 and P731 showed interesting technological properties, so both bifidobacteria and their heteropolysaccharides are potential candidates for the production of functional food.

References: Fernández de Palencia et al. (2008). Eur Food Res Technol 227, 1475-1484.

Conflict of interest: Authors declare no conflict of interest.

\section{$309 / 28$ \\ PROBIOTIC FUNCTIONAL FOODS FOR MALNOUR- ISHED CHILDHOOD POPULATIONS: PROINFANT PROJECT}

\author{
${ }^{(1)}$ P. Ruas Madiedo; (2) R. Aznar; ${ }^{(2)}$ P. Proinfant Consortium. \\ (1)Department of Microbiology and Biochemistry of Dairy \\ Products. Instituto de Productos Lácteos de Asturias (IPLA-CSIC). \\ Villaviciosa. Asturias. Spain.; ${ }^{(2)}$ Instituto de Productos Lácteos \\ de Asturias. Consejo Superior de Investigaciones Científicas. \\ Asturias.
}

Introduction: The concept of "Functional Food" arises in developed countries in which consumers demand foods that, in addition to their nutritional qualities, promote better health and well-being or reduce the risk of disease. In the context of populations suffering from chronic nutritional deficiencies, such as certain groups of children from disadvantaged social strata, the development of a functional food will, on the one hand, alleviate the lack of nutrients if the native raw material selected is rich in macro- and micro-nutrients and, on the other hand, to improve the state of health by including beneficial ingredients.

Objectives: The objective to the ProInfant project is to develop vegetable-based probiotic foods to alleviate chronic nutritional deficiencies and health problems in disadvantaged Iberoamerican children populations.

Methods: To achieve this, 73 researchers, organized in 9 groups from 7 Iberoamerican countries (Argentina, Colombia, España, Guatemala, México, Nicaragua and Perú) together with 2 Italian associated groups, will work in the ProInfant project funded by CYTED organization (Ciencia y Tecnología para el Desarrollo de Iberoamerica). Two of the 9 groups are Spanish: the Coordinator group (CSIC: IPLA, CIAL and CIB) and the A group (UV and UCM)

9th Workshop SEPyP
Results: Traditional fermented foods from the Latin America will be used to search for autochthonous lactic acid bacteria whose technological, nutritional, safe and probiotic characteristics will be characterized: the ability to produce vitamins, antimicrobial compounds and / or to antagonize human pathogens. A vegetable-based probiotic food will be designed for malnourished child populations; its functionality will be analyzed in vitro, in vivo and through an intervention study with children in Guatemala.

Conclusions: ProInfant also focuses on training qualified human resources allowing that each country can develop safe and high nutritional value probiotic foods, using native bacteria and plants.

References: Referencia proyecto P916PTE0233 (http://www. cyted.org/es/estrategicos)

Conflict of interest: The authors declare that there are no conflicts of interest

\section{$309 / 31$ \\ USE OF WHEY FERMENTED BY LACTIC ACID BACTERIA AS A NATURAL PRESERVATIVE WITH ANTIFUNGAL PROPERTIES}

\author{
${ }^{(1)}$ C. Luz Minguez; ${ }^{(2)}$ L. Izzo; ${ }^{(3)}$ V. Lanni; (1)J. Mañes Vinuesa; ${ }^{(1)}$ G. Meca. \\ (1)Laboratory of Food Chemistry and Toxicology. Faculty of \\ Pharmacy. University of Valencia. Spain.; ${ }^{(2)}$ Laboratory of Food \\ Chemistry. Department of Pharmacy. University of Napoles \\ "Federico II". Italy.; ${ }^{(3)}$ Laboratory of Pharmaceutical Chemistry. \\ Department of Pharmacy and Biotechnology. University of \\ Bolonia "Alma Mater Studiorum". Italy .
}

Introduction: Bioconservation is a biotechnological application consisting of the use of microorganisms or their metabolic products in foods in order to inhibit microbial growth, with the aim of improving food safety and extending the shelf life of food products $^{1}$. Food deterioration caused by toxigenic fungi is one of the challenges of food security. In this context, some strains of lactic acid bacteria (LAB) are capable of producing antifungal compounds during fermentation processes.

\section{Objectives: -}

Methods: Four strains of BAL obtained from the Spanish Type Culture Collection (CECT) were used to ferment goat's milk whey (MW) for $72 \mathrm{~h}$ at $37^{\circ} \mathrm{C}$. An antifungal activity study of fermented MW in solid medium was then performed against a group of thirty toxigenic fungi belonging to the genus Fusarium, Penicillium and Aspergillus. The minimum inhibitory concentration (MIC) and the minimum fungicidal concentration (MFC) of each of the fermented MW were also determined in liquid medium.

Results: The results of the study of antifungal activity evidenced that LAB fermented MW inhibited fungal growth in solid medium against different fungal species and presented in a liquid medium a MIC and MFC range of 2-250 g serum of lyophilized fermented milk / L. The lowest MIC and MFC values of LAB fermented whey for the fungi tested were obtained by the strain Lactobacillus plantarum CECT 223. 
Conclusions: The use of natural ingredients obtained from subproducts from the dairy industry and the use of BAL may be a good tool to promote food safety and reduce the massive use of synthetic additives.

References: [1] Crowley, S., Mahony, J., \& van Sinderen, D. (2013). Current perspectives on antifungal lactic acid bacteria as natural bio-preservatives. Trends in Food Science \& Technology, 33, 93-109.

Conflict of interest: Authors declare no conflict of interest.

\section{$309 / 33$ \\ IN VITRO STUDY OF THE PROBIOTIC POTENTIAL OF MICROORGANISMS CONTAINED IN FOOD SUPPLEMENTS AND MEDICINES}

\author{
${ }^{(1)}$ A. Llatas Mateu; ${ }^{(2)}$ C. Luz Minguez; ${ }^{(3)} M$. Werner; ${ }^{(3)}$ F. Bittencourt \\ Luciano; ${ }^{(2)}$ J. Mañes Vinuesa; ${ }^{(2)} G$. Meca. \\ (1)Laboratory of Food Toxicology. Faculty of Pharmacy. University \\ of Valencia. Spain.; ${ }^{(2)}$ Laboratory of Food Chemistry and \\ Toxicology. Faculty of Pharmacy. University of Valencia. Spain.; \\ ${ }^{(3)}$ Department of Animal Science. Life Science School. Pontifical \\ Catholic University of Paraná. Brazil.
}

Introduction: Probiotics are living microorganisms that after being administered in adequate amounts, can confer health benefits on the host beyond those inherent to basic nutrition. A good probiotic should meet certain criteria such as surviving in the intestinal environment (resisting both $\mathrm{pH}$ of the stomach and bile acids), capacity to adhere to the intestinal epithelium and being non-pathogenic and non-toxic ${ }^{1}$.

Objectives: -

Methods: In the present study, a selection of eleven commercial probiotic strains was characterized by testing; resistance to $\mathrm{pH}$ and bile, resistance to antibiotics, detection of enzymes by API ZYM, antimicrobial activity against pathogens (Escherichia coli, Clostridium perfringens, Staphylococcus aureus, Yersinia enterocolitica, Listeria monocytogenes and Salmonella enterica spp.), cell adhesion and competition adhesion to pathogens (Salmonella enterica spp.) in vitro.

Results: The results of these tests show that probiotics presented good resistance to gastrointestinal $\mathrm{pH}$ and bile conditions, obtaining viability values at $\mathrm{pH} 2.5$ and bile $0.5 \%$ with a range of $4-59 \%$ and $2-56 \%$ respectively. With regard to the antimicrobial activity against pathogens, the eleven probiotics showed activity against a large number of pathogens, especially Escherichia coli, Clostridium perfringens, Salmonella enterica spp. in solid medium. Adhesion studies in Caco-2/TC7 cells evidenced an adhesion of the strains in a range of $2-7 \%$, also eight of these strains had a \% reduction of the adhesion of Salmonella enterica spp. between 1-46\%.

Conclusions: The results of the tests performed in the study indicate that there are significant differences in the characteristics of commercial probiotics. On the other hand, this information can be used as a control in future research focused on the characterization of the probiotic potential of strains isolated from different matrices of interest.
References: 1.Hossain, M.I., Mohammad Sadekuzzaman, M., \& Ha, S.D. (2017). Probiotics as potential alternative biocontrol agents in the agriculture and food industries: A review. Food Research International,100,63-73.

Conflict of interest: Authors declare no conflict of interest.

\section{$309 / 39$}

\section{SHARING OF BACTERIAL STRAINS BETWEEN PRE-COLOSTRUM AND INFANT SALIVA: CUL- TURE INDEPENDENT AND CULTURE DEPEND- ENT APPROACHES}

\author{
${ }^{(1)}$ L. Ruiz Garcia; (2) C. García Carral; ${ }^{(3)}$ A. Boix-Amorós; ${ }^{(3)} R$. \\ Bacigalupe; ${ }^{(4)}$ C. Beatriz; ${ }^{(5)}$ A. Checa; ${ }^{(3)} A$. Mira; ${ }^{(6)} J$. Rodríguez Gómez. \\ (1)Department of Microbiology and Biochemistry of Dairy \\ Products. Dairy Research Institute of Asturias. IPLA-CSIC. \\ Villaviciosa. Spain. Department of Nutrition. Food Science and \\ Food Technology. Complutense University of Madrid. Madrid. \\ Spain; ${ }^{(2)}$ Department of Nutrition. Food Science and Food \\ Technology. Complutense University of Madrid. Probisearch SL. \\ Madrid. Spain.; ${ }^{(3)}$ Department of Health and Genomics. Center \\ for Advanced Research in Public Health. FISABIO Foundation. \\ Valencia. Spain.; ${ }^{(4)}$ Department of Nutrition. Food Science and \\ Food Technology. Complutense University of Madrid. Madrid. \\ Spain.; ${ }^{(5)}$ Maternity Service. Centro de Salud Arrabal. Zaragoza. \\ Spain.; ${ }^{(6)}$ Professor. Dpt. Nutrition. Food Science and Food \\ Technology. Complutense University of Madrid. Spain.
}

Introduction: The infant oral microbiome might impact the infant gut microbiome establishment providing the first commensals that will reach the infant gut(1). Besides, human milk represents a source of bacteria for the initial establishment of the oral (and gut) microbiomes in the breastfed infant $(2,3)$ although little is known on the origin of the human milk microbiome and its impact on the infant oral microbiome.

Objectives: To investigate the maternal precolostrum and infant oral microbiomes to identify bacterial species (strains) shared in the maternal-infant dyad.

Methods: $16 \mathrm{Sr}$ RNA sequencing microbiome profiling was performed on maternal precolostrum and infant saliva collected from 19 mother-infant pairs. Bacteria were cultured, isolated and identified by MALDI-TOF. RAPD-PCR and full genome sequencing was performed on those isolates that, belonging to the same species, were isolated from both the maternal and infant samples in the same mother-infant pair.

Results: Maternal precolostrum and infant salivary microbiomes significantly differed, although certain taxa were consistently shared, Streptococcus and Staphylococcus spp being the most abundant. In 13 pairs, at least one isolate from the same species was recovered from both sample types. From those, isolates obtained from precolostrum and infant saliva in 8 of the pairs could not be distinguished by RAPD-PCR profiling. 8 out of 10 fully sequenced isolate pairs were $>99.9 \%$ identical at nucleotide level, thus sug- 
gesting the sharing of bacteria at strain level between precolostrum and infant oral microbiomes.

Conclusions: The presence of oral bacteria in precolostrum, before contact with the newborn, indicates that they are not a contamination from the infant and suggests that at least some oral bacteria colonize the infant's oral cavity through transmission through breastmilk.

References: 1 PLoS One, 2014, 9(2):e89269

2 Sci Rep, 2017, 7(1):9940

3 Front Microbiol, 2017, 8:1214

Conflict of interest: The authors declare that there are no conflicts of interest

\section{$309 / 40$}

\section{UNVEILING THE IMPACT OF INTRAPARTUM ANTI- MICROBIAL PROPHYLAXIS IN THE EARLY MICRO- BIOME THROUGH METAGENOMICS ANALYSIS}

\author{
${ }^{(1)}$ S. Arboleya Montes; ${ }^{(1)}$ A. Nogacka; ${ }^{(2)}$ C. Milani; ${ }^{(3)}$ N. Fernández \\ González; ${ }^{(3)}$ M. Suárez Rodríguez; ${ }^{(1)} \mathrm{C}$. González de los Reyes \\ Gavilan; ${ }^{(3)}$ G. Solís Sánchez; ${ }^{(2)}$ M. Ventura; ${ }^{(4)}$ M. Gueimonde.
}

\begin{abstract}
(1)Microbiology and Biochemistry Department. Dairy Research Institute of Asturias (IPLA-CSIC). Villaviciosa-Asturias. Spain. Diet. Microbiota and Health Group. Health Research Institute of Principado de Asturias (ISPA). Oviedo. Spain.; (2)Laboratory of Probiogenomics. Department of Life Sciences. University of Parma. Italy:; ${ }^{(3)}$ Diet. Microbiota and Health Group. Health Research Institute of Principado de Asturias (ISPA). Oviedo. Spain.Pediatrics Service Department. Central University Hospital of Asturias (HUCA-SESPA). Oviedo. Spain.; ${ }^{(4)}$ Department of Microbiology and Biochemistry of Dairy Products. Instituto de Productos Lácteos de Asturias (IPLACSIC). Group Diet, Microbiota and Health. Instituto de Investigación Sanitaria del Principado de Asturias (ISPA). Oviedo. Spain.
\end{abstract}

Introduction: The correct establishment of the intestinal microbiome (IM) at the beginning of life plays a key role in infant and later health. Some factors affecting this process have been studied; however, other like the intrapartum antimicrobial prophylaxis (IAP), in spite of been present on over $30 \%$ of total deliveries, have been much less studied. Given the increasingly evident relationship between early microbiota, later disease risk and the rising worries regarding antimicrobial resistances, there is an urgent need to assess the potential adverse consequences of IAP in the establishment of the IM.

Objectives: The objective of this study was to assess the effect of the IAP on the IM composition in full-term 30-days-old infants by metagenomics approach.

Methods: Gut microbial DNA was extracted from faecal samples of nine one-month-old babies, five of which were born from mothers receiving IAP. Trying to avoid possible confounders the recruited cohort of babies was in all cases composed of full-term, vaginally delivered individuals. Illumina shotgun sequencing of the DNA extracted was performed and reconstruction of the microbiomes allowed taxonomic and functional characterization.

9th Workshop SEPyP
Results: In spite of the large inter-individual differences, IAP-exposed babies showed slight microbiota differences when compared with babies which mothers did not received prophylaxis, being the bifidobacteria and bacteriodes populations affected. The functional classification of coding reads by means of different databases allowed to identify differences on the abundance of transporters and enzymes involved in the antibiotic resistance in infants exposed to IAP compared with the not exposed counterparts.

Conclusions: Our results confirm an impact of the IAP treatment on the correct establishment of the IM by using metagenomics analysis and highlight the need of new dietary strategies development based on microbial modulation with the objective to minimize the IAP effect at the early stage of life.

References: -

Conflict of interest: All authors declare that no competing interest exits.

\section{$309 / 45$ \\ STARTERS OF TABLE GREEN OLIVES CAN AF- FECT FUNCTIONAL PROPERTIES OF BRINE EPS}

\author{
${ }^{(1)} Y$. Zhu; ; ${ }^{(2)}$ G. González Ortiz; ${ }^{(3)}$ R. Jiménez Díaz; ${ }^{(4)}$ S. Martín Orúe. \\ (1) Sniba Department of Animal and Food Science University \\ Autonoma de Barcelona. Barcelona.; ${ }^{(2)}$ Servicio de Nutrición \\ y Bienestar Animal. Departament de Ciència Animal i dels \\ Aliments. Universitat Autònoma de Barcelona. Barcelona.; \\ ${ }^{(3)}$ Departamento de biotecnología de alimentos. Instituto de la \\ grasa. Cconsejo superior de investigaciones científicas. Sevilla.; \\ ${ }^{(4)}$ Animal Nutrition and Welfare Service. Animal and Food Science \\ Department. Universitat Autònoma de Barcelona. Barcelona. \\ Spain.
}

Introduction: In this study, a strain of Candida boidinii and four of $L$. pentosus were used as starters in green-table olive fermentation (10 treatments in a 2 x 5 design).

Objectives: A total of 20 exopolysaccharides (EPS) samples were isolated from the brines ( 2 fermentors per treatment) and tested by different miniaturized in vitro models using porcine intestinal epithelial cells (IPEC-J2).

Methods: In vitro studies included an adhesion test (AT) aimed to evaluate the ability of EPS to attach specifically ETEC K88 and three different approaches to evaluate its interference on adhesion of the pathogen to IPEC-J2 cells: competition (CT), exclusion (ET) and displacement tests (CT).

Results: AT demonstrated that most EPS samples could attach specifically ETEC K88 $(\mathrm{P}<0.05)$. Regarding the studies with IPEC-J2 cells, CT (ability to block fimbria) showed consistent results for mutant strain 119-14MT when used alone as starter (not combined with C. boidinii). Wild type (119WT) also showed a trend although of lower magnitude. ET (ability to block receptors in the cells) showed consistent reductions for wild type strain $119 \mathrm{WT}$ when used not combined. Regarding $C$. boidinii it was the only starter that significantly showed posi- 
tive results for AT, CT and ET when used alone. DT failed to demonstrated ability of any EPS samples to remove the pathogen once attached.

Conclusions: According to these results, L. pentosus 119WT and specially 119-14MT seem to be the most effective starters improving the antiadhesive properties of brine EPS, but not when combined with C. boidinii.

References: González-Ortiz et al.,2013. Vet Microbiology, 167(3-4), 494-499.

Salcedo et al., 2013. Food Chem, 136(2), 726-734.

Conflict of interest: Authors declare no conflict of interest.

\section{$309 / 46$ \\ SCREENING NOVEL FOODS FOR THE PREVEN- TION OF ETEC ATTACHMENT TO IPEC-J2 CELLS}

\author{
${ }^{(1)}$ Y. Zhu; (2) S. Martín Orúe. \\ (1) Sniba Department of Animal and Food Science University \\ Autonoma de Barcelona. Barcelona.; ${ }^{(2)}$ Animal Nutrition and \\ Welfare Service. Animal and Food Science Department. \\ Universitat Autònoma de Barcelona. Barcelona. Spain.
}

Introduction: Blocking or inhibiting enterotoxigenic E. coli (ETEC) fimbriae, or host receptors, by exogenous molecules of biologically active foods is considered a potential competition-based strategy to prevent and/or treat gastrointestinal disorders.

Objectives: The purpose of this study was to evaluate in vitro the potential of some novel foods to attach specifically enterotoxigenic E. coli (ETEC) K88 (adhesion test (AT)) and to reduce its attachment to intestinal porcine epithelial cells (IPEC-J2) (competition test (CT); exclusion test (ET); displacement test (DT)).

Methods: Tested ingredients included some cereals (highland barley, black rice, and little millet), two mushrooms (shiitake and jelly ear) and seaweed. All ingredients were finely ground, res-suspended in phosphate buffer saline (PBS) $(1: 10(\mathrm{w} / \mathrm{v}))$, vortexed and sonicated three times and then centrifuged to obtain the soluble extracts. Extracts were tested at 1 and $2 \%$.

Results: All cereal grains had the ability to adhere specifically ETEC K88 $(\mathrm{P}<0.01)$ and also shiitake and seaweed $(\mathrm{P}<0.05)$. No tested ingredient showed ability to attach non-fimbriated (NF) $E$. coli in a higher extent than PBS. In the CT (ability to block fimbria), highland barley, shiitake and seaweed reduced the attachment of ETEC K88 to IPEC-J2 cells $(\mathrm{P}<0.05)$. In ET (ability to block receptors) and DT (to remove attached bacteria), no one of the tested food extracts was able to reduce ETEC K88 adhesion compared to PBS.

Conclusions: Results from these experiments suggest that highland barley, black rice, little millet, shiitake and seaweed could offer receptor analogs to ETEC K88 fimbria, impairing in some cases the colonization of intestinal epithelial cells. These abilities could be behind part of the health benefits traditionally attributed to these ingredients. Further studies would be needed to ascertain the chemical structures involved.
References: González-Ortiz et al.,2013. Vet Microbiol, 167(34), 494-499. Salcedo et al., 2013. Food Chem, 136(2), 726-734.

Conflict of interest: Authors declare no conflict of interest.

\section{$309 / 47$ \\ USEFULNESS OF THE SIMGI ${ }^{\circledR}$ TO STUDY THE METABOLIC ACTIVITY OF PROBIOTICS. APPLI- CATION TO GRAPE POLYPHENOLS}

\author{
I. Gil Sánchez; C. Cueva; A. Tamargo; D. González de Llano; M. \\ Moreno Arribas; B. Bartolomé Suáldea.
}

Departamento de Biotecnología y Microbiología de Alimentos. Instituto de Investigación en Ciencias de la Alimentación (CIAL). CSIC-UAM. Madrid.

Introduction: Human gut microbiota influences the bioavailability of dietary polyphenols, as intestinal microbes metabolize them into active phenolic metabolites. On the other hand, certain probiotic strains (i.e. Lactobacillus plantarum CLC17) have been shown to metabolize polyphenols in culture studies ${ }^{1}$. Therefore, administration of these probiotics could be proposed as a nutritional strategy to improve the gut metabolism of polyphenols and enhance their health effects.

Objectives: To stabilize the probiotic strain L. plantarum CLC17 in the dynamic gastrointestinal simulator (simgi ${ }^{\oplus}$ ), and to study its impact in the microbial metabolism of a commercial polyphenol extract from red grapes.

Methods: Once faecal microbiota was inoculated and stabilized in the simgi ${ }^{\oplus}$, a first digestion of extract ( $800 \mathrm{mg}$, equivalent to $522 \mathrm{mg}$ of gallic acid) was carried out. After this, the system was washed, and then it was daily fed with L. plantarum CLC17 ( $6^{\star} 1010 \mathrm{CFU} /$ day). After 14 days, a second digestion of the grape extract $(800 \mathrm{mg}$ ) was carried out. This experimental sequence was repeated twice. Metabolic activity was monitored by determining microbial-derived phenolic metabolites and ammonium production. In addition, microbial diversity measures such as bacterial richness and diversity index were calculated.

Results: Feeding the simgi ${ }^{\oplus}$ with the extract activated the colonic metabolism leading to a battery of phenolic metabolites such as gallic acid, protocatechuic acid, 3-O-methylgallic acid, syringic acid, catechol and 3,4-dihydroxyphenylacetic acid, among others. In terms of microbial metabolism, the inclusion of L. plantarum caused a slight increase in the phenolic metabolism, especially in compounds of higher degree of polymerization, as well as, in the ammonium ion content. In addition, supplementation with $L$. plantarum CLC17 did not cause differences in bacterial diversity.

Conclusions: These results open the possibilities of using the probiotic strain L. plantarum CLC17 as a food ingredient to improve the metabolism of dietary polyphenols.

References: 1. González de Llano D et al. (2017) Eur Food ResTechnol 243: 531-538.

Conflict of interest: The authors declare that they have no conflict of interest.

9th Workshop SEPyP 


\section{$309 / 52$}

\section{RELEVANCE OF DIET AND SPECIFIC DIETARY COMPONENTS ON HUMAN GUT MICROBIOTA}

\author{
I. García Mantrana; M. Selma Royo; C. Alcántara Baena; M. Collado \\ Amores. \\ Institute of Agrochemistry and Food Technology (IATA) Spanish \\ Research Council (CSIC). Valencia. Spain.
}

Introduction: There is increasing evidence for the interaction between gut microbiome, diet and health ${ }^{1}$. Nowadays, developed societies are exposed to nutritional patterns characterized by high intakes of fats, proteins and processed foods that have been linked with higher risk of obesity, diabetes, cancer and cardiovascular disease $^{2}$. Despite the existing evidence of the effect of diet on gut microbiota composition, little is known about the impact of dietary patterns and nutrients on gut microbiota in healthy people.

Objectives: The aim of the study was to determine the effect of food consumption and adherence to Mediterranean diet on the gut microbiome of adults without pathology.

Methods: Dietary information $(n=27)$ was recorded using a full-length 140-item validated Food Frequency Questionnaire (FFQ). Adherence to the Mediterranean diet was measured using the PREDIMED test. Microbiota composition and diversity were obtained by 16S rRNA gene sequencing and specific quantitative polymerase chain reaction ( $\mathrm{qPCR}$ ). Microbial metabolic activity was determined by quantification of short chain fatty acids (SCFA) on high performance liquid chromatography.

Results: A higher Firmicutes-Bacteroidetes ratio was related to lower adherence to the Mediterranean diet, and greater presence of Bacteroidetes was associated with lower animal protein intake. High consumption of animal protein, saturated fats and sugars affected gut microbiota diversity. We also found that overweight individuals and lower MD adherence showed similar microbiota profile. Higher bifidobacterial counts, relative abundance of butyrate producing bacteria, and higher total SCFA were related to greater consumption of plant-based nutrients, such as vegetable proteins and polysaccharides. Better adherence to the Mediterranean diet was associated with significantly higher levels of total SCFA.

Conclusions: Diet and specific dietary components could affect microbiota composition, diversity and activity, which may have an effect on host metabolism by increasing the risk of Western diseases.

References: Graf et al., 2015. Myles, 2014

Conflict of interest: The authors declared no conflict of interest.

\section{9/54 \\ ROLE OF LACTOBACILLI BIOFILMS ON LISTERIA INNOCUA ADHESION}

\author{
(1) B. Orgaz Martín; (1)J. Jara Pérez; (1)L. Fernández Álvarez; (2)J. \\ Rodríguez Gómez. \\ (1) Department of Nutrition. Food Science and Food Technology. \\ Complutense University of Madrid. Madrid. Spain.; ${ }^{(2)}$ Professor. \\ Dpt. Nutrition. Food Science and Food Technology. Complutense \\ University of Madrid. Spain.
}

Introduction: Biofilms are microbial communities that grow attached to surfaces. In these communities, cells are embedded in an extracellular polymeric matrix (EPM) that functions as structural support, cellular defense mechanism and nutritional store for microorganisms. In general, biofilms are far more resistant than planktonic cells to the same treatments, hence their importance in clinical settings and food-related environments (Flemming et al. 2016). Many authors have proposed the use of probiotic microorganisms and/or their metabolites (e.g. nisin) as an anti-biofilm strategy. However, the ecological interactions in biofilms differ from those observed in free-floating cells, a fact often disregarded in such studies. Therefore, it is essential to test how certain probiotic strains behave when forming biofilms, and to know if their biofilms can modulate the adhesion of other microorganisms.

Objectives: The aims of this work were, first, to evaluate the ability of Lactobacillus fermentum ZL 26-21 and Lactobacillus salivarius $\mathrm{AP} 14 / \mathrm{Me} 2$ to form biofilms and, second, to evaluate if the presence of their preformed biofilms can modulate Listeria innocua adhesion.

Methods: Biofilms were developed in a batch system. Several parameters were measured over time: attached cell population, surface occupied by cells and total biomass (cells + EPM). Biofilms' 3D structure was visualized by confocal laser scanning microscopy.

Results: Both Lactobacillus strains were able to form robust biofilms after $24 \mathrm{~h}$. The presence of Lactobacillus preformed biofilms stimulated L. innocua adhesion. This suggests that whether the matrix itself or certain metabolites produced by lactobacilli and retained in this matrix, could promote L. innocua adhesion.

Conclusions: In conclusion, the use of probiotic microorganisms as an anti-biofilm strategy should be considered on a caseby-case basis, as it cannot be ruled out that certain strains may stimulate the adhesion of potentially pathogenic microorganisms.

References: Flemming, HC et al. Nature Reviews Microbiology (2016): 563-575

Conflict of interest: Authors declare no conflict of interest. 


\section{$309 / 56$}

VIABILITY AND STABILITY OF BIFIDOBACTERIUM ANIMALIS SSP. LACTIS AND LACTOBACILLUS ACIDOPHILUS IN SYNBIOTIC YOGURTS WITH OSLU

\author{
P. Delgado Fernández; O. Hernández-hernández; M. Villamiel \\ Guerra; A. Olano Villén; F. Moreno Andújar; N. Corzo Sánchez.
}

Department of bioactivity and food analysis.Research Institute of Food Science. CIAL (CSIC-UAM). Autonoma University of Madrid. Madrid. Spain.

Introduction: Pre- and probiotics have been widely used as functional ingredients due to their positive effects in the gastrointestinal tract and technological implications in the final product. Dairy industry is introducing new products based on these functional ingredients; however, there is a lack of knowledge in the effects of new generation prebiotics, as oligosaccharides derived from lactulose (OsLu), enzymatically produced by transgalactosylation, on the probiotics viability and stability. For an effective colonization, probiotic contents in yogurts should be more than $106 \mathrm{CFU} / \mathrm{g}$, but several factors in matrix such as acidity, $\mathrm{pH}$ or presence of prebiotics could affect their viability (1).

Objectives: Therefore, the aim of this work is to study the effect of two prebiotics, lactulose and OsLu, on viability of two probiotic strains, Bifidobacterium animalis ssp. lactis and Lactobacillus acidophilus, in symbiotic yogurts.

Methods: Yogurts were elaborated using S. thermophilus, $L$. bulgaricus and two probiotics, B. animalis ssp. lactis and L. acidophilus. Symbiotic yogurts were supplemented with lactulose and OsLu at 2 or $4 \%(\mathrm{w} / \mathrm{v})$. After fermentation, samples were stored for 4 weeks at $4^{\circ} \mathrm{C}$. Carbohydrates, $\mathrm{pH}$ evolution, short chain fatty acids (SCFAs) and lactic acid were determined during fermentation and storage. Moreover, viability of microorganisms was analyzed during storage.

Results: In yogurts with $4 \%$ of lactulose, the prebiotic was largely consumed (44\%) leading to an increase in lactic (1.1\%) and acetic $(0.4 \%)$ acids content, whereas OsLu was not metabolized by the starter regardless the assayed concentration. Probiotic strains viability increased in all symbiotic yogurts as compared to control, this effect was significantly higher for samples containing $4 \%$ prebiotic than those with $2 \%$.

Conclusions: In consequence, OsLu could represent a new alternative as a prebiotic supplement in yogurts due to its high stability with positive effects in maintaining probiotics strain stability during storage.

References: (1) Özer et al(2005).Rev.Agroquim.Tecnol.Aliment.11(1),19-24.

Conflict of interest: Authors declare no conflict of interest.

\section{$309 / 58$}

\section{GASTROINTESTINAL REDUCTION OF OCHRA- TOXIN A BY LACTIC ACID BACTERIA}

\author{
(1)J. Quiles Beses; ${ }^{(2)}$ R. Torrijos Caparrós; ${ }^{(3)} J$. Mañes Vinuesa. \\ (1)Investigador Predoctoral. Departamento de Medicina \\ Preventiva. Universitat de València. Valencia.; ${ }^{(2)}$ Estudiante de \\ Máster. Departamento de Medicina Preventiva. Universitat \\ de València. Valencia.; (3)Laboratory of Food Chemistry and \\ Toxicology. Faculty of Pharmacy. University of Valencia. Spain.
}

Introduction: Ochratoxin A (OTA) is a mycotoxin produced by the metabolism of fungus belonging to the genus Aspergillus and Penicillium. It is classified by the International Agency for Research on Cancer (IARC) as possibly carcinogenic to humans (group 2B) Human exposure to OTA occurs mostly by dietary intake, since it's a common contaminant in many foodstuffs such as cereals, wine, coffee, and in feeds for animals.

Objectives: Study the reduction of OTA during gastrointestinal digestion carried out with lactic acid bacteria (LAB).

Methods: Gastrointestinal digestion in vitro was carried out using 5L bioreactors Infors with a working volume of $4 \mathrm{~L}(2 \mathrm{G}$, $37^{\circ} \mathrm{C}$ ). Twenty grams of spiked loaf bread inoculated with OTA at a final concentration of $10 \mu \mathrm{g} / \mathrm{g}$ were mixed with $60 \mathrm{~mL}$ of artificial saliva. The bacterial strains were also added individually at $105 \mathrm{CFU} / \mathrm{mL}$. Gastric digestions ( $\mathrm{pH} 2,2 \mathrm{~h})$ and pancreatic $(\mathrm{pH}$ $6.5,2 \mathrm{~h}$ ) were simulated and aliquot of $20 \mathrm{~mL}$ of the gastric and duodenal fluid was sampled for the determination of the mycotoxins duodenal bioaccessibility. The OTA contained in gastric and duodenal fluids were analyzed by liquid-chromatographycoupled fluorescence detector (LC-FLD).

Results: The highest gastric bioaccessibility reduction was observed by the strain of $L b$. johnsonii CECT 289 with $98.1 \%$. At duodenal level the strain of $L b$. johnsonii CECT 289, confirmed the bioaccessibility OTA reduction with a data of $96.7 \%$ and showing a mean reduction around all the gastrointestinal digestion process of $97.4 \%$.

Conclusions: The strain of $L b$. johnsonii CECT 289 could be a very important candidate to be used as a starter culture for the bio control of the mycotoxin OTA during fermentative processes and could be important to reduce the intake of this toxic compound at intestinal level.

References: Manzini et al (2015). Food Control, 47. 203-211. Conflict of interest: Authors declare no conflicts of interest. 


\section{9/59}

\section{IMPACT OF MATERNAL BMI, PREGNANCY WEIGHT GAIN AND C-SECTION ON MATERNAL GUT AND MECONIUM MICROBIOTA PROFILE}

\author{
${ }^{(1)}$ M. Selma Royo; ${ }^{(1)}$ I. García Mantrana; ${ }^{(2)}$ A. Parra-Ilorca; ${ }^{(2)}$ M. Vento; \\ ${ }^{(1)} M$. Collado Amores. \\ (1)Institute of Agrochemistry and Food Technology (IATA) \\ Spanish Research Council (CSIC). Valencia. Spain.; (2)Division of \\ Neonatology. University and Polytechnic la Fe Hospital. Valencia. \\ Spain.
}

Introduction: Infant gut microbiota colonization process has been studied ${ }^{1}$, however the early in utero events and perinatal factors guiding the microbiome development have only recently become uncovered ${ }^{2}$. It has been suggested that shifts in maternal microbial signatures would be transferred to their offerings and they would play a role during fetal development and after birth.

Objectives: To characterize the impact of maternal BMI, weight gain and mode of delivery on maternal and meconium microbiome at birth.

Methods: Maternal stool $(\mathrm{n}=90)$ and meconium ( $\mathrm{n}=94)$ samples were collected at the birth. Microbiome composition was analysed by $16 \mathrm{~S}$ rRNA gene sequencing. Data related to maternal body mass index, weight gain during pregnancy, mode of delivery and neonatal parameters were collected.

Results: Mode of delivery had an important influence in the maternal gut $(\mathrm{p}=0.005)$ and meconium $(\mathrm{p}=0.001)$ microbiota. Peptoniphilus genus was enriched $(\mathrm{p}=0.001)$ in maternal gut from $\mathrm{C}$-section deliveries and it was correlated positively with higher maternal BMI $(\mathrm{p}=0.01)$.

Lower abundance of Bacteroides genus was found in meconium from $\mathrm{C}$-section compared to those observed in vaginal birth. Indeed, lower abundance of Bacteroides genus was associated with higher maternal pre-pregnancy BMI in both maternal gut $(\mathrm{p}=0.029)$ and meconium $(\mathrm{p}=0.033)$. Furthermore, weight gain during pregnancy had also an impact on maternal microbiome $(\mathrm{p}=0.046)$.

Differences in terms of maternal and meconium microbiota diversity related to mode of delivery or maternal BMI were no observed.

Conclusions: Maternal gut and meconium microbiota at birth are affected by pre-pregnancy BMI, weight gain and C-section. Our study suggests these perinatal factors are influencing maternal microbiota and that may have an influence in the microbial colonization of the newborn and in the immunological and metabolic programming with consequences on infant health at shortand long-term.

References: (1) Jakobsson et al.,2014.

(2) Mueller et al., 2016.

Conflict of interest: The authors declared no conflict of interest.

\section{$309 / 60$ \\ EVALUATION OF THE ANTIMICROBIAL ACTIVI- TY OF FERMENTED WHEY WITH KEFIR GRAINS BY RESPONSE SURFACE METHODOLOGY}

\author{
A. Monsalve Ríos; B. Salazar Alzate; M. Yepes Pérez. \\ Faculty of Science. National University of Colombia. Medellín. \\ Colombia.
}

Introduction: The discovery and application of antibiotics in human and animals ${ }^{1}$ has been one of the greatest technological advances of humanity; with this discovery, society thought that diseases caused by microorganisms would disappear, but soon was discovered that bacteria was able to generate antibiotics resistant mechanisms, causing a health global crisis ${ }^{23}$, that's why is necessary to search for new substances for the treatment of microbial infections. An interesting field of research is the antimicrobial activity of probiotics, specially in kefir grains which activity has been attributed to the production of organic acids, bacteriocins and kefiran, or by the competition against pathogens by adhesion to intestinal cells ${ }^{4}$.

Objectives: Give an added value to whey through its fermentation with kefir grains and, to test its antimicrobial activity against Staphylococcus aureus, Salmonella sp and Bacillus cereus.

Methods: The fermentation was carried out through response surface methodology with temperatures $\left(25^{\circ} \mathrm{C}-35^{\circ} \mathrm{C}\right)$, an initial concentration of grains $(2 \% \mathrm{p} / \mathrm{p}-10 \% \mathrm{p} / \mathrm{p})$, and a fermentation time (12-60 hour).

The antimicrobial activity was done using an agar diffusion method.

Results: Fermented whey with kefir grains has antimicrobial activity against the bacteria tested, presenting inhibition zones between $8 \mathrm{~mm}$ and $15 \mathrm{~mm}$.

Conclusions: Fermented whey with kefir grains could be a promising source of compounds with antimicrobial activity.

References: [1] M. Teuber, "Spread of antibiotic resistance with food-borne pathogens," Cell. Mol. Life Sci., vol. 56, no. 9-10, pp. 755-763, Dec. 1999.

[2] A. Quizhpe Peralta, L. Encalada Torres, and A. Sacoto Molina, "Uso Apropiado de Antibioticos y Resistencia Bacteriana," Afeme, p. 168, 2014.

[3] J. Madigan, M.T., Martinko, J.M., Parker, Biología de los microorganismos. 2006.

[4] Z. B. Guzel-Seydim, T. Kok-Tas, A. K. Greene, and A. C. Seydim, "Review: functional properties of kefir.," Crit. Rev. Food Sci. Nutr., vol. 51, no. 3, pp. 261-8, 2011.

Conflict of interest: There is no conflict of interest related to this text 


\section{9/67}

\section{ANTIMICROBIAL ACTIVITY OF SPORULATED BA- CILLUS ISOLATED FROM THE INTESTINE OF CA- CHAMA BLANCA (PIARACTUS BRACHYPOMUS)}

\author{
${ }^{(1)}$ D. Moreno Blandon; ${ }^{(2)}$ O. Montoya Campuzano; ${ }^{(3)}$ L. Puello \\ Caballero. \\ ${ }^{(1)}$ Faculty of Health Sciences. Colegio Mayor of Antioquia. \\ Medellín-Antioquia. Colombia.; (2)Sciences Faculty. National \\ University of Colombia. Medellín headquarters. Medellín- \\ Antioquia. Colombia.; ${ }^{(3)}$ Faculty of Agrarian Sciences. National \\ University of Colombia. Medellín headquarters. Medellín- \\ Antioquia. Colombia.
}

Introduction: Some sporulated Bacillus are considered probiotics and are administered in animal feed to avoid the use of antibiotics as growth promoters (APC), among them are Bacillus pumilus, Bacillus megaterium, among others.

Objectives: Evaluate the antibacterial activity of some species of sporulated Bacillus isolated from the intestine of Cachama Blanca.

Methods: The strains were previously isolated and provided by the Laboratory of Water and Food Microbiology of the National University of Colombia, Medellín. They were recovered in the BHI enrichment broth and subsequently inoculated in the culture médium Plate Count, from which the isolates subjected to the activity tests, previously identified by the API 50CHB system, were obtained. The activity was evaluated using: diffusion in box (Kirby Bauer), microdilution in broth (Multiskan Go) and turbidimetry by spectrophotometer.

Results: Bacillus pumilus showed bactericidal activity against Aeromonas hidrophyla and Pseudomonas sp, and like Bacillus licheniformis, exerted bacteriostatic action against Staphylococcus aureus, Pseudomonas aeruginosa and Salmonella sp.

Conclusions: The two species of Bacillus, showed ability to inhibit pathogens as A. hidrophyla and Pseudomonas sp and delay the growth of $S$. aureus, Pseudomonas aeruginosa and Salmonella $s p$. Bacillus sp. It presumes benefits for the treatment of gastrointestinal diseases, because they generate widely favorable metabolic compounds.

References: Simon M. Cutting. (2011) Bacillus probiotics. Review. 214e220. doi.org/10.1016/j.fm.2010.03.007. 2.SangkiLee, JoongiaeLee, Yong-IkJin, Jin-Cheo lJeong, Yoon HyukChang, YoungseungLee, YoonhwaJeong, MisookKim. Probiotic characteristics of Bacillus strains isolated from Korean traditional soy sauce. (2017). doi.org/10.1016/j.lwt.2016.08.040.3. Parker EA, Roy T, D'Adamo CR, Wieland LS, Probiotics and Gastrointestinal Conditions: An Overview of Evidence from the Cochrane Collaboration, Nutrition (2017). doi: 10.1016/ j.nut.2017.06.024.

Conflict of interest: The authors do not declare having conflicts of interest.

\section{9/76. \\ ADHESIVE ABILITY AND INHIBITION OF PATHO- GEN ADHESION TO HUMAN INTESTINAL CELLS OF BIFIDOBACTERIUM STRAINS}

\author{
${ }^{(1)}$ K. Busca Arenzana; ${ }^{(2)}$ K. Murphy; ${ }^{(3)}$ R. Ross; ${ }^{(4)}$ C. Stanton. \\ (1)Teagasc Food Research Centre. Moorepark. Cork. Ireland.; ${ }^{(2)} \mathrm{Apc}$ \\ Microbiome Institute. University College. Teagasc Food Research \\ Centre.Moorepark. Fermoy. Cork. Ireland.; ${ }^{(3)}$ Apc Microbiome \\ Institute. University College. Teagasc Food Research Centre. \\ School of Science, Engineering \& Food Science. University \\ College. Moorepark. Fermoy. Cork. Ireland.; ${ }^{(4)}$ Apc Microbiome \\ Institute. Teagasc Food Research Centre.Moorepark. School of \\ Microbiology. University College. Cork. Ireland.
}

Introduction: Intestinal colonisation by commensals bacteria, in particular bifidobacteria (frequently present in high numbers in the human gut) is associated with several benefits for the host, including inhibition of colonisation by pathogens.

Objectives: Bifidobacterium species are widely used as probiotics and are highly competitive in the complex intestinal community. The objective here was to study the adhesive ability of three Bifidobacterium strains isolated from healthy infants and the inhibition of pathogens using in vitro tissue culture techniques.

Methods: Three Bifidobacterium species: B. breve, B. bifidum and $B$. longum previously isolated from infant stool, were utilized (individually and in combination) for adhesion and competitive exclusion assays. Adhesion capability was assessed using the epithelial intestinal cell line HT29 derived from human colon carcinoma. Salmonella enterica was chosen for the competitive exclusion assays due to their role as a human pathogen. The widely used probiotic Bifidobacterium animalis subsp. lactis Bb12 was included as a positive control.

Results: Adhesion (expressed as the percentage of bacteria adhered with respect to the amount of bacteria added) ranged between 0.2 and $0.8 \%$ depending on the Bifidobacterium strain. All the bifidobacterial strains decreased the adhesion of S. enterica (between 40-60\% adhesion inhibition).

Conclusions: These preliminary results are in concordance with previous reports and will be included as part of $B$. breve, $B$. bifidum and $B$. longum phenotypical characterization according to international guidelines for probiotics.

References: Arboleya, S., Ruas-Madiedo, P., Margolles, A., Solís, G., Salminen, S., de Los Reyes-Gavilán CG., Gueimonde, M. 2011. Characterization and in vitro properties of potentially probiotic Bifidobacterium strains isolated from breast-milk. Int J Food Microbiol.149 (1):28-36.

Conflict of interest: All authors declare that no competing interest exits. 


\section{9/77. \\ PROBIOTIC BACTERIA IN HUMAN MILK AFTER SPRY-DRIED}

\author{
${ }^{(1)}$ A. Rodriguez Arreola; (2)J. Solis Pacheco; ${ }^{(3)}$ A. Cabazos Garduño; \\ (4) J. Gutierrez Padilla; ${ }^{(5)}$ J. Serrano Niño; ${ }^{(6)}$ E. Garcia Morales; ${ }^{(7)} O$. \\ Gonzalez Reynoso; ${ }^{(2)}$ B. Aguilar Uscanga.
}

\begin{abstract}
(1) PhD in Biotechnological Processes. Chemical Engineering Department. University of Guadalajara. Guadalajara. Mexico.; (2) Pharmacobiology Department. University of Guadalajara. Guadalajara. Mexico.; ${ }^{(3)}$ Departamento de Farmacobiologia. Universidad de Guadalajara. Guadalajara. Mexico.; (4)Pharmacobiology Department. University of Guadalajara. Mexico.; ${ }^{(5)}$ Pharmacobiology Department. University of Guadalajara. Guadalajara. Mexico.; ${ }^{(6)}$ Neonatology. Civil Hospital of Guadalajara. Guadalajara. Mexico.; ${ }^{(7)}$ Chemical Engineering Department. University of Guadalajara. Guadalajara. Mexico.
\end{abstract}

Introduction: Human milk is the ideal food to feed children during their first six months of life1, besides is a key factor in the initiation and development of the intestinal microbiota of neonates because it ensures a continuous supply of bacteria throughout the lactation2.However, traditional methods for its conservation could affect the nutritionals and microbiological properties.

Objectives: Analyze the content of lactic bacteria in samples of human milk subjected to a spray drying process to verify the viability of them as well as the possible use of them as potential probiotics.

Methods: Microbiological analyses were performed on samples of human milk before and after the spray drying process and it was added in MRS agar at $37^{\circ} \mathrm{C}$ for $48 \mathrm{~h}$. The CFU obtained were isolated and identified by miniaturized system API 50 CHL. On the other hand, a group of mice $\mathrm{CEBAB} / \mathrm{C}$, was fed during eleven weeks with reconstituted powder human milk, and they were compared with a group of mice fed with commercial formula milk and a control group without feeding them with milk. The feces of each group were collected per week and the presence of lactic bacteria analyzed.

Results: In the group fed with the spray-dried milk the following microorganism were found Lactobacillus fermentum, Lactobacillus raffinolactis, Lactobacillus paracasei and Lactobacillus crispatus; the group fed with commercial milk formula were found Lactobacillus reuteri and Lactobacillus salivarius; the group fed without milk Lactobacillus salivarius was found.

Conclusions: After spray-dried milk treatment, the probiotic bacteria still are able to proliferate in the intestine of mice.

References: 1 . Aguilar Palafox M.A., Fernández Ortega M.A. (2007).Lactancia materna exclusiva. Rev. Fac. Med. 50:174-179. 2. García L.R.(2011).Composición e inmunología de la leche humana. Ac. Pediatr.Mex. 32:223-230.

Conflict of interest: I declare that the information provided is true and that I am not aware of any other circumstance that constitutes a conflict of interest, whether effective, potential or apparent.

9th Workshop SEPyP

\section{$309 / 79$.}

\section{VITAMIN B2 OVER-PRODUCING LAB AND THEIR APPLICATION IN BIO-FORTIFIED CERE- AL-BASED FUNCTIONAL FOOD}

\begin{abstract}
${ }^{(1)} A$. Yépez Latorre; ${ }^{(2)}$ S. Hernández Recio; ${ }^{(3)} P$. Russo; ${ }^{(3)} V$. Capozzi; ${ }^{(2)} M$. Mohedano; ${ }^{(4)} G$. Spano; ${ }^{(2)} P$. López; ${ }^{(5)} R$. Aznar Novella.

${ }^{(1)}$ Department of Microbiology and Ecology. University of Valencia (UVEG). Burjassot. Valencia. Spain.; ${ }^{(2)}$ Department of Molecular Microbiology and Infection Biology. Biological Research Center (CIB-CSIC) Madrid. Spain.; ${ }^{(3)}$ Department of the Science of Agriculture. Food and Environment. University of Foggia. Foggia. Italy.; ${ }^{(4)}$ Department of the science of agriculture, food and environment, university of foggia; ${ }^{(5)}$ Department of Microbiology and Ecology. University of Valencia (UVEG). Burjassot. Institute of Agrochemistry and Food Technology (IATA-CSIC). Spanish Type Culture Collection. University of Valencia. Paterna. Valencia. Spain.
\end{abstract}

Introduction: Riboflavin intake is normally supplied by a balanced diet; however, the deficiency still occurs in both developing and industrialized countries. Lactic acid bacteria (LAB) are able to produce this group of vitamins, thus they can be used to naturally increase riboflavin concentrations in new fermented and functional food.

Objectives: To select riboflavin over-producing Lactobacillus plantarum mutants from a collection of riboflavin producer LAB strains.

To set up a direct fluorescence spectroscopy detection method to quantify riboflavin as a rapid test for strains selection of B2.

To test the selected riboflavin-producing LAB strain to improve the nutritional value of cereal-based kefir-like products

Methods: Five riboflavin producers $L$. plantarum strains were exposed to increasing concentrations of roseoflavin (Burgess et al., 2006).

Riboflavin production by the strains was determined in culture supernatants by HPLC-fluorescence method (Russo et al., 2014) and by direct fluorescence spectroscopy.

Fermentation of cereal infusions was carried out using commercial kefir starters together with selected riboflavin over-producing L. plantarum strain.

Results: Riboflavin fluorescence quantification in culture supernatants with and without HPLC fractionation revealed similar values and showed an increase between 2- and 27-fold compared to the wild-type strains production. Among the overproducing strains, L. plantarum M5MA1-B2 -secreted the highest riboflavin levels (around $3 \mathrm{mg} \mathrm{L}-1$ ).

Riboflavin quantification of kefir-like beverages produced with L. plantarum M5MA1-B2 revealed $1.5 \mathrm{mg} \mathrm{L-1}$ vitamin B2 content in oat kefir-like, representing the highest production level (about 2.5-fold higher than non-fermented matrix).

Conclusions: A set of L. plantarum strains able to produce riboflavin up to $3 \mathrm{mg} \mathrm{L}-1$ were obtained and M5MA1-B2 showed to be proficient as riboflavin producer in a cereal-based fermented matrix when co-inoculated with starter cultures.

Fluorescence analysis of culture supernatants permitted to standardize an efficient method for vitamin B2 quantification which is suitable for rapid determination assays in further studies. 
References: Burgess et al., 2006. https://doi.org/10.1186/14752859-5-24

Russo et al., 2014. https://doi.org/10.1007/s00253-013-5484-7

Conflict of interest: The authors declare the absence of any conflict of interest.

\section{$309 / 80$ \\ ATTACHMENT SPECIFIC GENE EXPRESSION CHANGES PROMOTED BY L. SALIVARIUS LV72 AND HELA-CELLS COEXISTENCE. PHENOTYPIC CONSEQUENCES}

\section{Martín Cueto; L. Quirós Fernández; J. Suárez Fernández.}

Area of Microbiology. Department of Functional Biology. Faculty of Medicine. University of Oviedo. Spain.

Introduction: The adherence of the microbiota to the mucosal surfaces is mediated by the bacterial adhesins and the epithelial receptors. OppA plays a prominent role as adhesin for lactobacilli, the mucosal receptors being the glycocalix proteoglycans.

Objectives: The expression changes of the L. salivarius Lv72 oppA determinant and those of $62 \mathrm{HeLa}$-cell genes involved on proteoglycan biosynthesis, upon interaction of both cell types, are presented.

Methods: qPCR and inmunological binding were used for transcription and protein detection, respectively.

Results: The transcription of $o p p A$ increased 100 fold in the $30 \mathrm{~min}$ that followed culture mixing and remained high for 24 h. High increases of OppA were observed on the L. salivarius surface. Conversely, the expression of about $1 / 3$ of the genes coding for proteoglycans protein moieties and for polymerization/modification of their attached glycosaminoglycans, diminished, while no changes were observed for the rest. This corresponded with a minor concentration of proteoglycans on the HeLa cells surface, although with increased lengths of their polysaccharides. The attachment between the bacteria and the epithelial cells gradually increased during the first $24 \mathrm{~h}$ of mixed incubation.

Conclusions: Lactobacilli alternate a free-living with a mutualistic, mucosa-attached existence, where adhesins become useful. It is thus logical that expression of $o p p A$ is enhanced upon contact with HeLa cells. These, on the contrary, are always covered by lactobacilli, thus implying that pure epithelial cultures are "unnatural". Therefore, enhanced expression of the surface proteoglycan genes would be expected to maximize the possibility of lactobacilli binding to their surface, which would be followed by return to the basal expression levels, when the protection conferred by the resident lactobacilli, would be restored.

References: Martin R et al. (2013). Surface glycosaminoglycans mediate adherence between HeLa cells and Lactobacillus salivarius Lv72. BMC Microbiol.

Conflict of interest: The authors declare the absence of any conflict of interest.

\section{9/84. \\ EQUIVALENCE OF A NOVEL L.RHAMNOSUS ISO- LATE TO THE REFERENCE ATCC53103 STRAIN}

\author{
(1) J. Espadaler Mazo; ${ }^{(2)}$ E. Astó Sanchez-Lafuente; ${ }^{(2)}$ I. Mendez \\ Sobrado; ${ }^{(2)}$ E. Navarro Tapia. \\ ${ }^{(1)}$ AB-Biotics S.A. Barcelona. Spain.; ${ }^{(2)}$ Innovation department. AB- \\ Biotics S.A. Barcelona. Spain.
}

Introduction: Lactobacillus rhamnosus GG (ATCC53103) is the bacterial strain with more grade- 1 evidence-based clinical indications ${ }^{1}$. This strain was patented in $1985^{2}$ and thus now can be available as a generic.

Objectives: To assess the equivalence of a L. rhamnosus isolate from AB-Biotics SA (Barcelona, Spain) to the original L. rhamnosus ATCC53103.

Methods: DNA of the new isolate was sequenced using MiSeq. Data was assessed and compared to the published reference genome of L.rhamnosus ATCC53103 (GenBank accession number NC_013198.1) using the programs prinseq-lite, bwa_mem, freebayes and the R package [3]. Survival of the new isolate and of Lactobacillus rhamnosus ATCC53103 was measured after $3 \mathrm{~h}$ of incubation in synthetic postprandial gastric juice ( $\mathrm{pH} 4$ ), MRS broth at various $\mathrm{pH}$ and MRS broth $+0.5 \%$ bile. Basic enzymatic activities were determined using API 50CHL and API ZYM20 (BioMerieux).

Results: The genome of the new L. rhamnosus isolate is identical to the reference genome of L. rhamnosus ATCC53103 at $>99.999 \%$. The difference was due to 12 SNPs and short inframe deletions, none of them in the pilin spaCBA cluster. In comparison, Sybesma et al. found 19 SNPs and short indels when comparing a L. rhamnosus ATCC53103 from an official collection to the published reference genome [4]. Moreover, survival to simulated gastrointestinal conditions and API enzymatic profiles produced identical results for the original Lactobacillus rhamnosus ATCC53103 and the new isolate.

Conclusions: This new L. rhamnosus isolate appears to be equivalent to the original Lactobacillus rhamnosus ATCC53103 strain.

\section{References:}

[1] Guarner, F., et al. World Gastroenterology Organization Global Guidelines. Probiotics and Prebiotics. 2017

[2] Gorbach, SL., Goldin, BR., Lactobacillus strains and methods of selection. US 4839281. 1985

[3] The R Core Team. The R Project for Statistical Computing. 2012. http://www.r-project.org/

[4] Sybesma, W. et al. Appl Environ Microbiol. 2013. 79(7):22332239

Conflict of interest: Authors are full-time employees of AB-BIOTICS SA. 9th Workshop SEPyP 


\section{$309 / 86$.}

\section{DEVELOPMENT OF PROBIOTIC SYRUPS}

${ }^{(1)}$ S. Cruz Sousa; (1)O. Martins; ${ }^{(1)}$ A. Freitas; ${ }^{(2)}$ A. Carvalho; ${ }^{(1)} A$. Gomes.

${ }^{(1)}$ Catholic University of Portugal. CBQF. Centre of Biotechnology and Fine Chemistry. Associate Laboratory. College of Biotechnology. Porto. Portugal.; ${ }^{(2)}$ REQUIMTE/LAQV - ISEP, Engineering Institute of the Polytechnical Institute of the University of Porto. Porto. Portugal.

Introduction: Probiotics have the capability of providing beneficial effects to human health and are used to recover symbiosis between colonic microbiota and the host or reestablish states of dysbiosis. In order to achieve such role a minimum of $10 \mathrm{E} 7$ viable cells per gram of product, at the time of consumption, is required, as established by the International International Scientific Association for Probiotics and Prebiotics ${ }^{1}$. As such, not every product can be utilized to incorporate and deliver probiotics to different target groups.

Objectives: This study aimed at developing syrups able to sustain probiotic viability, that could be used as probiotic delivery vectors for both children and the elderly (with difficulties in swallowing), and testing their stability.

Methods: To three types of syrups, namely, standard syrup, cocoa syrup and syrup for diabetics, a suspension of probiotic was added at $10 \%(\mathrm{v} / \mathrm{v})$ inoculum. Five strains of probiotics were tested. Viability of the probiotics and free sugars were assessed throughout the 3 month storage period.

Results: Standard syrup presented high amount of free sugars and was not able to sustain the necessary probiotic viability even for one week. Cocoa syrup showed lower initial levels of free sugars and was able to maintain viability above the established limit for 30 days for L. casei $431^{\circ}$, for 45 days for B. animalis Bo, and for 60 days for $L$. casei ssp. paracasei LAFTI $^{\circledR}$ L26. Syrup for diabetics presented very low free sugars and also good viability throughout storage, with L. casei-01 lasting for 30 days and B. animalis Bo, and L. casei ssp. paracase $\mathrm{LAFTI}^{\circledast} \mathrm{L} 26$, for 60 days.

Conclusions: Results showed the possibility of developing probiotic syrups, by maintaining cell viability within the required values.

References: 1. Hill, Colin. et al. (2014). Nature Reviews Gastroenterology \& Hepatology, 11, 506-514.

Conflict of interest: Authors declare no conflict of interest.

9th Workshop SEPyP

\section{Track 4: Veterinary}

\author{
Reviewers: \\ Teresa Requena Rolanía \\ Research Institute in Food Sciences, CIAL (CSIC- UAM). Madrid. \\ Juan Evaristo Suárez Fernández \\ Oviedo University. Asturias. \\ Juan Miguel Rodríguez Gómez \\ Veterinary School. Universidad Complutense de Madrid.
}

Abelardo Margolles Barro

Institute of Dairy Products of Asturias, IPLA-CSIC. Oviedo.

\section{9/16 \\ THE ASTURCON HORSE: A MODEL FOR HORSE MICROBIOME STUDIES AND SOURCE OF PROBI- OTICS}

${ }^{(1)} M$. Gueimonde; ${ }^{(2)} D$. Ríos-covían; ${ }^{(3)} L$. Ang; ${ }^{(4)} C$. Gómez-Gallego; ${ }^{(5)} A$. García Pérez; ${ }^{(1)} C$. De Los Reyes-Gavilán; ${ }^{(4)}$ S. Salminen.

\begin{abstract}
(1)Department of Microbiology and Biochemistry of Dairy Products. Instituto de Productos Lácteos de Asturias (IPLA-CSIC). Group Diet, Microbiota and Health. Instituto de Investigación Sanitaria del Principado de Asturias (ISPA). Oviedo. Spain.; (2)Department of Microbiology and Biochemistry of Dairy Products. Dairy Research Institute of Asturias (IPLA-CSIC). Villaviciosa. Asturias. Spain.; ${ }^{(3)}$ Precision medicien centrer. ${ }^{(1)}$ st affiliated Hospital of Zhengzhou University. Zhengzhou. China.; ${ }^{(4)}$ Functional foods forum. Faculty of medicine. University of Turku. Turku. Finland.; ${ }^{(5)}$ Asociación Pura Raza Asturcón. Oviedo. Asturias. Spain.
\end{abstract}

Introduction: The Asturcon (Asturias) is among the oldest horse-breeds, with written references dating back to $80 \mathrm{BC}$. Crossbreeding threatened the breed until the last century 70's, with less than 50 in-breed wild-animals left, when measures were implemented for its protection. Since then, any Asturcon is genotyped to ensure in-breeding and registered in the Genealogical Book.

Domestic horses are more sensitive to gastrointestinal diseases than wild/feral animals, this being also observed in the Asturcon. Thus we suggest that the ancient microbiota reflected in wild-Asturcons may be protective and that changes induced during domestication may have reduced microbiota resilience and resistance to diseases. At present, the co-existence of wild, ranched and tamed/ stabled Asturcons provides a useful model to characterize the impact of domestication on the microbiota. The wild-animals may also represent the original ancient microbiota for the isolation of specific strains or combinations aimed at enhancing animal health.

Objectives: Our objective was to compare the microbiomes of wild, ranched and stabled Asturcons. We also aimed at characterizing the Lactobacillus population and isolating strains as potential horse probiotics.

Methods: Fresh fecal samples from nine Asturcon mares (three wild, three ranched and three tamed-stabled) were placed 
in anaerobiosis jars and transported/processed within 3 hours from collection. Samples were homogenized, serially diluted and plated in MRS for isolation of lactobacilli. $50 \mathrm{~mL}$ of homogenate were centrifuged, the pellets used for microbiome analyses and the supernatants for SCFA measurement.

Results: Wild-horses' showed microbiota differences when compared with domesticated animals, presenting also higher levels of fecal SCFA. All the Lactobacillus strains isolated belonged to the species Lactobacillus equi, Lactobacillus equigenerosi or Lactobacillus hayakitensis.

Conclusions: Our results suggest an impact of domestication on horse microbiome. The isolates of L. equi, L. equigenerosi and L. hayakitensis obtained here constitute a potential source for the development of probiotics for horses.

Conflict of interest: Authors declare no conflict of interest.

\section{$309 / 22$ \\ B-GLUCAN-PRODUCING PEDIOCOCCUS PARVU- LUS 2.6: CHARACTERIZATION OF ITS PROBIOTIC AND IMMUNOMODULATORY PROPERTIES}

\author{
${ }^{(1)}$ A. Pérez-Ramos; ${ }^{(1)}$ M. Mohedano; (2)M. Pardo; (1)P. López. \\ ${ }^{(1)}$ Molecular Microbiology and Infection Biology. Biological \\ Research Center. Madrid. Spain.; ${ }^{(2)}$ Food Research Division. Azti- \\ Tecnalia. Derio. Spain.
}

Introduction: Pediococcus parvulus 2.6 isolated from cider secretes a 2 -substituted $(1,3)$ - $\beta$-D-glucan ( $\beta$-glucan). Previous in vitro studies supported the potential of $P$. parvulus 2.6 as a probiotic strain and of its $\beta$-glucan as an immunomodulator and prebiotic compound (1). Currently, Danio rerio (zebrafish) is a useful model to perform in vivo interactomic studies of probiotic bacteria in the digestive tract. Moreover, fluorescent labelling could be a powerful tool for evaluation of bacterial probiotic properties in zebrafish models.

Objectives: Thus, the aim of this work was to evaluate $P$. parvulus 2.6 and its $\beta$-glucan, using zebrafish models.

Methods: $P$. parvulus 2.6 and the EPS-non-producing $2.6 \mathrm{NR}$ isogenic strain were fluorescently tagged with mCherry and used for interactomic studies. A gnotobiotic embryonic zebrafish model was utilized for bacterial colonization and competition with a pathogen, as well as to evaluate the immunomodulation capability of the $\beta$-glucan. Besides, a transgenic (mpx:GFP) zebrafish model was used to determine the influence of the polysaccharide in an induced inflammation model.

Results: The results showed a positive effect of $\beta$-glucan in zebrafish gut colonization by $P$. parvulus. The same positive effect of the polymer was detected in vitro in interactomic studies with human Caco-2 cells, since 2.6NR strain supplemented with purified $\beta$-glucan almost recovered the adhesion levels of 2.6 strain. Furthermore, $P$. parvulus was able to compete with Vibrio anguillarum (more efficiently in the presence of $\beta$-glucan), protecting zebrafish against infection. Expression levels of several immune response genes were evaluated, and the results showed a decrease on the expression of pro-inflammatory cytokine genes. Finally, the use of an induced inflammation model revealed that $\beta$-glucan seems to have an anti-inflammatory effect.

Conclusions: To conclude, we have demonstrated a positive influence of the $\beta$-glucan in bacterial adhesion and in the prevention of pathogen infection, as well as its potential as immunomodulator.

References: Pérez-Ramos 2015 ISBN: 9780128021897

Conflict of interest: Authors declare no conflict of interest.

\section{9/37. \\ ISOLATION OF LACTIC ACID BACTERIA FROM THE COCK FEATHER DE LEÓN AND FROM PAR- TRIDGE}

\author{
(1) M. Garcia Diez; (1)L. Alvarez Gonzalez; ${ }^{(2)}$ J. Marca Puig; ${ }^{(3)}$ R. Miranda \\ Hevia; ${ }^{(3)}$ M. Gomez Garcia; ${ }^{(4)}$ S. Costillas Alonso; ${ }^{(3)}$ A. Carvajal \\ Urueña; ${ }^{(3)}$ P. Rubio Nistal. \\ ${ }^{(1)}$ Aquilón CyL. León. Spain.; ${ }^{(2)}$ Aquilón CyL. León. Spain.; ${ }^{(3)}$ Group \\ Digesporc. Department Animal Health. Faculty of Veterinary. \\ University of León. León. Spain.; ${ }^{(4)}$ Departamento de Sanidad \\ Animal. Facultad de Veterinaria. León. Spain.
}

Introduction: The extensive breeding system of the cock feather de León, recognized for the brightness of its feathers used to make fishing flies, together with the wild habitat of the partridge (Perdix perdix), make these two breeds of birds an excellent source of bacteria with probiotic potential that could encourage an adequate intestinal microbiota in different stages of bird growth and decrease the incidence of different pathological processes as well as the use of antibiotics.

Objectives: Our objective was to characterize lactic acid bacteria from samples of cock feather and partridge that could have probiotic properties.

Methods: A total of 120 isolates identified as Lactobacillus spp. were recovered from 10 samples of cock feather and 3 samples of partridge. In a first step, isolates sensitive to the antibiotics recommended by EFSA were selected. Secondly, resistance to acid $\mathrm{pH}$ and bile salts were checked as well as acidification capacity. Finally, selected isolates were confronted with 5 pathogenic microorganisms.

Results: 39 out of the 120 isolates met the EFSA requirements regarding antibiotic resistance. Taken into account their resistance to acid $\mathrm{pH} /$ bile salts and acidification capacity, a total of 17 isolates were finally selected and confronted with five pathogens, evaluating the halo of inhibition of the growth.

Conclusions: Five isolates of lactic acid bacteria with probiotic potential were finally selected. They are being used in experimental tests to check their safety and efficacy, selecting the combination of strains with the best results.

References: Estrada, O. 2015. Tesis doctoral. Departamento Sanidad Animal. Universidad de León.

Kabir, L. 2009. International Journal of Molecular Science 10, 3531-3546.

Conflict of interest: The authors of this paper declare that they are not in a situation of conflict of interest with respect to the present study. 


\section{9/43}

SERUM BIOMARKERS TO DETERMINE INTESTINAL BARRIER INTEGRITY IN PIGLET MODELS

\author{
(1) P. López Colom; ${ }^{(2)}$ K. Yu; ; ${ }^{(3)}$ E. Barba Vidal; ${ }^{(1)}$ S. Martín Orúe; ${ }^{(3)}$ L. \\ Castillejos; ${ }^{(3)} D$. Solà Oriol; ${ }^{(2)} A$. Bassols. \\ (1)Animal Nutrition and Welfare Service. Animal and Food Science \\ Department. Universitat Autònoma de Barcelona. Barcelona. \\ Spain.; ${ }^{(2)}$ Departament de Bioquímica i Biologia Molecular. \\ Universitat Autònoma de Barcelona. Barcelona.; (3)Servicio de \\ Nutrición y Bienestar Animal. Departament de Ciència Animal i \\ dels Aliments. Universitat Autònoma de Barcelona. Barcelona.
}

Introduction: This work proposes safe and non-invasive biomarkers for monitoring gut wall integrity in gastrointestinal disorders as a possible tool to evaluate probiotic therapies. We explore its usefulness in piglets as a suitable animal model to assess microbiota-health interactions.

Objectives: To determine three serum biomarkers: intestinal fatty acid-binding protein (I-FABP, a marker of intestinal cell damage); tumour necrosis factor (TNFa, a pro-inflammatory cytokine) and pig-major acute-phase protein (PigMAP, an acute-phase protein) and their correlation to histomorphological intestinal parameters in weaning pigs with a broad range of gut damage.

Methods: One week after weaning, a total of 80 weaned piglets from different clinical trials were subjected to an oral challenge with Salmonella Typhimurium or enterotoxigenic (ETEC) Escherichia coli K88. Animals were euthanized 4 or 8 days post-inoculation (PI) and blood samples and ileal tissue collected. I-FABP, TNFa and PigMAP were analysed by different ELISA adapted methods. Histomorphometrical measurements included: villus height $(\mathrm{VH})$, crypt depth (CD), counts of intraepithelial lymphocytes (IEL), goblet cells (GC) or mitosis (MIT). Spearman's correlations $(\rho S)$ were calculated among all serum markers and histological values.

Results: Serum biomarkers were intercorrelated $(\mathrm{P}<0.01)$ (I-FABP-TNF $\alpha \rho=0.376$; I-FABP-PigMAP $\rho S=0.326$, and $\mathrm{TN}$ $\mathrm{F} \alpha$-PigMAP $\rho S=0.392$ ). The three biomarkers correlated with all histological parameters except GC with TNFa and PigMAP and MIT with PigMAP $(\mathrm{P}>0.05)$. VH showed higher correlations with TNF $\alpha$ and PigMAP compared to I-FABP $(\rho S=-0.535, \rho S=0.588$ and $\rho S=0.391$ respectively). $C D$ and MIT were highly correlated to I-FABP $(\mathrm{CD} \rho \mathrm{S}=0.527, \mathrm{MIT} \rho \mathrm{S}=0.363)$ when compared to $\mathrm{TNFa}$ (CD $\rho S=0.318$, MIT $\rho S=0.277)$ or PigMAP (CD $\rho S=0.261$, MIT $\rho S=0.096)$.

Conclusions: Serum I-FABP, TNFa or PigMAP were sensitive to tissue injury and significantly correlated with histological variables studied. Interestingly, underlying physiological mechanisms (local or systemic response) might cause the different correlation strengths.

References: Bischoff et al. 2014. BMC Gastroenterol. 14:189. Piñeiro et al. 2009. Vet J. 179(1):78-84.

Conflict of interest: Authors declare no conflict of interest.

\section{$309 / 48$ \\ PROBIOTIC CHARACTERIZATION OF LACTIC ACID BACTERIA ISOLATED FROM MARINE FISH GUTS}

\author{
${ }^{(1)}$ A. Hernández De Rojas; ${ }^{(1)}$ S. Alonso Fernández; ${ }^{(1)} C$. Castro Pérez; \\ ${ }^{(2)}$ X. Moreno Ventas-Bravo; ${ }^{(3)}$ I. García De La Banda. \\ (1)Spanish Institute of Oceanography. Oceanographic Center of \\ Gijón. Gijón. Spain.; ${ }^{(2)}$ School of Civil Engineering. University of \\ Cantabria. Santander. Spain.; ${ }^{(3)}$ Spanish Institute of Oceanography. \\ Oceanographic Center of Santander. Santander. Spain.
}

Introduction: Nowadays there is no doubt that aquaculture is one of the main sources of the world's food finfish (1).But this fast growth has some ecological drawbacks as a result of the high amounts of chemicals, including antibiotics, used in this activity. So it is essential to develop strategies to reduce the use of such antibiotics without decreasing the productivity of the aquatic farms. One of the most promising strategies is the administration of probiotics to finfish, that improve the health of the animals by enhancing the fish response toward disease (2).

Objectives: The aim of this study wasto characterise the probiotic potential of 9 lactic acid bacteria (LAB) strains isolated from healthy wild fish guts. To achieve this objective, the sensitivity of the strains to $\mathrm{pH}$ and bile salts was assayed.

Methods: All LAB strains were grown in MRS broth supplemented with $1,5 \% \mathrm{NaCl}$ at $30^{\circ} \mathrm{C}$. To assay $\mathrm{pH}$ sensitivity, medium was adjustedat $\mathrm{pH} 3.0,5.0$ and 9.0. Bile salt concentrations used were $0.1,0.5$ and $1.0 \%$. Bacterial growth was monitored by reading the OD at $620 \mathrm{~nm}$ during 24 hours in a MultiskanTMFC (Thermo Scientific).

Results: Only one of the LAB strains was able to grow at $\mathrm{pH} 3.0$ (L. actobacillus) and three were able to grow at $\mathrm{pH} 9.0$ (2 Enterococcus strains and L. actococcus lactis strain). All the strains tolerated $0,1 \%$ bile salt concentration, but only Lb plantarumstrainwas able tofaceall bile salt concentrations.

Conclusions: These results showed that Lactobacillus plantarum strain has a promising potential to be used as probiotic in finfish aquaculture.

References: (1) FAO. Rome, Italy 24-27 October 2017.

(2) Zorriehzahra MJ et al. Veterinary Quarterly 36(4): 228-241, 2016

Conflict of interest: The authors of this manuscript certify that they do not have any conflict of interest. 


\section{$309 / 62$ \\ REPLACEMENT OF COLISTIN AND NEOMYCIN BY MEDIUM CHAIN FATTY ACIDS IN POSTWEANED PIGLETS}

\author{
R. Miranda Hevia; M. Gómez-García; Ó. Mencía-Ares; P. Gómez de \\ Nova; A. Carvajal Urueña; P. Rubio Nistal.
}

Group Digesporc. Department Animal Health. Faculty of Veterinary. University of León. León. Spain.

Introduction: The antimicrobial resistance is a global health problem associated to the extensive use of antibiotics in human and veterinary medicine. In swine production, antibiotics are usually used for prophylactic and metaphylactic purposes, particularly to control enteric diseases in post-weaning period. However, there is a need to reduce its use and to search for alternatives.

Objectives: The aim was to evaluate changes in the microbiota associated to the replacement of colistin and neomycin, administered in the diet of post-weaned piglets by MCFA.

Methods: A total of 384 post-weaned piglets from a commercial swine farm were randomly distributed into three experimental diets ( 8 pens/group and 16 animals/pen); control diet was based on the current prescription of antibiotics used in the farm while experimental diets did not include colistin and neomycin and were supplemented with two MCFA commercial products (SALMOSTAT ENTERONOVA or ENTERONOVA PLUS). Two faecal samples per pen were collected on 1, 7 and 33 days post-weaning. The number of total bacteria, Bacteroides, Lactobacillus, Bifidobacteriaceae and Enterobacteriaceae was estimated using qPCR.

Results: In general, Bacteroides increased through the experiment while the number of Lactobacillus, Bifidobacteriaceae and Enterobacteriaceae decreased. No significant differences were demonstrated between the control group and the experimental group supplemented with ENTERONOVA PLUS. However, the piglets that received the diet supplemented with SALMOSTAT ENTERONOVA had higher concentrations of Bacteroides and Lactobacillus at the end of the study.

Conclusions: The replacement of colistin and neomycin by MCFA did not cause significant changes in the counts of some of the most relevant bacterial groups in the microbiota. Nevertheless, our results suggest that a diet supplemented with SALMOSTAT ENTERONOVA would be associated with higher counts of Bacteroides and Lactobacillus.

References: Isaacson R \& Kim HB (2012); Kim HB et al (2011); Pérez-Cobas AE et al (2013)

Conflict of interest: The authors declare no conflict of interest in this communication

\section{$309 / 83$ \\ DEVELOPMENT OF AN IMMUNOASSAY FOR THE DETECTION OF LACTOFERRIN IN SWINE FAECES}

\author{
${ }^{(1)}$ R. Miranda Hevia; (2)J. Parrón-Quesada; (2)L. Sánchez-Paniagua; \\ ${ }^{(1)}$ M. Gómez-García; ${ }^{(1)}$ A. Carvajal Urueña; ${ }^{(1)}$ P. Rubio Nistal. \\ ${ }^{(1)}$ Group Digesporc. Department Animal Health. Faculty of \\ Veterinary. University of León. León. Spain.; ${ }^{(2)}$ Department \\ Food Technology. Faculty of Veterinary. University of Zaragoza. \\ Zaragoza. Spain.
}

Introduction: Lactoferrin is a glycoprotein present in tissues and secretions of mammals. It is associated with inflammatory processes participating in several physiological functions. In human medicine it is used as a marker of intestinal inflammation but its use in veterinary medicine has been limited by the scarce availability or high price of the tests

Objectives: The aim was to develop an ELISA test for the detection and quantification of lactoferrin in swine faeces

Methods: Lactoferrin was purified from milk and colostrum from sows. Anti-lactoferrin hyperimmune polyclonal serum and monoclonal antibodies were obtained in rabbit and mouse, respectively. Different combinations were tested in a sandwich ELISA. Finally, using the developed ELISA, lactoferrin was detected in the faeces of healthy piglets during lactation and pigs affected of infectious digestive diseases.

Results: Five specific hybridomas were obtained and supernatants were tested together with the polyclonal serum. One of the monoclonal antibodies was selected, purified and labeled with peroxidase. Finally, the combination that provided best results was the coating of the plates with the polyclonal antibody, incubation with faecal samples and the peroxidase-labelled monoclonal antibody as conjugate.

Using this ELISA test, a decreasing concentration of lacfoferrin in the faeces of lactating piglets throughout lactation was demonstrated. The concentration of lactoferrin was high in piglets during the first days while colostrum intake. A second lactoferrin peak was detected after weaning.

Moreover, we demonstrated a high concentration of lactoferrin in faeces of pigs affected by swine dysentery. In contrast, lactoferrin levels remain low in other infections such as ileitis, colibacilosis or PED

Conclusions: The developed ELISA test allows the detection and quantification of lactoferrin in the swine faeces. Lactoferrin concentration is increased in some infectious enteric diseases of pigs such as swine dysentery

References: Yang et al. (2000); González-Chávez et al. (2009), Zhou et al. (2014).

Conflict of interest: Authors declare no conflict of interest. 


\section{Nutrition\& Metabolism}

Abrahamsson, T. ..........................................................................................4

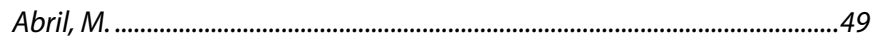

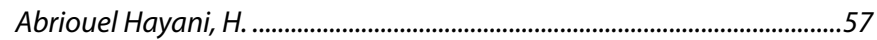

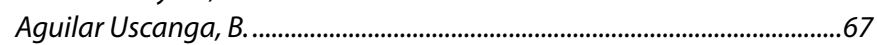

Alcántara Baena, C.....................................................................................................63

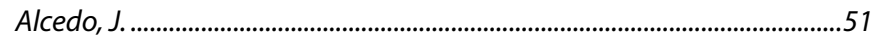

Alonso Fernández, S.....................................................................................

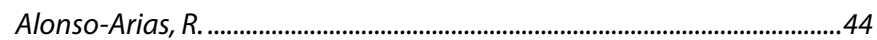

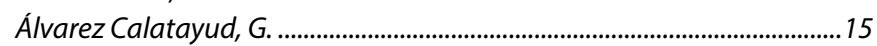

Alvarez Gonzalez, L. ........................................................................................... 70

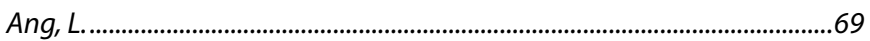

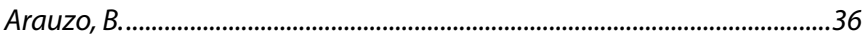

Arboleya Montes, S.................................................................................6

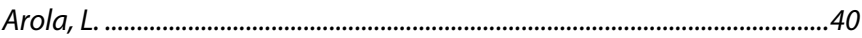

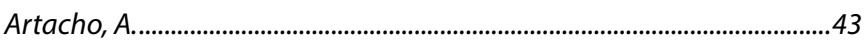

Astó Sanchez-Lafuente, E. ............................................................... 56, 68

Azagra Boronat, l.....................................................................................49

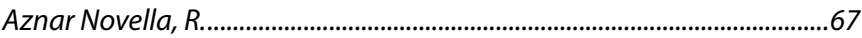

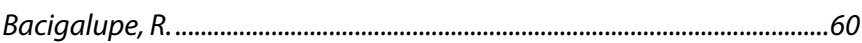

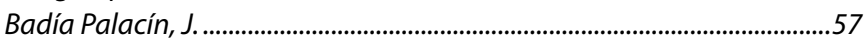

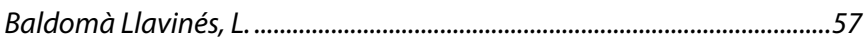

Barba Vidal, E............................................................................................

Bartolomé Suáldea, B........................................................................ 43, 62

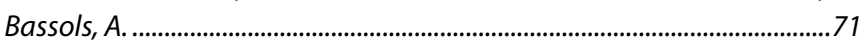

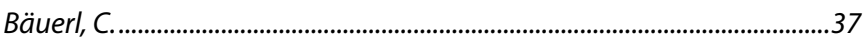

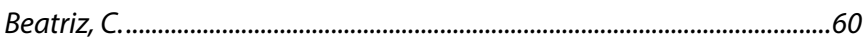

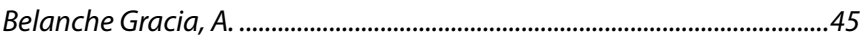

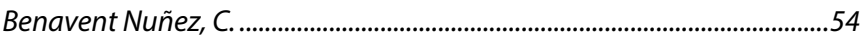

Benomar El Bakali ,N.............................................................................................5

Bittencourt Luciano, F......................................................................................6

Blanco-Míguez, A...................................................................................................4

Boix-Amorós, A............................................................................ 44, 60

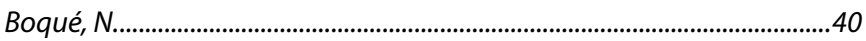

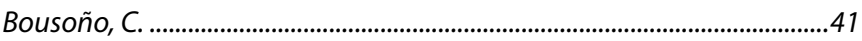

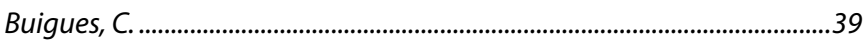

Busca Arenzana, K.....................................................................................6

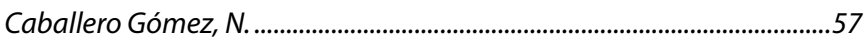

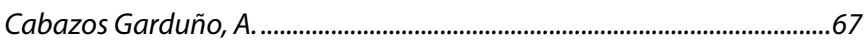

Cabello Olmo, M. ........................................................................................52

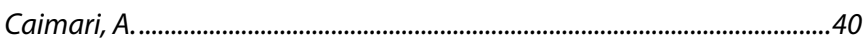

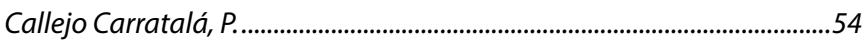

Campista Léon, S. ......................................................................................

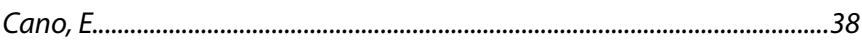

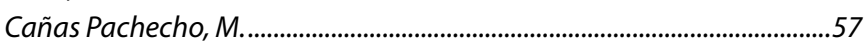

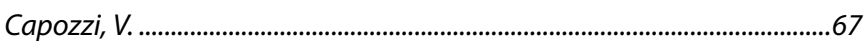

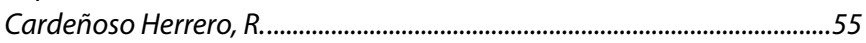

Carrion-Gutierrez, M...................................................................... 40, 56

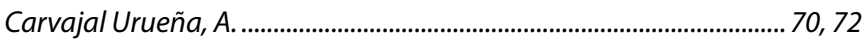

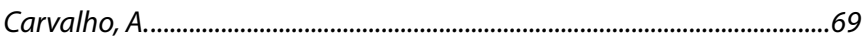

KARGER 9th Workshop SEPyP

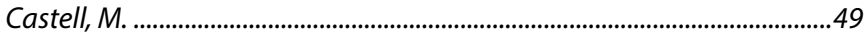

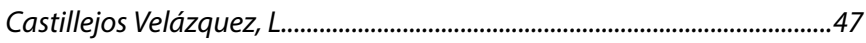

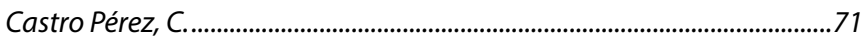

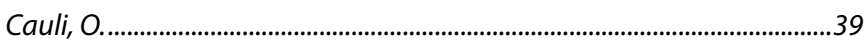

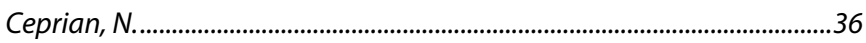

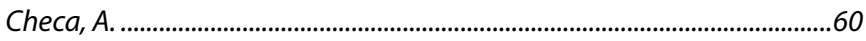

Chenoll-Cuadros, E.................................................................................40

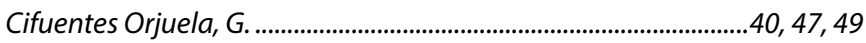

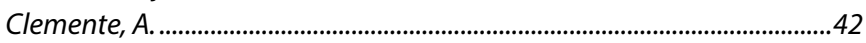

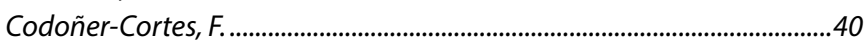

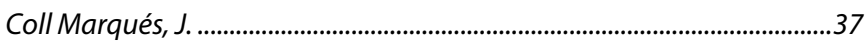

Collado Amores, M................................................................43, 44, 63, 65

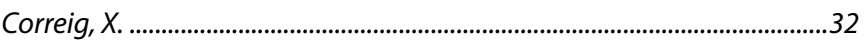

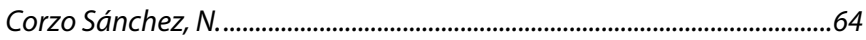

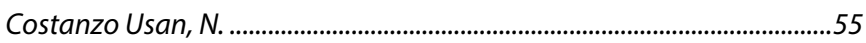

Costillas Alonso, S................................................................................... 70

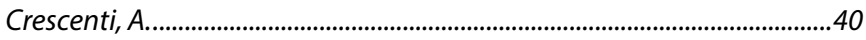

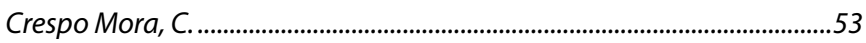

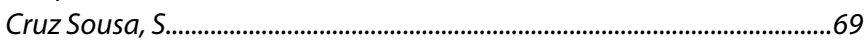

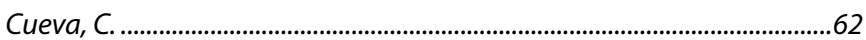

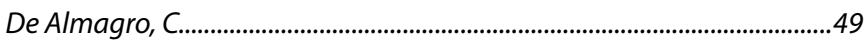

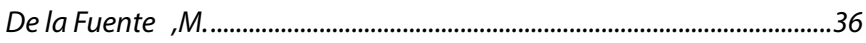

De La Visitación Pastor ,N...................................................35, 47, 48, 50

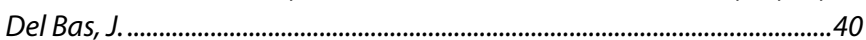

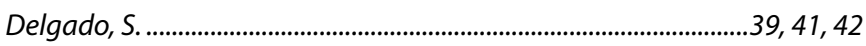

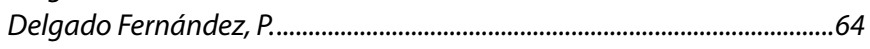

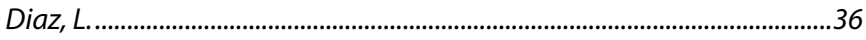

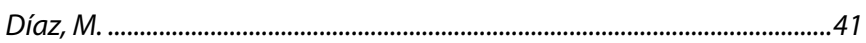

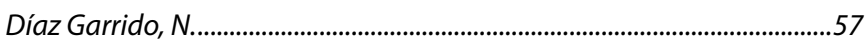

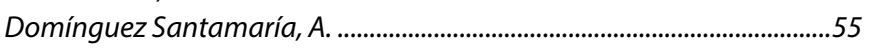

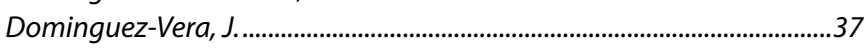

du Toit, E.........................................................................................4

Duarte Pérez, J.................................................................35, 47, 48, 50

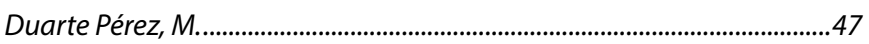

Dueñas Chasco, M............................................................................................5

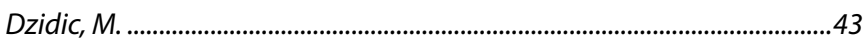

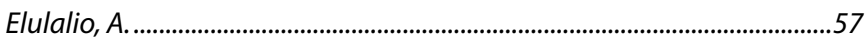

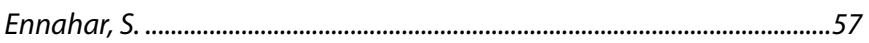

Espadaler Mazo, J............................................................................... 56, 68

Fábrega Fernández, M..................................................................................5

Fernández, J.................................................................................................4

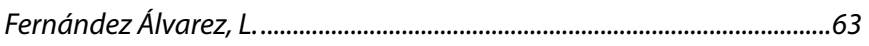

Fernández González, N. ..........................................................................61

Fernández Navarro, T.......................................................................35, 43, 48

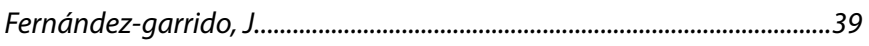

Fernández-Riverola, F.............................................................................4

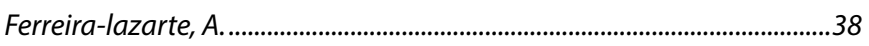

Ferrés Amat, E. .......................................................................................

Ann Nutr Metab 2018;72 (suppl 1): 1-76 


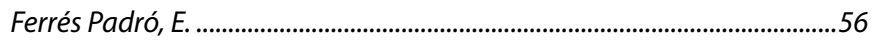

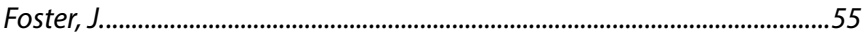

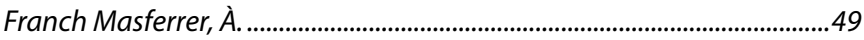

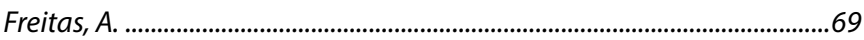

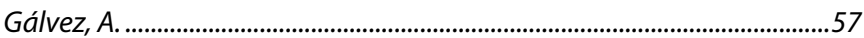

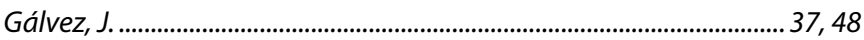

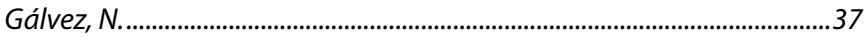

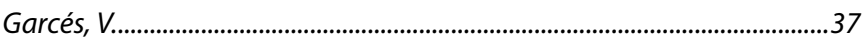

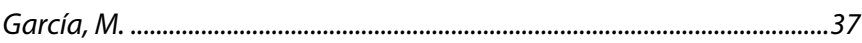

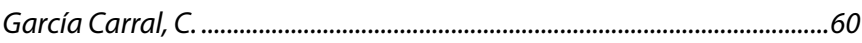

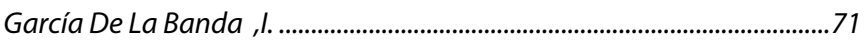

Garcia Diez, M.

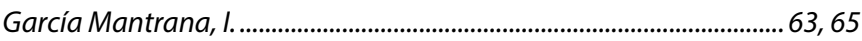

Garcia Morales, E. .............................................................................................6

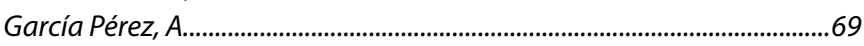

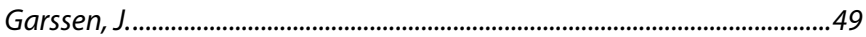

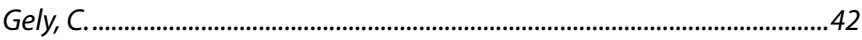

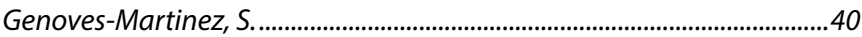

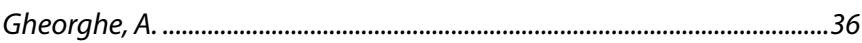

Gil Sánchez, I........................................................................................................62

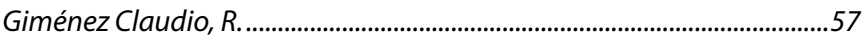

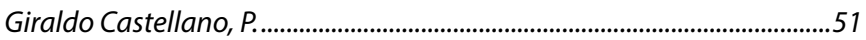

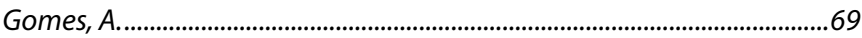

Gómez de Nova ,P..........................................................................................

Gómez Notario, C. ............................................................................................

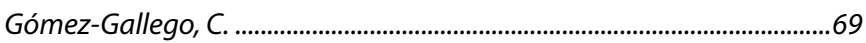

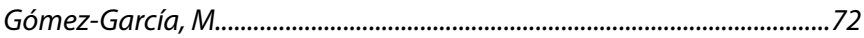

González, A. ..................................................................................................

González de los Reyes Gavilan ,C.................................................43, 48, 61

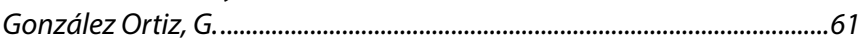

Gonzalez Reynoso, O. ....................................................................................67

González Solares, S. ........................................................................35, 43, 48

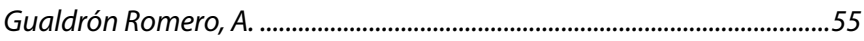

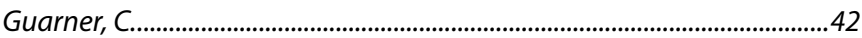

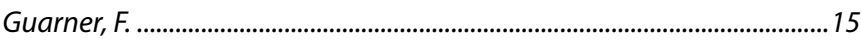

Gueimonde, M....................................................... 35, 41, 43, 48, 61, 69

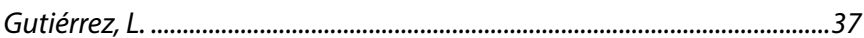

Gutíerrez Díaz, I.................................................................................... 43, 48

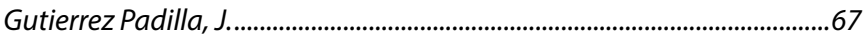

Hernández, P..............................................................................................................36

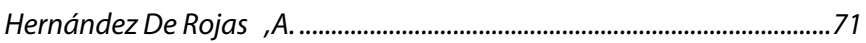

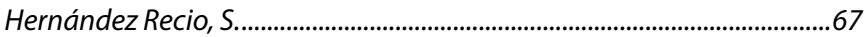

Hernández-hernández, O. ........................................................................64

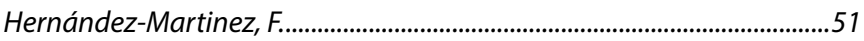

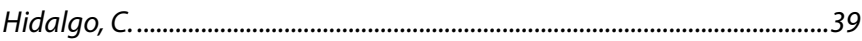

Hidalgo-Cantabrana, C. .................................................................................4

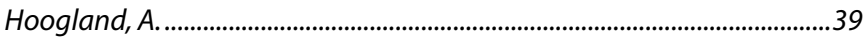

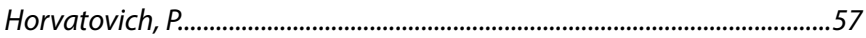

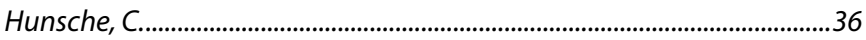

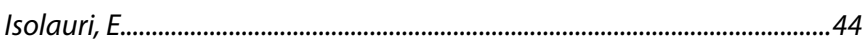

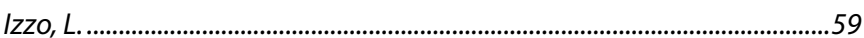

Jara Pérez, J..................................................................................................63

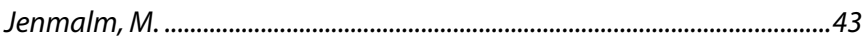

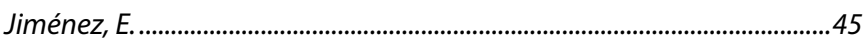

$74 \quad$ Ann Nutr Metab 2018;72 (suppl 1): 1-76

DOI: $10.11 .59 / 000487036$

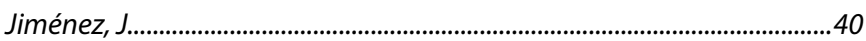

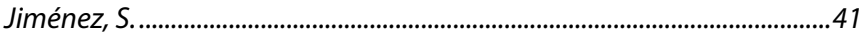

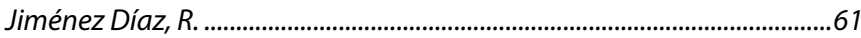

Jiménez Moleón, R...............................................................35, 47, 48, 50

Jiménez-Díaz, J. ........................................................................................ 51, 53

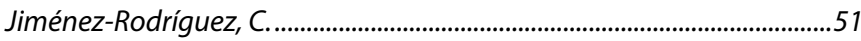

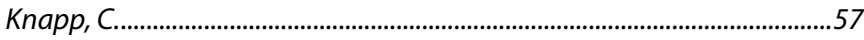

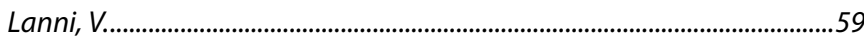

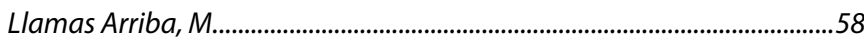

Llatas Mateu, A..............................................................................................6

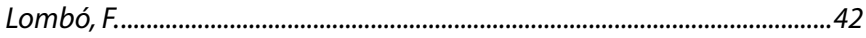

López, P......................................................................... 58, 67, 70, 71

López Colom, P.................................................................................... 47, 71

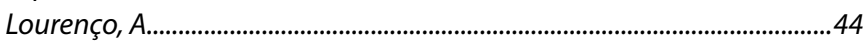

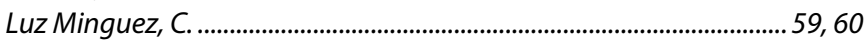

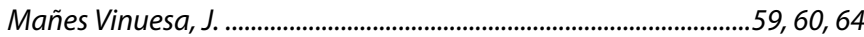

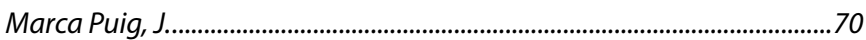

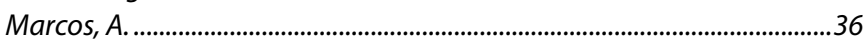

Mareque Bueno, J. ...........................................................................................

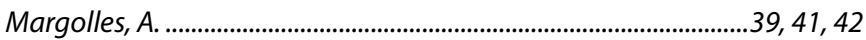

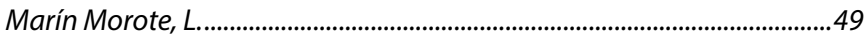

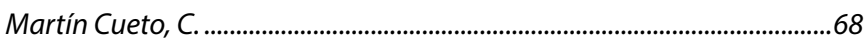

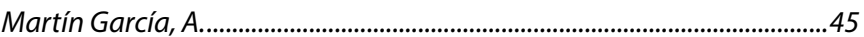

Martín Orúe, S. ........................................................................... 61, 62, 71

Martínez Álvarez, N.......................................................................................39

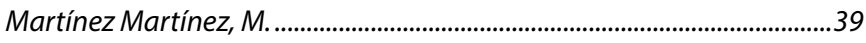

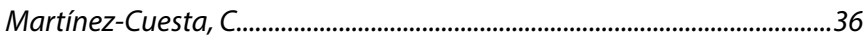

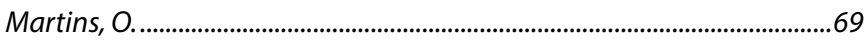

Massot-Cladera, M............................................................................................4

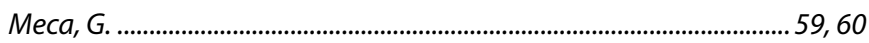

Medrano Engay, B..................................................................................

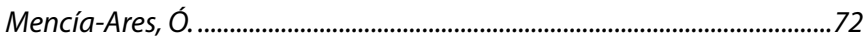

Mendez Sobrado, I.......................................................................................68

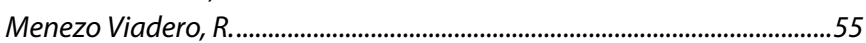

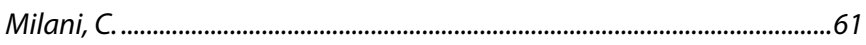

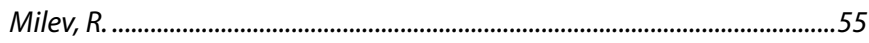

Mira, A. ............................................................................ 31, 43, 44, 60

Miranda Hevia, R. ....................................................................... 70, 72

Mohedano, M. ........................................................................................ 67,70

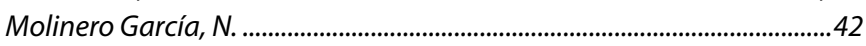

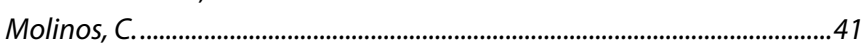

Monsalve Ríos, A...................................................................................6

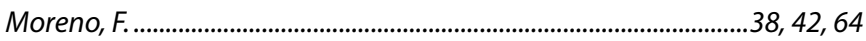

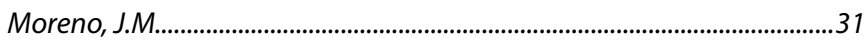

Moreno Andújar, F.................................................................................. 38, 64

Moreno Arribas, M........................................................................... 43, 62

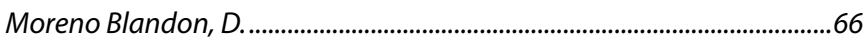

Moreno Muñoz, J........................................................................40, 47, 49

Moro-García, M................................................................................................... 44

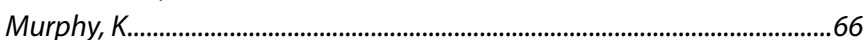

Navarro Tapia, E........................................................................... 56, 68

Navarro-López, V. ............................................................................. 40, 56

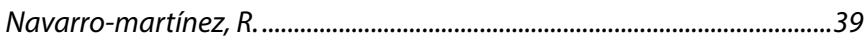

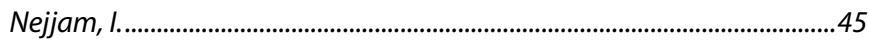

9th Workshop SEPyP 


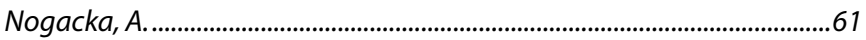

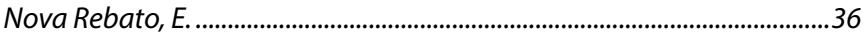

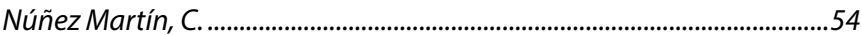

Olano, $A$

42,64

Olivares, $M$

.....37

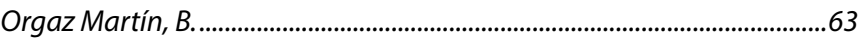

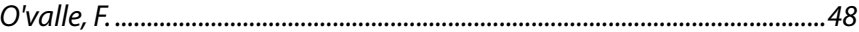

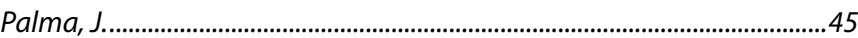

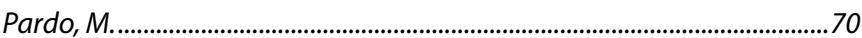

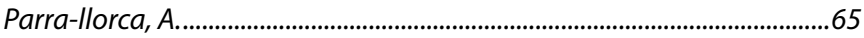

Parrón-Quesada, J.......................................................................................72

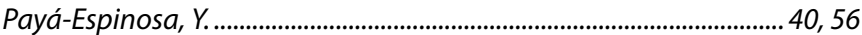

Peirotén, Á. ............................................................................................... 58

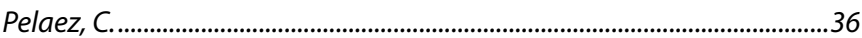

Pellejero, A.

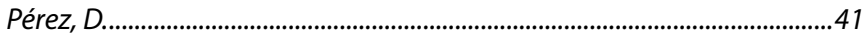

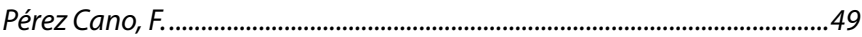

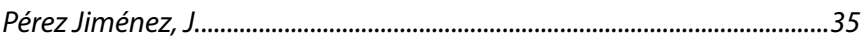

Pérez Martínez, G....................................................................................... 22, 37

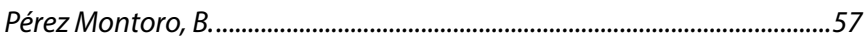

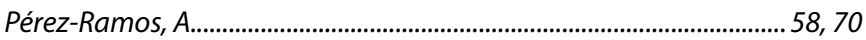

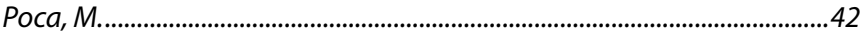

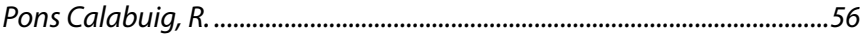

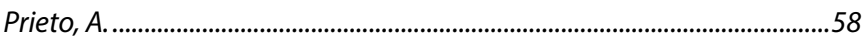

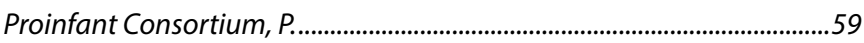

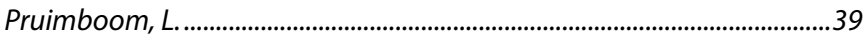

Puello Caballero, L........................................................................................6

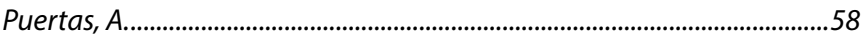

Quiles Beses, J...............................................................................................64

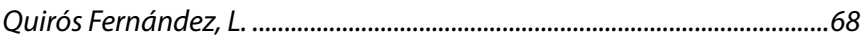

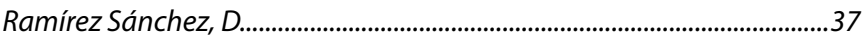

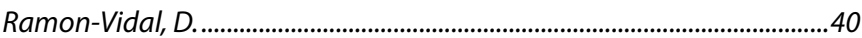

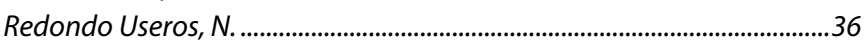

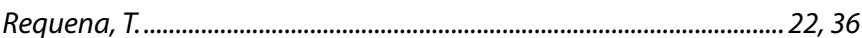

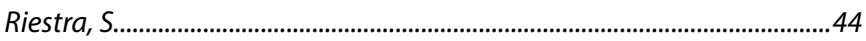

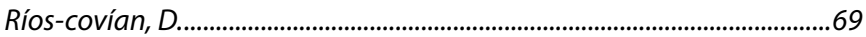

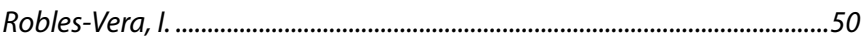

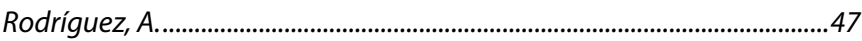

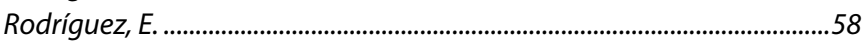

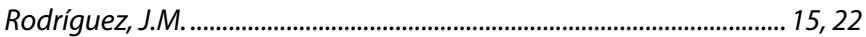

Rodriguez Arreola, A............................................................................67

Rodríguez Carrio, J. .......................................................................................48

Rodríguez Gómez, J.........................................................................41, 60, 63

Rodríguez Lagunas, M......................................................................................4

Rodríguez Palmero, M. ...................................................................... 40, 49

Rodríguez-de-Vera, B................................................................ 51, 53

Rodríguez-Nogales, A....................................................................37, 48

Romero-Pérez, M.................................................................. 47, 48, 50

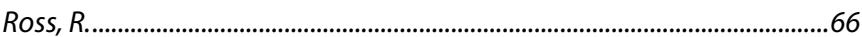

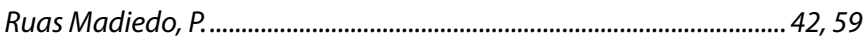

Rubio Nistal, $P$.................................................................................. 70, 72

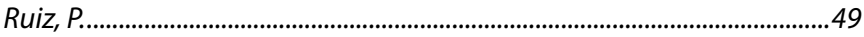

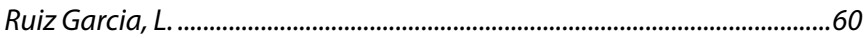

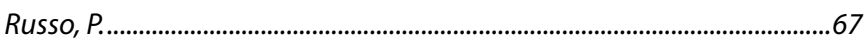

9th Workshop SEPyP

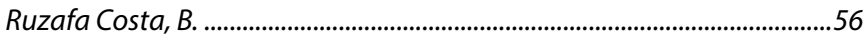

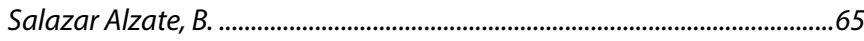

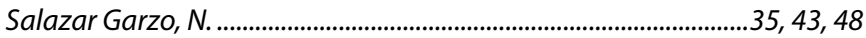

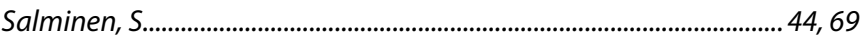

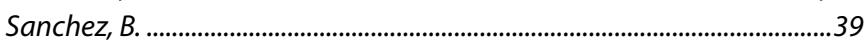

Sánchez, M........................................................................... 35, 47, 48, 50

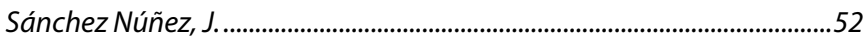

Sánchez Santos, M....................................................................35, 47, 50

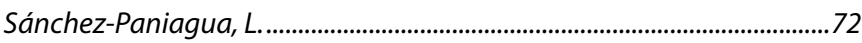

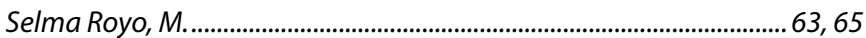

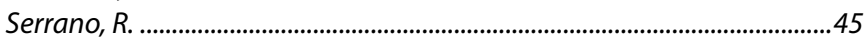

Serrano Niño, J ..........................................................................................67

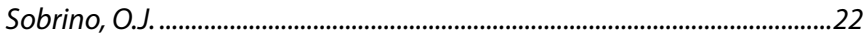

Solà Oriol, D. ................................................................................................. 71

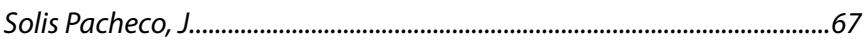

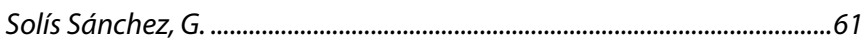

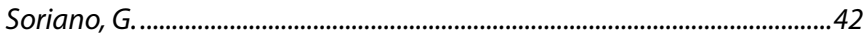

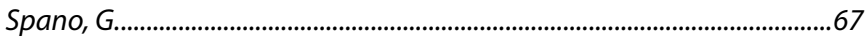

Stanton, C.

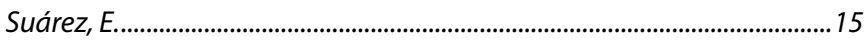

Suárez Díaz, A....................................................................................................4

Suárez Fernández, J................................................................................6

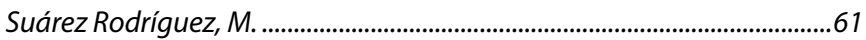

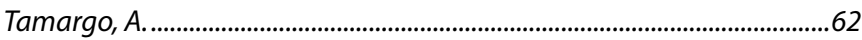

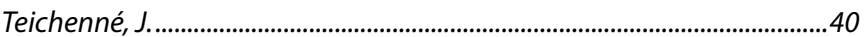

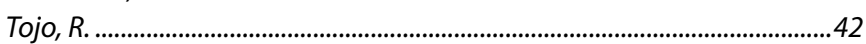

Toral Jiménez, M................................................................. 35, 47, 48, 50

Torrijos Caparrós, R. ....................................................................................64

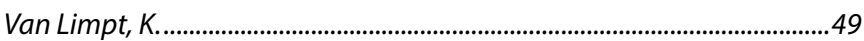

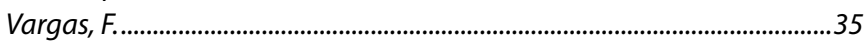

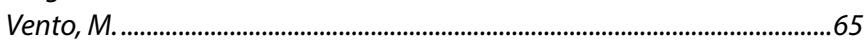

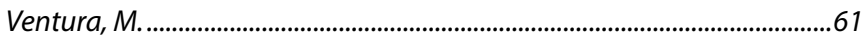

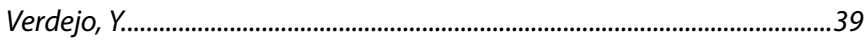

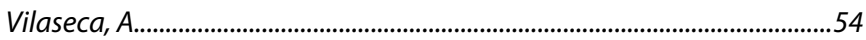

Villamiel Guerra, M................................................................................. 38, 64

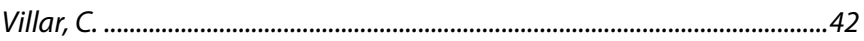

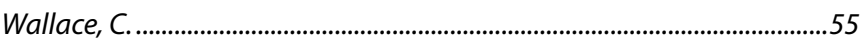

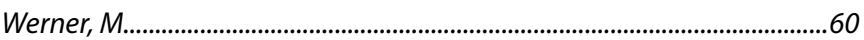

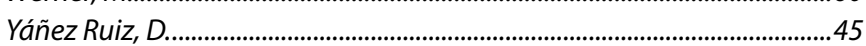

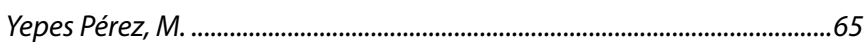

Yépez Latorre, A.......................................................................................67

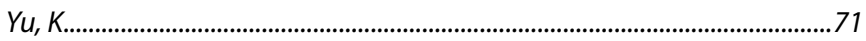

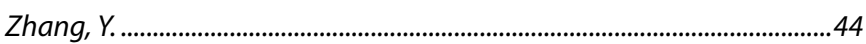

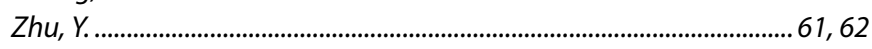

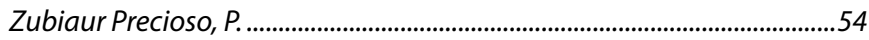

Ann Nutr Metab 2018;72 (suppl 1): 1-76 
